\title{
Allenes and Transition Metals: A Diverging Approach to Heterocycles
}

\author{
Kay M. Brummond and Branko Mitasev \\ Department of Chemistry, University of Pittsburgh, Pittsburgh, PA 15260
}

\section{Supporting Information}

\section{Part 1}

General methods. page $\mathrm{s} 2$ General procedure for the preparation of propargylic esters of $\mathrm{N}$ carbamate protected amino acids.............................page s3 General procedure for the preparation of $\mathrm{Cbz}-\mathrm{N}$-allenic amino acid methyl esters via a three step reaction sequence........................page s3 General procedure for $\mathrm{N}$-alkylation of $\mathrm{N}$-protected allenic amino acid

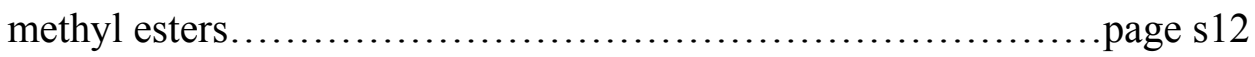
General procedure for the rhodium catalyzed formal Alder-ene

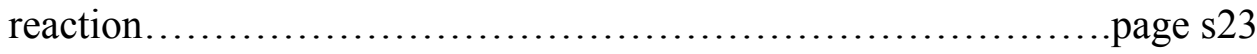
General procedure for rhodium catalyzed Pauson-Khand reaction for the

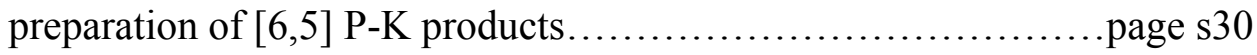
General procedure for the molybdenum mediated allenic Pauson-Khand reaction for the formation of $[5,5] \mathrm{P}-\mathrm{K}$ products..................page s38

\section{Part 2}

${ }^{1} \mathrm{H}$ NMR and ${ }^{13} \mathrm{C}$ NMR spectral data for all compounds............page s47 


\section{General Methods}

Unless otherwise noted, all reactions were carried out under nitrogen atmosphere. All commercially available compounds were purchased from Aldrich Chemical Co., GFS Chemicals, Strem Chemicals, Acros Organics and Advanced Chemtech and used as received, unless otherwise specified. Carbon monoxide gas (99.9\%) was purchased from Matheson Tri-Gas and used as received. $\mathrm{ZnCl}_{2}(0.5 \mathrm{M}$ in THF) was purchased from Aldrich Chemical Co. and stored over $4 \AA$ molecular sieves. Tetrahydrofuran (THF), diethyl ether $\left(\mathrm{Et}_{2} \mathrm{O}\right)$ and dichloromethane $\left(\mathrm{CH}_{2} \mathrm{Cl}_{2}\right)$ were purified with alumina using the Sol-Tek ST-002 solvent purification system. Toluene and diisopropylamine were freshly distilled from $\mathrm{CaH}_{2}$ prior to use. Purification of the compounds by flash chromatography was performed by using silica gel (32-63 $\mu \mathrm{m}$ particle size, $60 \AA$ pore size) purchased from SAI. ${ }^{1}$ TLC analyses were performed on EM Science Silica Gel $60 \mathrm{~F}_{254}$ plates (250 $\mu \mathrm{m}$ thickness). HPLC purification was performed on a Varian-Prostar 210 instrument using a Varian Microsorb Dynamax 100-5 Si column (5 $\mu \mathrm{m}$ packing, $250 \mathrm{~mm}$ x $10 \mathrm{~mm}$ ). All ${ }^{1} \mathrm{H}$ and ${ }^{13} \mathrm{C}$ spectra were obtained on Bruker Avance $300 \mathrm{MHz}$, and chemical shifts

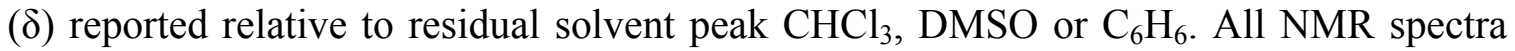
were obtained at room temperature unless otherwise specified. IR spectra were obtained using a Nicolet Avatar E.S.P. 360 FT-IR. EI mass spectrometry was performed on a Micromass Autospec high resolution mass spectrometer. Melting points were determined on a Mel-Temp instrument. The glassware was typically not oven-dried or flame-dried unless specified.

In some cases, mixtures of diastereomers were obtained and were not separated for characterization purposes. Consequently, the spectroscopic data has been reported as a mixture of diastereomers. Where possible resonances attributed to a single diastereomer are labeled accordingly: * denotes one diastereomer and ** denotes a second diastereomer. Where possible, the diastereomeric ratios were measured by integration of the resonances in the ${ }^{1} \mathrm{H}$ NMR.

\footnotetext{
${ }^{1}$ Still, W. C.; Kahn, M.; Mitra, A. J. Org. Chem. 1978, 43, 2923.
} 
General procedure for the preparation of propargylic esters of N-carbamate protected amino acids.

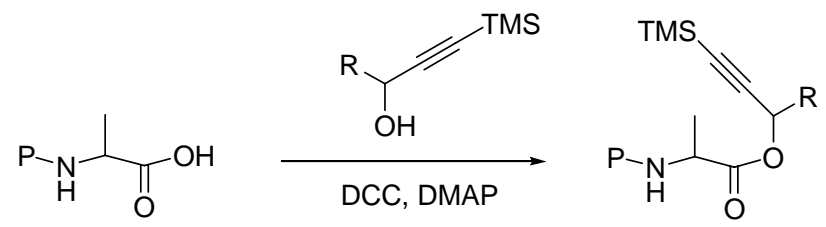

To a solution of propargylic alcohol (5 mmol, 1 equiv.), in $\mathrm{CH}_{2} \mathrm{Cl}_{2}(5-10 \mathrm{~mL})$, was added DMAP (0.5 mmol, 0.1 equiv.) and DCC ( $5.5 \mathrm{mmol}, 1.1$ equiv.) followed by N-protectedalanine ( $5.25 \mathrm{mmol}, 1.05$ equiv.) at room temperature under $\mathrm{N}_{2}$ atmosphere. Precipitation of a white solid was observed within $1 \mathrm{~min}$. The reaction mixture was stirred at room temperature for $1 \mathrm{~h}$, then filtered through a plug of silica gel eluting with hexanes-EtOAc, $1: 1, \mathrm{v} / \mathrm{v}$, and the clear liquid concentrated under vacuum. Purification by flash chromatography (hexanes-EtOAc, $4: 1, \mathrm{v} / \mathrm{v}$ ) afforded the desired ester.

General procedure for the preparation of N-Cbz-allenic amino acid methyl esters via a three step reaction sequence.

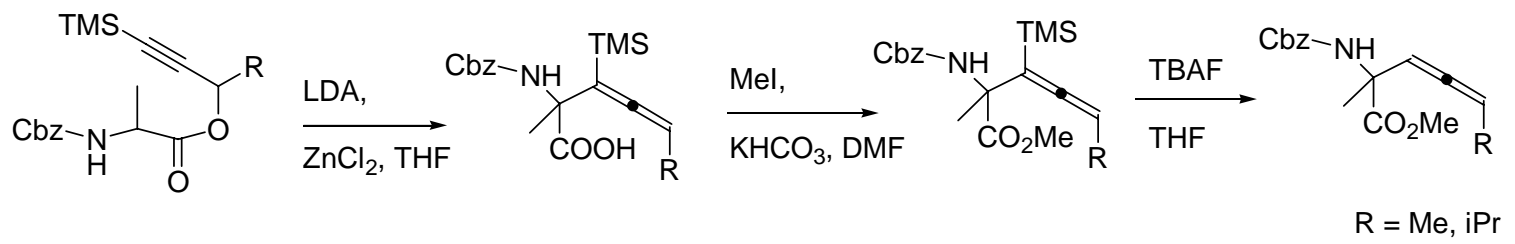

Preparation of LDA: To a flame dried $100 \mathrm{~mL}$ round bottomed flask, $i-\mathrm{Pr}_{2} \mathrm{NH}(10.0$ mmol) was added followed by THF $(10 \mathrm{~mL})$ under $\mathrm{N}_{2}$ atmosphere. The reaction flask was cooled to $-20{ }^{\circ} \mathrm{C}$ and n-butyllithium (10.0 mmol of $1.6 \mathrm{M}$ solution in hexanes) was added dropwise over $5 \mathrm{~min}$. The colorless solution was then cooled to $-78{ }^{\circ} \mathrm{C}$ and and held at that temperature for $30 \mathrm{~min}$.

Step 1. Ester-enolate Claisen rearrangement: The amino acid propargylic ester (4.0 mmol) was placed in a pear shaped flask as a solution in benzene and the solvent removed under high vacuum to azeotrope traces of water. The atmosphere was subsequently replaced with $\mathrm{N}_{2}$ and THF $(10 \mathrm{~mL})$ was added. The resulting solution was added via a syringe to the freshly prepared solution of LDA at $-78^{\circ} \mathrm{C}$. After $3-5 \mathrm{~min}$ of stirring, $\mathrm{ZnCl}_{2}\left(4.8 \mathrm{mmol}\right.$ of a $0.5 \mathrm{M}$ solution in THF) was added at $-78{ }^{\circ} \mathrm{C}$. The reaction 
mixture was allowed to warm to room temperature over $12 \mathrm{~h}$. Upon completion the reaction mixture was diluted with $\mathrm{Et}_{2} \mathrm{O}(200 \mathrm{~mL})$, and treated with $1 \mathrm{M} \mathrm{HCl}(200 \mathrm{~mL})$. The aqueous layer was extracted with $\mathrm{Et}_{2} \mathrm{O}(2 \times 100 \mathrm{~mL})$, and the organic layers were combined and washed with brine. Concentration under vacuum afforded a yellow oil.

Step 2. Methyl ester formation: The yellow oil from step 1 was dissolved in DMF (10 $\mathrm{mL})$. Pulverized $\mathrm{KHCO}_{3}(10 \mathrm{mmol})$ was added at room temperature, followed by MeI ( 8 mmol). The reaction mixture was stirred for 2-4 $\mathrm{h}$ until complete based upon TLC, and then diluted with water $(100 \mathrm{~mL})$. The cloudy mixture was extracted (benzene-EtOAc, 1 : $9, \mathrm{v} / \mathrm{v})(3 \times 75 \mathrm{~mL})$, the organic layers were combined, washed with brine $(2 \times 50 \mathrm{~mL})$ and concentrated under vacuum to afford a dark-yellow oil.

Step 3. TMS removal: The dark yellow oil from step 2 was dissolved in THF $(20 \mathrm{~mL})$, and phosphate buffer solution in water $(\mathrm{pH}=7,1 \mathrm{~mL})$ was added, followed by dropwise addition of TBAF ( $8 \mathrm{mmol}$ of a $1.0 \mathrm{M}$ solution in THF) at room temperature. The reaction mixture was stirred for $3 \mathrm{~h}$ at room temperature. Upon completion based upon TLC the reaction mixture was diluted with water $(100 \mathrm{~mL})$ and the aqueous layer extracted with $\mathrm{Et}_{2} \mathrm{O}(3 \times 100 \mathrm{~mL})$. The organic layers were combined, dried over $\mathrm{MgSO}_{4}$ and concentrated under vacuum. Purification of the crude residue by flash chromatography (hexanes-EtOAc, $4: 1$, v/v) afforded the title compound.

Reaction sequence for the preparation of 4 (Table 1, Entry a-d)

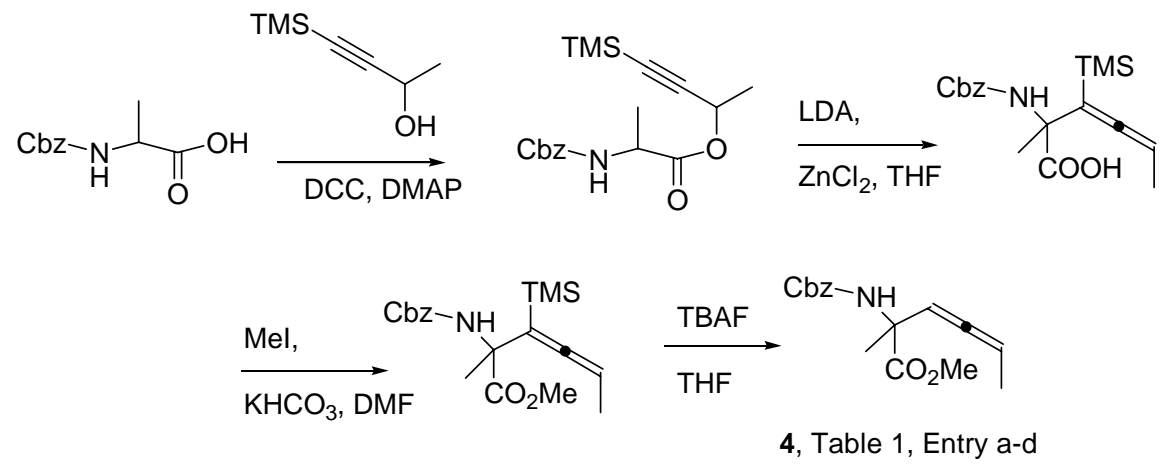




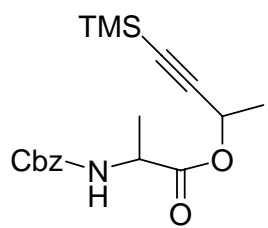

\section{2-Benzyloxycarbonylaminopropionic acid 1-methyl-3-trimethylsilylprop-2-ynyl}

ester. Prepared by the general procedure for the preparation of propargylic esters of Ncarbamate protected amino acids (page s3) from:

4-trimethylsilylbut-3-yn-2-ol (600 mg, $4.22 \mathrm{mmol}$ ), DMAP (51 mg, $0.42 \mathrm{mmol}), \mathrm{DCC}$ (956 mg, $4.64 \mathrm{mmol}$ ), $N$-Cbz-alanine (988 mg, $4.43 \mathrm{mmol}$ )

Yield (1.35 g, 92\%).

${ }^{1} \mathrm{H}$ NMR (300 MHz, $\mathrm{CDCl}_{3}$ ) $\delta$ 7.36-7.27 (m, 5H), 5.50 (quin, $J=6.8 \mathrm{~Hz}, 1 \mathrm{H}$ ), 5.33 (br s, 1H), $5.12(\mathrm{~s}, 2 \mathrm{H}), 4.41(\mathrm{sep}, 7.3 \mathrm{~Hz}, 1 \mathrm{H}), 1.52-1.41(\mathrm{~m}, 6 \mathrm{H}), 0.18(\mathrm{~s}, 9 \mathrm{H})^{*}, 0.17$ (s, $9 \mathrm{H}) * * ;{ }^{13} \mathrm{C} \mathrm{NMR}\left(75 \mathrm{MHz}, \mathrm{CDCl}_{3}\right) \delta 171.8,155.6,136.3,128.6,128.2,103.0,102.8$, $90.5,90.2,67.0,62.0,49.7,21.4,18.9,18.6,-0.2$; IR (thin film) v 3341, 2960, 2178, 1727, $1251 \mathrm{~cm}^{-1}$; MS m/z (\%) 348 (5), 270 (10), 178 (13), 134 (26), 91 (100); EI-HRMS calcd for $\mathrm{C}_{18} \mathrm{H}_{25} \mathrm{NO}_{4} \mathrm{Si} \mathrm{m} / \mathrm{z}\left[\mathrm{M}^{+}\right]$347.1553; found 347.1541.

* from diastereomer $1, * *$ from diastereomer 2.

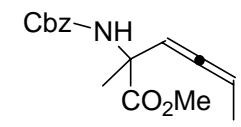

2-Benzyloxycarbonylamino-2-methylhexa-3,4-dienoic acid methyl ester. Prepared by the general procedure for the preparation of N-Cbz-allenic amino acid methyl esters via a three step reaction sequence (page s3):

Step 1: LDA (17 mmol), 2-benzyloxycarbonylaminopropionic acid 1-methyl-3trimethylsilylprop-2-ynyl ester $(2.36 \mathrm{~g}, 6.80 \mathrm{mmol}), \mathrm{ZnCl}_{2}(16.3 \mathrm{~mL}$ of a $0.5 \mathrm{M}$ solution in THF, $8.16 \mathrm{mmol})$; Step 2: $\mathrm{KHCO}_{3}(1.37 \mathrm{~g}, 13.6 \mathrm{mmol})$, MeI (0.67 mL, $\left.11 \mathrm{mmol}\right)$; Step 3: TBAF ( $6 \mathrm{~mL}$ of a 1.0 M solution in THF, $6 \mathrm{mmol})$.

Yield (970 mg, 49\%, 3 steps).

${ }^{1} \mathrm{H}$ NMR $\left(300 \mathrm{MHz}, \mathrm{CDCl}_{3}\right) \delta$ 7.36-7.32 (m, 5H), $5.50(\mathrm{bs}, 1 \mathrm{H}), 5.44-5.36(\mathrm{~m}, 2 \mathrm{H}), 5.13$ $(1 / 2 \mathrm{AB}, J=12.5 \mathrm{~Hz}, 1 \mathrm{H}), 5.08(1 / 2 \mathrm{AB}, J=12.3 \mathrm{~Hz}, 1 \mathrm{H}), 3.74$ (bs, 3H), 1.69-1.66 (m, $6 \mathrm{H}) ;{ }^{13} \mathrm{C} \mathrm{NMR}\left(75 \mathrm{MHz}, \mathrm{CDCl}_{3}\right) \delta 202.9,173.2,154.9,136.5,128.6,128.3,94.4,91.9$, 66.8, 58.6, 53.0, 23.6, 14.1; IR (thin film) v 3348, 2951, 1966, 1724, $1267 \mathrm{~cm}^{-1} . \mathrm{MS} \mathrm{m} / \mathrm{z}$ 
(\%) 289 (21), 274 (10), 230 (42), 91 (100); EI-HRMS calcd for $\mathrm{C}_{16} \mathrm{H}_{19} \mathrm{NO}_{4} \mathrm{~m} / \mathrm{z}\left[\mathrm{M}^{+}\right]$ 289.1314; found 289.1316 .

Reaction sequence for the preparation of 4 (Table 1, Entry e)
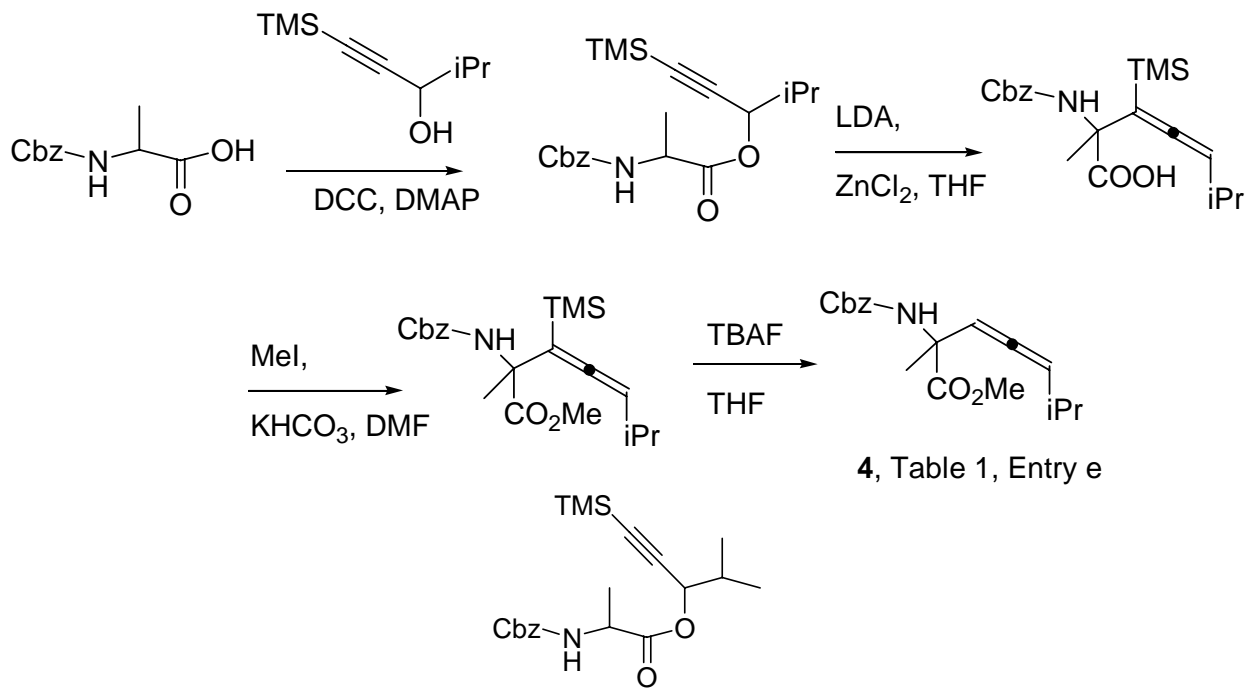

4, Table 1, Entry e

\section{2-Benzyloxycarbonylaminopropionic acid 1-isopropyl-3-trimethylsilylprop-2-ynyl}

ester. Prepared by the general procedure for the preparation of propargylic esters of Ncarbamate protected amino acids (page s3) from:

4-methyl-1-trimethylsilyl-pent-1-yn-3-ol (800 mg, $4.71 \mathrm{mmol}$ ), in DMAP (63 mg, 0.52 mmol), DCC (1.06 g, $5.15 \mathrm{mmol}), \mathrm{N}$-Cbz-alanine (1.15 g, $5.17 \mathrm{mmol})$.

Yield (1.65 g, 85\%).

${ }^{1} \mathrm{H}$ NMR $\left(300 \mathrm{MHz}, \mathrm{CDCl}_{3}\right) \delta$ 7.37-7.27 (m, 5H), $5.44(\mathrm{~m}, 1 \mathrm{H}), 5.29(\mathrm{~d}, \mathrm{~J}=5.6 \mathrm{~Hz}$, $1 \mathrm{H})^{*}, 5.25(\mathrm{~d}, J=5.7 \mathrm{~Hz}, 1 \mathrm{H})^{* *}, 5.12(\mathrm{~s}, 2 \mathrm{H}), 4.49-4.38(\mathrm{~m}, 1 \mathrm{H}), 2.05-1.94(\mathrm{~m}, 1 \mathrm{H})$, $1.44(\mathrm{~d}, J=7.1 \mathrm{~Hz}, 1 \mathrm{H}), 1.03-0.98(\mathrm{~m}, 3 \mathrm{H}), 0.18(\mathrm{~s}, 9 \mathrm{H}) *, 0.17(\mathrm{~s}, 9 \mathrm{H}) * * ;{ }^{* 3} \mathrm{C}$ NMR $(75$ $\left.\mathrm{MHz}, \mathrm{CDCl}_{3}\right) \delta 171.9,171.7,155.5,136.3,128.4,128.1,128.0,100.8,100.6,91.6,91.4$, $70.4,70.3,66.8,49.8,49.5,32.4,32.3,18.8,18.3,18.0,18.0,17.4$; IR (thin film) v 3340, 2965, 2180, 1728, $1526 \mathrm{~cm}^{-1}$; MS m/z (\%) 376 (6), 375 (14), 295 (25), 280 (34), 223 (50), 91 (100); EI-HRMS calcd for $\mathrm{C}_{20} \mathrm{H}_{29} \mathrm{NO}_{4} \mathrm{Si} \mathrm{m} / z$ [M $\left.\mathrm{M}^{+}\right] 375.1866$; found 375.1863.

diastereomer $1, * *$ diastereomer 2 


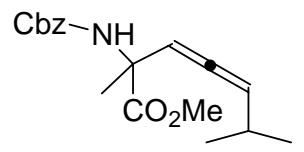

2-Benzyloxycarbonylamino-2,6-dimethylhepta-3,4-dienoic acid, methyl ester.

Prepared by following the general procedure the preparation of $\mathrm{N}-\mathrm{Cbz}$-allenic amino acid methyl esters via a three step reaction sequence (page s3):

Step 1: LDA (5.3 mmol), 2-benzyloxycarbonylaminopropionic acid 1-isopropyl-3trimethylsilylprop-2-ynyl ester (800 mg, $2.13 \mathrm{mmol}), \mathrm{ZnCl}_{2}(5.1 \mathrm{~mL}$ of a $0.5 \mathrm{M}$ solution in THF, $2.6 \mathrm{mmol})$; Step 2: $\mathrm{KHCO}_{3}(537 \mathrm{mg}, 5.32 \mathrm{mmol})$, MeI (265 $\left.\mu \mathrm{L}, 4.26 \mathrm{mmol}\right)$; Step 3: TBAF (2.1 mL of a 1.0 M solution in THF, $2.1 \mathrm{mmol})$.

Yield (325 mg, 48\%, 3 steps).

${ }^{1} \mathrm{H}$ NMR $\left(300 \mathrm{MHz}, \mathrm{CDCl}_{3}\right) \delta$ 7.38-7.32 (m, 5H), 5.51-5.42 (m, 2H), $5.13(1 / 2 \mathrm{AB}, J=$ $12.3 \mathrm{~Hz}, 1 \mathrm{H}), 5.07(1 / 2 \mathrm{AB}, J=12.2 \mathrm{~Hz}, 1 \mathrm{H}), 3.73$ (bs, 3H), 2.39-2.24 (m, 1H), 1.67 (s, $3 \mathrm{H}), 1.01(\mathrm{~d}, J=6.8 \mathrm{~Hz}, 3 \mathrm{H}), 1.01(\mathrm{~d}, J=6.8 \mathrm{~Hz}, 3 \mathrm{H}) ;{ }^{13} \mathrm{C} \mathrm{NMR}\left(75 \mathrm{MHz}, \mathrm{CDCl}_{3}\right) \delta$ 200.5, 173.0, 154.8, 136.4, 128.5, 128.1, 104.5, 96.0, 66.6, 58.4, 52.7, 27.9, 23.4, 22.2, 22.1; IR (thin film) v 3353, 2960, 1965, 1728, $1264 \mathrm{~cm}^{-1}$; MS m/z (\%) 318 (22), 317 (61), 302 (44), 274 (40), 258 (51), 91 (100); EI-HRMS calcd for $\mathrm{C}_{18} \mathrm{H}_{23} \mathrm{NO}_{4} \mathrm{~m} / \mathrm{z}\left[\mathrm{M}^{+}\right]$ 317.1627; found 317.1626 .

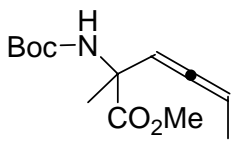

4, Table 1, Entry $n$

2-tert-Butoxycarbonylamino-2-methylhexa-3,4-dienoic acid, methyl ester. This compound has been previously synthesized in the Curran group via a modified Claisen rearrangement procedure. $^{2}$
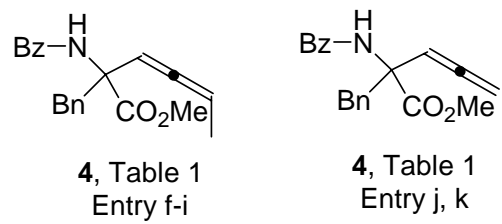

These compounds have been synthesized previously by Castelhano. ${ }^{3}$ Comparable yields were obtained. Compound 4 (Entry f-i), was obtained as a mixture of diastereomers in

\footnotetext{
${ }^{2}$ Curran, D. P.; Werner, S.; Unpublished results.
} 
ratio $1.7: 1$ resulting from the Claisen rearrangement. The diastereomeric ratio was retained in the subsequent alkylation, and rhodium catalyzed P-K reaction.

Reaction sequence for the preparation of 4 (Table 1, Entry 1, m)

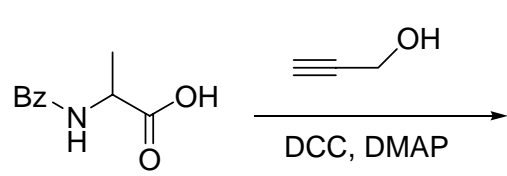<smiles>C#CCOC(=O)C(C)NC(=O)c1ccccc1</smiles>
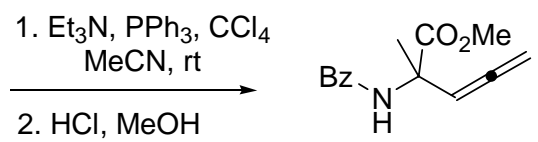

4, Table 1, Entry I,m<smiles>C#CCOC(=O)C(C)NC(=O)c1ccccc1</smiles>

2-Benzoylaminopropionic acid prop-2-ynyl ester. To a solution of propargyl alcohol (9.7 mL, $0.17 \mathrm{~mol})$ in $\mathrm{CH}_{2} \mathrm{Cl}_{2}(400 \mathrm{~mL})$ was added DMAP (100 mg, $\left.0.820 \mathrm{mmol}\right)$ and DCC $(22.9 \mathrm{~g}, 0.111 \mathrm{~mol})$ followed by $N$-benzoyl-alanine $(21.4 \mathrm{~g}, 0.111 \mathrm{~mol})$. A white precipitate forms immediately. After stirring for $36 \mathrm{~h}$ at room temperature the reaction mixture was filtered using a Büchner funnel to remove the solid. The resulting colorless solution was concentrated under vacuum and dissolved in EtOAc. Recrystallization (hexanes-EtOAc, $4: 1$, v/v) afforded the title compound (17.4 g, 68\%).

mp range 94.5-97.5 ${ }^{\circ} \mathrm{C} ;{ }^{1} \mathrm{H}$ NMR $\left(300 \mathrm{MHz}, \mathrm{CDCl}_{3}\right) \delta$ 7.78-7.76 (m, 2H), 7.75-7.35 (m, $3 \mathrm{H}$ ), 6.98 (br d, $J=6.9 \mathrm{~Hz}, 1 \mathrm{H}$ ), 4.79 (quin, $J=7.2 \mathrm{~Hz}, 1 \mathrm{H}$ ), 4.76 (dd, $J=15.5,2.5 \mathrm{~Hz}$, 1H), 4.69 (dd, $J=15.5,2.5 \mathrm{~Hz}, 1 \mathrm{H}), 2.51(\mathrm{t}, J=2.4 \mathrm{~Hz}, 1 \mathrm{H}), 1.50(\mathrm{~d}, J=7.2 \mathrm{~Hz}, 3 \mathrm{H})$; ${ }^{13} \mathrm{C}$ NMR $\left(75 \mathrm{MHz}, \mathrm{CDCl}_{3}\right) \delta 172.3,166.9,133.6,131.6,128.4,127.0,76.9,75.4,52.7$, 48.3, 18.0; IR (thin film) v 3294, 2940, 2128, 1749, 1642, 1536, $1164 \mathrm{~cm}^{-1}$; MS m/z (\%) 231 (43), 176 (20), 148 (63), 105 (100); EI-HRMS calcd for $\mathrm{C}_{13} \mathrm{H}_{13} \mathrm{NO}_{3} \mathrm{~m} / \mathrm{z}\left[\mathrm{M}^{+}\right]$ 231.0895; found 231.0899.

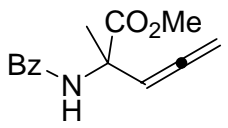

2-Benzoylamino-2-methylpenta-3,4-dienoic acid methyl ester. To a solution of 2-benzoylaminopropionic acid prop-2-ynyl ester $(13 \mathrm{mmol})$, in acetonitrile $(60 \mathrm{~mL})$, were added successively $\mathrm{Et}_{3} \mathrm{~N}(54.6 \mathrm{mmol}), \mathrm{CCl}_{4}(45.5 \mathrm{mmol}), \mathrm{PPh}_{3}(40.3 \mathrm{mmol})$ all at room temperature. After $3 \mathrm{~h}$ the starting material was consumed based upon TLC, and hexanes

\footnotetext{
${ }^{3}$ Castelhano, A. L.; Horne, S.; Taylor, G. J.; Tetrahedron 1988, 44, 5451.
} 
(80 $\mathrm{mL}$ ) was added. The precipitate was filtered (Büchner funnel) and the filtrate was concentrated under vacuum. The brown residue was then dissolved in methanol (100 mL) and a saturated solution of $\mathrm{HCl}$ in methanol $(3 \mathrm{~mL})$ was added. After stirring for $15 \mathrm{~min}$ the reaction mixture was poured into saturated aq. $\mathrm{NaHCO}_{3}(100 \mathrm{~mL})$, extracted with $\mathrm{Et}_{2} \mathrm{O}(5 \times 70 \mathrm{~mL})$, the organic layers were combined, dried over $\mathrm{MgSO}_{4}$ and concentrated under vacuum. Purification by flash chromatography (hexanes-EtOAc, $1: 1$, v/v) afforded the title compound (75-85\%) as a white solid.

mp range 135.1-136.6 ${ }^{\circ} \mathrm{C} ;{ }^{1} \mathrm{H}$ NMR $\left(300 \mathrm{MHz}, \mathrm{CDCl}_{3}\right) \delta$ 7.79-7.77 (m, 2H), 7.52-7.35 (m, 3H), 6.91 (br s, 1H), 5.64 (t, $J=6.6 \mathrm{~Hz}, 1 \mathrm{H}), 5.04$ (d, $J=6.6 \mathrm{~Hz}, 2 \mathrm{H}), 3.78(\mathrm{~s}, 3 \mathrm{H})$, $1.78(\mathrm{~s}, 3 \mathrm{H}) ;{ }^{13} \mathrm{C}$ NMR $\left(75 \mathrm{MHz}, \mathrm{CDCl}_{3}\right) \delta 206.6,172.8,166.4,134.2,131.6,128.5$, 126.9, 94.1, 80.2, 58.0, 52.9, 23.1; IR (thin film) v 3246, 2998, 1959, 1739, 1630, 1533; $\mathrm{cm}^{-1}$; MS m/z (\%) 245 (23), 230 (52), 186 (71), 105 (100); EI-HRMS calcd for $\mathrm{C}_{14} \mathrm{H}_{15} \mathrm{NO}_{3} \mathrm{~m} / \mathrm{z}\left[\mathrm{M}^{+}\right]$245.1052; found 245.1058.

Reaction sequence for the preparation of 4 (Table 1, Entry o).

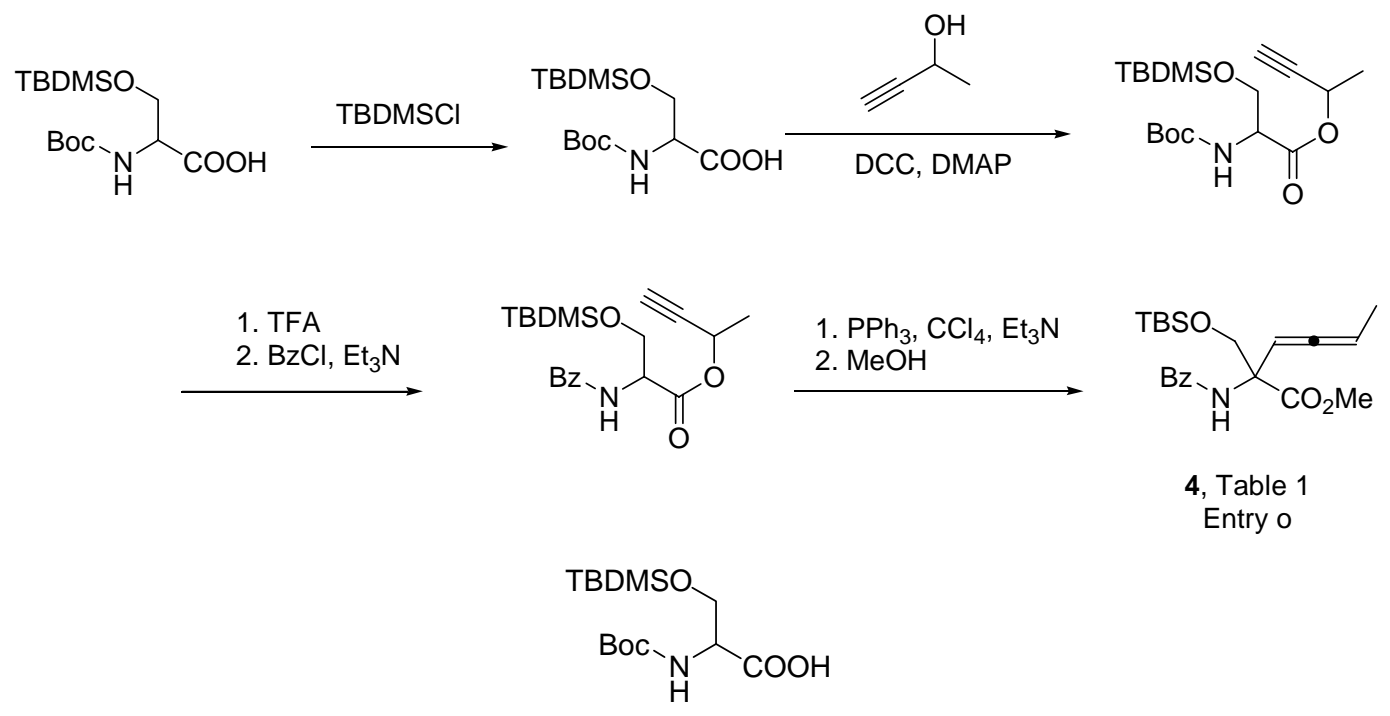

2-tert-Butoxycarbonylamino-3-(tert-butyldimethylsilyloxy)-propionic acid was prepared from $N$-Boc-serine according to a previously reported procedure. ${ }^{4}$

${ }^{4}$ Yoo, D.; Oh J. S.; Lee, D-W.; Kim, Y. G. J. Org. Chem., 2003, 68, 2979. 


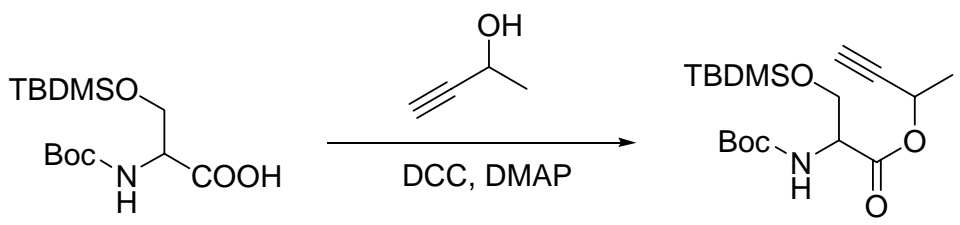

2-tert-Butoxycarbonylamino-3-(tert-butyldimethylsilyloxy)-propionic acid 1-methylprop-2-ynyl ester. Prepared by following the general procedure for the preparation of propargylic esters of $\mathrm{N}$-carbamate protected amino acids (page s3) from:

2-tert-Butoxycarbonylamino-3-(tert-butyldimethylsilyloxy)-propionic acid $(220 \mathrm{mg}$, $0.689 \mathrm{mmol}), 3$-butyne-2-ol (53 $\mu \mathrm{L}, 0.724 \mathrm{mmol}), \mathrm{DCC}$ (149 mg, $0.724 \mathrm{mmol})$, DMAP (8 mg, $0.007 \mathrm{mmol}$ ).

After flash chromatography (hexanes-EtOAc, $19: 1, \mathrm{v} / \mathrm{v})$, The title compound (187 mg, $73 \%$ ) was obtained as a $1: 1$ mixture of diastereomers.

${ }^{1} \mathrm{H}$ NMR $\left(300 \mathrm{MHz}, \mathrm{CDCl}_{3}\right) \delta$ 5.52-5.42 (m, 1H), 5.32 (br d, $\left.J=7.4 \mathrm{~Hz}, 1 \mathrm{H}\right), 4.36-4.30$ (m, 1H), 4.08-4.03 (m, 1H), $3.81(\mathrm{dd}, J=10.0,2.9 \mathrm{~Hz}, 1 \mathrm{H}), 2.45(\mathrm{~d}, J=2.1 \mathrm{~Hz}, 1 \mathrm{H}) *$, $2.41(\mathrm{~d}, J=2.1 \mathrm{~Hz}, 1 \mathrm{H})^{* *}, 1.50(\mathrm{~d}, J=6.7 \mathrm{~Hz}, 3 \mathrm{H})^{*}, 1.49(\mathrm{~d}, \mathrm{~J}=6.6 \mathrm{~Hz}, 3 \mathrm{H})^{* *}, 1.44(\mathrm{~s}$, $9 \mathrm{H}), 1.85(\mathrm{~s}, 9 \mathrm{H})^{* *}, 1.85(\mathrm{~s}, 9 \mathrm{H})^{*}, 0.03-0.01(\mathrm{~m}, 6 \mathrm{H}) ;{ }^{13} \mathrm{C} \mathrm{NMR}\left(75 \mathrm{MHz}, \mathrm{CDCl}_{3}\right) \delta$ 169.6, 155.4, 155.3, 81.6, 79.8, 73.3, 73.2, 63.6, 63.5, 61.1, 55.7, 55.6, 28.3, 25.7, 25.7, 21.3, 21.2, 18.1, -5.6; ; IR (thin film) v 3451, 3314, 2932, 2122, 1750, $1720 \mathrm{~cm}^{-1}$; MS m/z (\%) 372 (11), 316 (22), 258 (51), 206 (43), 57 (100); EI-HRMS calcd for $\mathrm{C}_{18} \mathrm{H}_{34} \mathrm{NO}_{5} \mathrm{Si}$ $\mathrm{m} / \mathrm{z}\left[\mathrm{M}+1^{+}\right]$372.2206; found 372.2201.

*from diastereomer 1; ** from diastereomer 2.
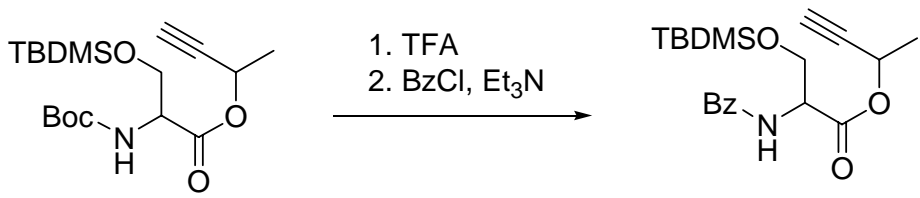

2-Benzoylamino-3-(tert-butyldimethylsilyloxy)-propionic acid 1-methylprop-2-ynyl ester. 2-tert-Butoxycarbonylamino-3-(tert-butyldimethylsilyloxy)-propionic acid 1methyl-prop-2-ynyl ester (1.48 g, $3.98 \mathrm{mmol})$ was dissolved in TFA (5 mL) and stirred for $5 \mathrm{~min}$ at room temperature, after which the excess TFA was removed under vacuum. The brown oil was purified by flash chromatography (hexanes-EtOAc, $3: 1$, v/v then EtOAc). After evaporation of the solvents the oil $(1.54 \mathrm{~g})$ was dissolved in $\mathrm{CHCl}_{3}(20$ $\mathrm{mL})$ and $\mathrm{Et}_{3} \mathrm{~N}(1.65 \mathrm{~mL}, 12.0 \mathrm{mmol})$ was added followed by benzoyl chloride $(444 \mu \mathrm{L}$, $3.84 \mathrm{mmol}$ ) at $0{ }^{\circ} \mathrm{C}$ under $\mathrm{N}_{2}$. The reaction mixture was stirred for $1 \mathrm{~h}$ after which it was 
diluted with $\mathrm{CHCl}_{3}(120 \mathrm{~mL})$. The organic phase was washed with acetic acid 5\% (50 $\mathrm{mL}$ ) and then sat. $\mathrm{NaHCO}_{3}(50 \mathrm{~mL})$, dried over $\mathrm{MgSO}_{4}$ and concentrated under vacuum. Purification by flash chromatography (gradient elution, hexanes-EtOAc, 19:1 to $4: 1$, $\mathrm{v} / \mathrm{v}$ ) afforded the title compound ( $900 \mathrm{mg}, 60 \%, 2$ steps).

${ }^{1} \mathrm{H}$ NMR $\left(300 \mathrm{MHz}, \mathrm{CDCl}_{3}\right) \delta$ 7.84-7.81 (m, 2H), 7.62-7.44 (m, 3H), $6.98(\mathrm{~d}, J=7.5 \mathrm{~Hz}$, $1 \mathrm{H})$, 5.61-5.44 (m, 1H), 4.97-4.86 (m, 1H), 4.23-4.17 (m, 1H), 4.00-3.96 (m, 1H), 2.49 $(\mathrm{d}, J=2.1 \mathrm{~Hz}, 1 \mathrm{H})^{*}, 2.46(\mathrm{~d}, J=2.1 \mathrm{~Hz}, 1 \mathrm{H})^{* *}, 1.57(\mathrm{~d}, J=5.7 \mathrm{~Hz}, 3 \mathrm{H})^{*}, 1.55(\mathrm{~d}, J=$ $5.7 \mathrm{~Hz}, 3 \mathrm{H})^{* *}, 0.89(\mathrm{~s}, 9 \mathrm{H})^{*}, 0.88(\mathrm{~s}, 9 \mathrm{H})^{* *}, 0.07(\mathrm{~s}, 3 \mathrm{H})^{*}, 0.06(\mathrm{~s}, 3 \mathrm{H})^{*}, 0.04(\mathrm{~s}, 3 \mathrm{H})^{* *}$, $0.04(\mathrm{~s}, 3 \mathrm{H})^{* *}$;

* from diastereomer 1

** from diastereomer 2

${ }^{13} \mathrm{C}$ NMR $\left(75 \mathrm{MHz}, \mathrm{CDCl}_{3}\right) \delta 169.5,169.4,167.0,166.9,134.0,131.8,128.7,127.0$, 81.5, 81.4, 73.6, 73.5, 63.5, 63.4, 61.5, 61.4, 54.7, 54.5, 25.8, 25.7, 21.3, 21.2, 18.2, 18.1, -5.5, -5.6; IR (thin film) v 3442, 3310, 2930, 2121, 1747, 1665, $1109 \mathrm{~cm}^{-1}$; MS m/z (\%) 360 (42), 318 (35), 105 (100); EI HRMS calcd for $\mathrm{C}_{20} \mathrm{H}_{29} \mathrm{NO}_{4} \mathrm{Si} \mathrm{m} / \mathrm{z}\left[\mathrm{M}^{+}\right]$375.1866, found 375.1852 .

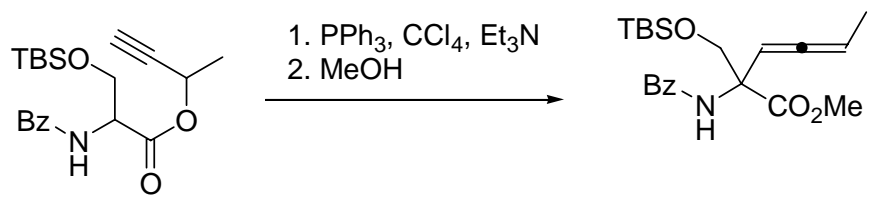

\section{2-Benzoylamino-2-(tert-butyldimethylsilyloxymethyl)-hexa-3,4-dienoic acid methyl} ester. To a solution of 2-Benzoylamino-3-(tert-butyldimethylsilyloxy)-propionic acid, 1methylprop-2-ynyl ester $(730 \mathrm{mg}, 1.95 \mathrm{mmol})$ in $\mathrm{MeCN}(10 \mathrm{~mL})$, were added in consecutive order $\mathrm{Et}_{3} \mathrm{~N}(758 \mu \mathrm{L}, 5.45 \mathrm{mmol}), \mathrm{CCl}_{4}(430 \mu \mathrm{L}, 4.49 \mathrm{mmol})$ and $\mathrm{PPh}_{3}(1.12$ $\mathrm{g}, 4.29 \mathrm{mmol})$ at room temperature. After $3 \mathrm{~h}$ at room temperature, the solvent was evaporated under vacuum and $\mathrm{MeOH}(10 \mathrm{~mL})$ was added followed by $\mathrm{Et}_{3} \mathrm{~N}(500 \mu \mathrm{L})$. After $3 \mathrm{~h}$ the solvents were evaporated and the crude mixture diluted with EtOAc (50 $\mathrm{mL})$ and then hexanes $(20 \mathrm{~mL})$. The formed precipitate was removed by filtration through a plug of silica gel. After removal of the solvents under vacuum the residue was purified by flash chromatography (hexanes-EtOAc, $9: 1, \mathrm{v} / \mathrm{v}$ ) to afford the title compound (660 $\mathrm{mg}, 87 \%)$. 
${ }^{1} \mathrm{H}$ NMR (300 MHz, $\left.\mathrm{CDCl}_{3}\right) \delta$ 7.80-7.77 (m, 2H), 7.57-7.41 (m, 3H), 7.12 (br s, 1H), $5.54(\operatorname{sex}, J=3.2 \mathrm{~Hz}, 1 \mathrm{H}) *, 5.44(\mathrm{sex}, J=3.1 \mathrm{~Hz}, 1 \mathrm{H}) * *, 5.38-5.30(\mathrm{~m}, 1 \mathrm{H}), 4.40(\mathrm{~d}, J=$ $9.8 \mathrm{~Hz}, 1 \mathrm{H})^{* *}, 4.31(\mathrm{~d}, J=9.8 \mathrm{~Hz}, 1 \mathrm{H})^{*}, 4.09$ (d, $\left.J=9.8 \mathrm{~Hz}, 1 \mathrm{H}\right), 3.80(\mathrm{~s}, 3 \mathrm{H}) * *, 3.80$ $(\mathrm{s}, 3 \mathrm{H})^{*}, 1.68-1.64(\mathrm{~m}, 3 \mathrm{H}), 0.84(\mathrm{~s}, 9 \mathrm{H}), 0.02(\mathrm{~s}, 3 \mathrm{H})^{* *}, 0.01(\mathrm{~s}, 3 \mathrm{H})^{*}, 0.00(\mathrm{~s}, 3 \mathrm{H})^{*},-$ $0.00(\mathrm{~s}, 3 \mathrm{H})^{* *}$

* major diastereomer; $* *$ minor diastereomer.

${ }^{13} \mathrm{C}$ NMR $\left(75 \mathrm{MHz}, \mathrm{CDCl}_{3}\right) \delta$ 204.0, 204.0, 171.1, 171.6, 166.3, 166.1, 134.8, 134.7, $131.3,128.4,126.8,126.7,90.7,90.4,89.9,89.7,64.6,64.4,64.1,52.8,52.7,25.5,17.9$, 13.8, 13.8, -5.6, -5.7; IR (thin film) v 3421, 3298, 2952, 1967, 1741, 1669, $1247 \mathrm{~cm}^{-1}$; MS m/z (\%) 374 (10), 332 (92), 244 (90), 105 (100); EI HRMS calcd for $\mathrm{C}_{21} \mathrm{H}_{31} \mathrm{NO}_{4} \mathrm{Si}$ $m / z\left[\mathrm{M}^{+}\right]$389.2022, found 389.2020.

General procedure for $\mathbf{N}$-alkylation of $\mathrm{N}$-protected allenic amino acid methyl esters.

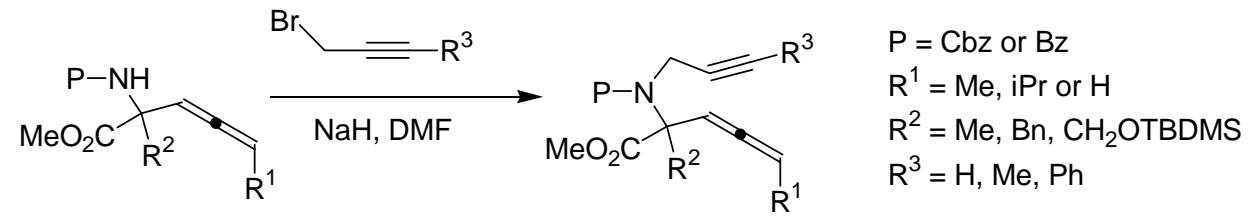

To a solution of the $\mathrm{N}$-protected amino acid $(1.0 \mathrm{mmol})$ in DMF $(4 \mathrm{~mL})$, was added $\mathrm{NaH}$ ( $2.0 \mathrm{mmol}$ of a $60 \%$ dispersion in mineral oil) at $0{ }^{\circ} \mathrm{C}$ under $\mathrm{N}_{2}$ atmosphere. After $2 \mathrm{~min}$ the alkyl bromide $(1.5-2.0 \mathrm{mmol})$ was added dropwise. The reaction mixture was stirred at room temperature for 15 - 45 min and upon completion as observed by TLC was quenched by cautiously pouring into water $(50 \mathrm{~mL})$. The mixture was extracted with EtOAc $(3 \times 50 \mathrm{~mL})$, the organic layers were combined, washed with brine, dried with $\mathrm{MgSO}_{4}$, and concentrated under vacuum. Purification by flash chromatography (hexanesEtOAc, $9: 1, \mathrm{v} / \mathrm{v}$ ) afforded the desired alkylated products.
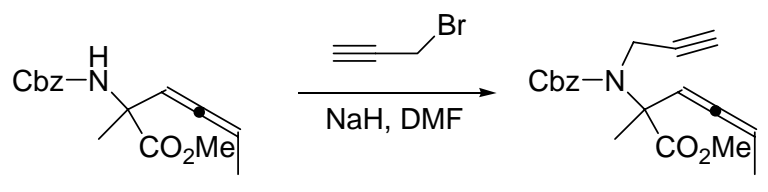

6, Table 1, Entry a

\section{2-(Benzyloxycarbonylprop-2-ynylamino)-2-methylhexa-3,4-dienoic acid methyl ester}

(6, Table 1, Entry a). Followed general procedure for N-alkylation of N-protected allenic amino acid methyl esters (page s12): 
2-Benzyloxycarbonylamino-2-methylhexa-3,4-dienoic acid methyl ester (240 mg, 0.83 $\mathrm{mmol}), \mathrm{NaH}$ (60\% dispersion in mineral oil, $70 \mathrm{mg}, 1.8 \mathrm{mmol})$, propargyl bromide (80\% wt. in toluene, $137 \mu \mathrm{L}, 1.25 \mathrm{mmol}$ ).

Purified by flash chromatography (hexanes-EtOAc, $9: 1, \mathrm{v} / \mathrm{v}$ ) to afford the title compound (210 mg, 77\%).

${ }^{1} \mathrm{H}$ NMR $\left(300 \mathrm{MHz}, \mathrm{C}_{6} \mathrm{D}_{6}, 323 \mathrm{~K}\right) \delta$ 7.22-7.01 (m, 5H), $5.63(\mathrm{sex}, J=3.1 \mathrm{~Hz}, 1 \mathrm{H}), 5.13-$ $5.04(\mathrm{~m}, 1 \mathrm{H}), 5.04(\mathrm{~s}, 2 \mathrm{H}), 4.21(\mathrm{~d}, J=18.1 \mathrm{~Hz}, 1 \mathrm{H}), 4.04(\mathrm{dd}, J=18.1,2.0 \mathrm{~Hz}, 1 \mathrm{H})$, 3.38 (br s, $3 \mathrm{H}), 1.88$ (t, $J=2.4 \mathrm{~Hz}, 1 \mathrm{H}), 1.77(\mathrm{~s}, 3 \mathrm{H}), 1.43(\mathrm{dd}, J=7.1,3.2 \mathrm{~Hz}, 1 \mathrm{H}) ;{ }^{13} \mathrm{C}$ NMR $\left(75 \mathrm{MHz}, \mathrm{C}_{6} \mathrm{D}_{6}, 323 \mathrm{~K}\right) \delta 205.5,172.2,155.3,137.0,93.8,90.6,81.3,71.5,67.8$, 64.9, 52.1, 34.2, 21.3, 13.6; IR (thin film) v 3288, 2950, 2115, 1966, 1745, 1704, 1254 $\mathrm{cm}^{-1}$; MS m/z (\%) 312 (13), 268 (34), 91 (100); EI-HRMS calcd for $\mathrm{C}_{18} \mathrm{H}_{18} \mathrm{NO}_{4} \mathrm{~m} / \mathrm{z}$ [M$15^{+}$] 312.1236; found 312.1243 .

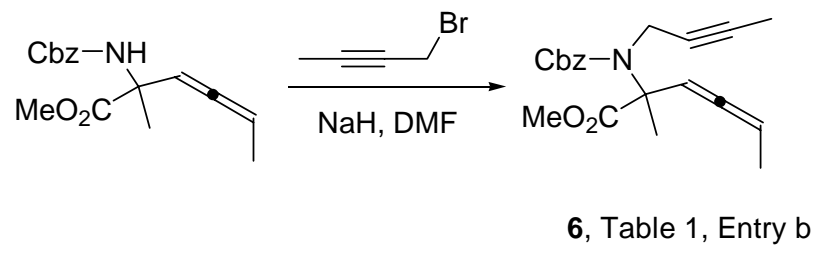

2-(Benzyloxycarbonyl-but-2-ynyl-amino)-2-methylhexa-3,4-dienoic acid, methyl ester (6, Table 1, Entry b). Followed general procedure for N-alkylation of N-protected allenic amino acid methyl esters (page s12):

2-Benzyloxycarbonylamino-2-methylhexa-3,4-dienoic acid, methyl ester (400 mg, 1.38 $\mathrm{mmol}$ ), $\mathrm{NaH}$ (110 $\mathrm{mg}$ of $60 \%$ dispersion in mineral oil, $2.76 \mathrm{mmol}$ ), 1-bromo-2-butyne (157 $\mu \mathrm{L}, 1.79 \mathrm{mmol})$.

Purification by flash chromatography (hexanes-EtOAc, $9: 1, \mathrm{v} / \mathrm{v}$ ) afforded the title compound (390 mg, 84\%).

${ }^{1} \mathrm{H}$ NMR (300 MHz, DMSO-d 6 , $\left.323 \mathrm{~K}\right) \delta$ 7.40-7.31 (m, 5H), 5.49-5.43 (m, 1H), $5.10 \mathrm{~s}$, 2H), $4.22(\mathrm{dq}, J=18.3,2.3 \mathrm{~Hz}, 1 \mathrm{H}), 4.11$ (dq, $J=18.2,2.2 \mathrm{~Hz}, 1 \mathrm{H}), 3.50$ (br s, 3H), 1.78 $(\mathrm{d}, J=2.0 \mathrm{~Hz}, 3 \mathrm{H}), 1.66(\mathrm{dd}, J=6.6,3.4 \mathrm{~Hz}, 3 \mathrm{H}), 1.54(\mathrm{~s}, 3 \mathrm{H}) ;{ }^{13} \mathrm{C} \mathrm{NMR}(75 \mathrm{MHz}$, DMSO-d 6,323 K) $\delta 204.0,171.5,154.3,136.1,127.9,127.5,127.2,92.5,89.9,78.5$, 75.9, 66.5, 63.4, 51.6, 33.6, 20.5, 13.0, 2.6; IR (thin film) v 2917, 1965, 1745, 1702, 1250 $\mathrm{cm}^{-1}$; MS m/z (\%) 341 (5), 326 (7), 282 (15), 91 (100); EI-HRMS calcd for $\mathrm{C}_{20} \mathrm{H}_{23} \mathrm{NO}_{4}$ $\mathrm{m} / \mathrm{z}\left[\mathrm{M}^{+}\right]$341.1627; found 341.1630. 


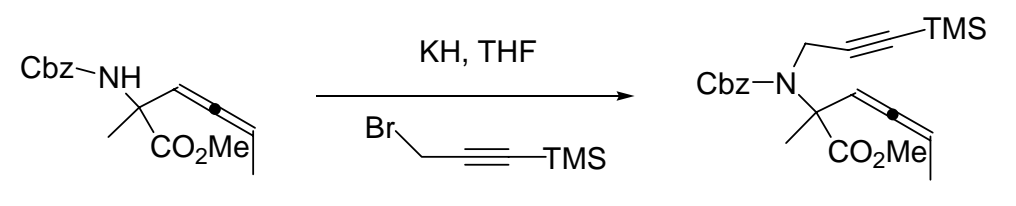

6, Table 1, Entry c

\section{2-[Benzyloxycarbonyl-(3-trimethylsilylprop-2-ynyl)-amino]-2-methylhexa-3,4-}

dienoic acid, methyl ester (6, Table 1, Entry c). To a solution of 2benzyloxycarbonylamino-2-methylhexa-3,4-dienoic acid, methyl ester (134 mg, 0.464 $\mathrm{mmol})$, in dry THF $(4 \mathrm{~mL})$ was added $\mathrm{KH}$ ( $68 \mathrm{mg}$ of $35 \%$ wt. dispersion in mineral oil, $0.51 \mathrm{mmol})$ at room temperature, followed by addition of (3-bromo-prop-1-ynyl)trimethylsilane $(131 \mu \mathrm{L}, 0.93 \mathrm{mmol})$. The reaction mixture was stirred at room temperature until TLC showed disappearance of the starting material $(10 \mathrm{~h})$. The reaction mixture was then poured into a beaker containing EtOAc $(50 \mathrm{~mL})$ and ice water $(50 \mathrm{~mL})$. The two layers were separated and the aqueous layer was extracted with EtOAc. The organic layers were then combined, washed with brine, dried over $\mathrm{MgSO}_{4}$, and concentrated under vacuum. Purified by flash chromatography (hexanes-EtOAc, $9: 1$, $\mathrm{v} / \mathrm{v}$ ) to afford the title compound ( $128 \mathrm{mg}, 86 \%$ ).

${ }^{1} \mathrm{H}$ NMR $\left(300 \mathrm{MHz}, \mathrm{CDCl}_{3}, 310 \mathrm{~K}\right) \delta$ 7.37-7.27 (m, 5H), $5.48-5.34(\mathrm{~m}, 2 \mathrm{H}), 5.20(1 / 2$ $\mathrm{AB}, J=12.5 \mathrm{~Hz}, 1 \mathrm{H}), 5.15(1 / 2 \mathrm{AB}, J=12.7 \mathrm{~Hz}, 1 \mathrm{H}), 4.38(1 / 2 \mathrm{AB}, J=18.3 \mathrm{~Hz}, 1 \mathrm{H})$, $4.18(1 / 2 \mathrm{AB}, J=18.4 \mathrm{~Hz}, 1 \mathrm{H}), 3.59(\mathrm{br} \mathrm{s}, 3 \mathrm{H}), 1.73(\mathrm{dd}, J=6.7,2.9 \mathrm{~Hz}, 3 \mathrm{H}), 1.66$ (s, $3 \mathrm{H}), 0.16(\mathrm{~s}, 9 \mathrm{H}) ;{ }^{13} \mathrm{C}$ NMR $\left(75 \mathrm{MHz}, \mathrm{CDCl}_{3}, 310 \mathrm{~K}\right) \delta 205.3,172.5 .155 .1,136.3$, $128.3,127.9,102.7,92.9,90.5,88.0,67.5,64.5,52.3,34.8,13.8,-0.3$; IR (thin film) $v$ 2954, 2177, 1967, 1748, 1707, $1251 \mathrm{~cm}^{-1}$; MS m/z (\%) 399 (5), 384 (9), 340 (25), 91 (100); EI-HRMS calcd for $\mathrm{C}_{22} \mathrm{H}_{29} \mathrm{NO}_{4} \mathrm{Si} m / z$ [M $]$ 399.1866; found 339.1875.

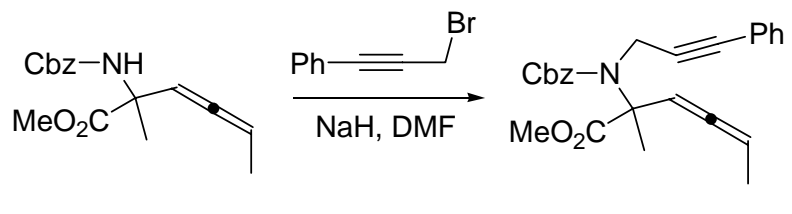

6, Table 1, Entry d

2-[Benzyloxycarbonyl-(3-phenylprop-2-ynyl)-amino]-2-methylhexa-3,4-dienoic acid, methyl ester (6, Table 1, Entry d). Followed general procedure for N-alkylation of Nprotected allenic amino acid methyl esters (page s12): 
2-Benzyloxycarbonylamino-2-methylhexa-3,4-dienoic acid, methyl ester (210 mg, 0.72 $\mathrm{mmol}$ ), $\mathrm{NaH}$ (58 $\mathrm{mg}$ of $60 \%$ dispersion in mineral oil, $1.44 \mathrm{mmol}$ ), (3-bromoprop-1ynyl)-benzene (281 mg, $1.44 \mathrm{mmol}$ )

Purified by flash chromatography (hexanes-EtOAc, $9: 1, \mathrm{v} / \mathrm{v}$ ) to afford the title compound (199 mg, 68\%).

${ }^{1} \mathrm{H}_{\mathrm{NMR}}\left(300 \mathrm{MHz}, \mathrm{DMSO}_{6}, 323 \mathrm{~K}\right) \delta$ 7.39-7.31 (m, $\left.10 \mathrm{H}\right), 5.55-5.45(\mathrm{~m}, 2 \mathrm{H}), 5.14$ (s, 2H), $4.52(1 / 2 \mathrm{AB}, J=18.6 \mathrm{~Hz}, 1 \mathrm{H}), 4.42(1 / 2 \mathrm{AB}, J=18.6 \mathrm{~Hz}, 1 \mathrm{H}), 3.50$ (bs, $3 \mathrm{H})$, $1.61(\mathrm{dd}, J=7.0,3.3 \mathrm{~Hz}, 3 \mathrm{H}), 1.59$ (s, $3 \mathrm{H}) ;{ }^{13} \mathrm{C}$ NMR $\left(75 \mathrm{MHz}, \mathrm{DMSO}-d_{6}, 323 \mathrm{~K}\right) \delta$ 204.2, 171.6, 154.4, 136.2, 130.9, 128.4, 128.3, 128.1, 127.6, 127.3, 122.0, 92.6, 90.2, 86.6, 82.5, 66.7, 63.5, 51.9, 34.1, 13.2; IR (thin film) v 2949, 1966, 1746, 1704, $1251 \mathrm{~cm}^{-}$ '; MS m/z (\%) 344 (39), 240 (40), 205 (49), 169 (61), 133 (84), 87 (100); EI-HRMS calcd for $\mathrm{C}_{25} \mathrm{H}_{25} \mathrm{NO}_{4} \mathrm{~m} / \mathrm{z}\left[\mathrm{M}^{+}\right] 403.1784$, found 403.1788 and calcd for $\mathrm{C}_{23} \mathrm{H}_{22} \mathrm{NO}_{2} \mathrm{~m} / \mathrm{z}$ [M-59 $]$ 344.1651 , found 344.1647 .

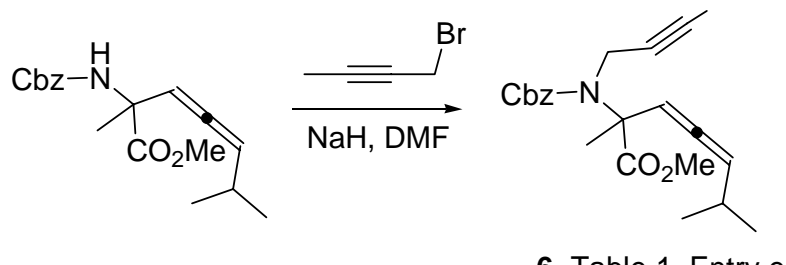

2-(Benzyloxycarbonylbut-2-ynylamino)-2,6-dimethylhepta-3,4-dienoic acid, methyl ester (6, Table 1, Entry e). Followed general procedure for N-alkylation of N-protected allenic amino acid methyl esters (page s12):

2-Benzyloxycarbonylamino-2,6-dimethylhepta-3,4-dienoic acid, methyl ester (125 mg, $0.394 \mathrm{mmol}$ ), $\mathrm{NaH}$ (31 $\mathrm{mg}$ of a $60 \%$ dispersion in mineral oil, $0.79 \mathrm{mmol}$ ), 1-bromo-2butyne $(69 \mu \mathrm{L}, 0.79 \mathrm{mmol})$.

Purification by flash chromatography (hexanes-EtOAc, $9: 1$, v/v) afforded the title compound (125 mg, 86\%).

${ }^{1} \mathrm{H}$ NMR (300 MHz, DMSO-d 6 , $\left.323 \mathrm{~K}\right) \delta$ 7.40-7.30 (m, 5H), 5.59-5.51 (m, 2H), $5.10(\mathrm{~s}$, 2H), $4.21(\mathrm{dq}, J=18.2,2.3 \mathrm{~Hz}, 1 \mathrm{H}), 4.11(\mathrm{dq}, J=18.3,2.4 \mathrm{~Hz}, 1 \mathrm{H}), 3.48(\mathrm{~s}, 3 \mathrm{H}), 2.40-$ $2.25(\mathrm{~m}, 1 \mathrm{H}), 1.75(\mathrm{t}, J=2.2 \mathrm{~Hz}, 3 \mathrm{H}), 1.52(\mathrm{~s}, 3 \mathrm{H}), 1.01(\mathrm{~d}, J=6.8 \mathrm{~Hz}, 6 \mathrm{H}) ;{ }^{13} \mathrm{C}$ NMR (75 MHz, DMSO-d $d_{6}, 323$ K) $\delta$ 201.6, 171.3, 154.3, 136.0, 127.9, 127.4, 127.1, 102.4, $94.4,78.5,75.9,66.5,63.5,51.6,33.7,27.0,21.7,21.5,2.5$; IR (thin film) v 2959, 1964, 
1746, 1704, $1253 \mathrm{~cm}^{-1}$; MS m/z (\%) 369 (24), 326 (18), 167 (20), 91 (50), 83 (100); EIHRMS calcd for $\mathrm{C}_{22} \mathrm{H}_{27} \mathrm{NO}_{4} \mathrm{~m} / \mathrm{z}\left[\mathrm{M}^{+}\right]$369.1940; found 369.1957.

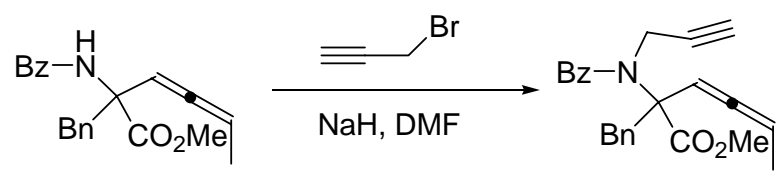

6, Table 1, Entry $f$

2-(Benzoylprop-2-ynylamino)-2-benzylhexa-3,4-dienoic acid, methyl ester (6, Table 1, Entry f). Followed general procedure for N-alkylation of N-protected allenic amino acid methyl esters (page s12):

2-Benzoylamino-2-benzyl-hexa-3,4-dienoic acid, methyl ester (175 mg, $0.521 \mathrm{mmol}$ ), $\mathrm{NaH}$ (60\% dispersion in mineral oil) (42 $\mathrm{mg}, 1.0 \mathrm{mmol}$ ), propargyl bromide ( $80 \% \mathrm{wt}$. in toluene) ( $92 \mu \mathrm{L}, 0.83 \mathrm{mmol})$.

Purification by flash chromatography (hexanes-EtOAc, $6: 1$, v/v) afforded the title compound (143 mg, 73\%).

${ }^{1} \mathrm{H}$ NMR (300 MHz, $\left.\mathrm{CDCl}_{3}\right)$ $\delta$ 7.67-7.62 (m, 2H), 7.49-7.39 (m, 3H), 7.28-7.23 (m, 5H), $5.85(\mathrm{sex}, J=3.1 \mathrm{~Hz}, 1 \mathrm{H}), 5.56-5.41(\mathrm{~m}, 1 \mathrm{H}), 3.97(1 / 2 \mathrm{AB}, J=13.5 \mathrm{~Hz}, 1 \mathrm{H}) *, 3.96(1 / 2$ $\mathrm{AB}, J=13.6 \mathrm{~Hz}, 1 \mathrm{H})^{* *}, 3.76(\mathrm{~s}, 3 \mathrm{H})^{* *}, 3.76(\mathrm{~s}, 3 \mathrm{H})^{*}, 3.76-3.75(\mathrm{~m}, 1 \mathrm{H})^{* *}, 3.70-3.68$ $(\mathrm{m}, 1 \mathrm{H})^{*}, 3.55-3.53(\mathrm{~m}, 1 \mathrm{H})^{*}, 3.48-3.47(\mathrm{~m}, 1 \mathrm{H})^{* *}, 3.42(1 / 2 \mathrm{AB}, J=13.6 \mathrm{~Hz}, 1 \mathrm{H})^{*}$, $3.41(1 / 2 \mathrm{AB}, \mathrm{J}=13.6 \mathrm{~Hz}, 1 \mathrm{H})^{* *}, 2.14(\mathrm{t}, J=2.4 \mathrm{~Hz}, 1 \mathrm{H})^{*}, 2.12(\mathrm{t}, J=2.4 \mathrm{~Hz}, 1 \mathrm{H})^{* *}$, $1.81(\mathrm{dd}, J=7.2,3.2 \mathrm{~Hz}, 3 \mathrm{H}) *, 1.75(\mathrm{dd}, J=7.1,3.2 \mathrm{~Hz}, 3 \mathrm{H}) * * ;{ }^{13} \mathrm{C} \mathrm{NMR}(75 \mathrm{MHz}$, $\left.\mathrm{CDCl}_{3}\right) \delta 205.0,172.7,171.7,136.7,136.6,136.2,131.4,130.7,128.7,128.4,127.5$, 127.1, 91.1, 91.0, 90.7, 81.2, 72.9, 72.9, 69.2, 52.7, 52.7, 39.3, 39.2, 14.1, 13.8; IR (thin film) $v 2948,2118,1968,1743,1643 \mathrm{~cm}^{-1}$; MS m/z (\%) 372 (50), 282 (40), 105 (100); EI-HRMS calcd for $\mathrm{C}_{24} \mathrm{H}_{22} \mathrm{NO}_{3} \mathrm{~m} / \mathrm{z}\left[\mathrm{M}^{+}\right] 372.1600$; found 372.1613 .

*diastereomer $1 ; * *$ diastereomer 2

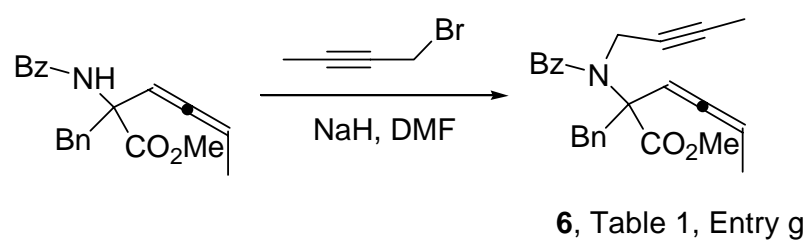

2-(Benzoyl-but-2-ynyl-amino)-2-benzylhexa-3,4-dienoic acid methyl ester (6, Table 1, Entry g). Followed general procedure for $\mathrm{N}$-alkylation of N-protected allenic amino acid methyl esters (page s12): 
2-Benzoylamino-2-benzyl-hexa-3,4-dienoic acid methyl ester (330 mg, $0.982 \mathrm{mmol}$ ), $\mathrm{NaH}$ (95\%) (60 mg, $2.5 \mathrm{mmol}), 1$-bromo-2-butyne ( $86 \mu \mathrm{L}, 0.98 \mathrm{mmol})$.

Purification by flash chromatography (hexanes-EtOAc, $9: 1$, v/v) afforded the title compound (315 mg, 83\%).

${ }^{1} \mathrm{H}$ NMR $\left(300 \mathrm{MHz}, \mathrm{CDCl}_{3}\right) \delta$ 7.67-7.64 (m, 2H), 7.48-7.38 (m, 3H), 7.31-7.21 (m, 5H), 5.89-85 (m, 1H), 5.52-5.38 (m, 1H), $3.98(1 / 2 \mathrm{AB}, J=13.6 \mathrm{~Hz}, 1 \mathrm{H})^{*}, 3.97(1 / 2 \mathrm{AB}, J=$ $13.5 \mathrm{~Hz}, 1 \mathrm{H})^{* *}, 3.75(\mathrm{~s}, 3 \mathrm{H}), 3.75-3.68(\mathrm{~m}, 1 \mathrm{H}), 3.58-3.51(\mathrm{~m}, 1 \mathrm{H}), 3.40(1 / 2 \mathrm{AB}, J=$ $13.6 \mathrm{~Hz}, 1 \mathrm{H})^{*}, 3.39(1 / 2 \mathrm{AB}, J=13.5 \mathrm{~Hz}, 1 \mathrm{H})^{* *}, 1.80(\mathrm{dd}, J=7.1,3.2 \mathrm{~Hz}, 3 \mathrm{H})^{*}, 1.74$ $(\mathrm{dd}, J=7.1,3.1 \mathrm{~Hz}, 3 \mathrm{H})^{* *}, 1.68(\mathrm{~s}, 3 \mathrm{H})$.

* from the major diastereomer

** from the minor diastereomer

${ }^{13} \mathrm{C}$ NMR $\left(75 \mathrm{MHz}, \mathrm{CDCl}_{3}\right) \delta 204.4,172.5,171.7,136.5,136.1,131.3,130.5,128.4$, $128.0,127.5,126.9,91.0,90.5,90.3,80.5,76.2,69.2,68.0,52.4,39.6,39.2,25.7,14.0$, 13.8, 3.7; IR (thin film) v 2948, 1967, 1744, 1642, $1393 \mathrm{~cm}^{-1}$; MS m/z (\%) 386 (10), 328 (41), 296 (15), 105 (100); EI-HRMS calcd for $\mathrm{C}_{25} \mathrm{H}_{24} \mathrm{NO}_{3} \mathrm{~m} / \mathrm{z}$ [M-1 $\left.{ }^{+}\right]$386.1756; found 386.1745 .
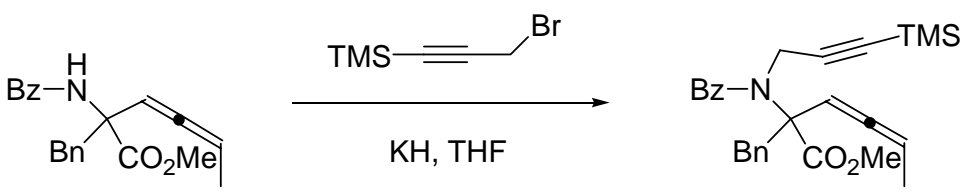

6, Table 1, Entry h

\section{2-[Benzoyl-(3-trimethylsilylprop-2-ynyl)-amino]-2-benzylhexa-3,4-dienoic acid}

methyl ester (6, Table 1, Entry h). To a solution of 2-benzoylamino-2-benzyl-hexa-3,4dienoic acid methyl ester (243 mg, $0.723 \mathrm{mmol})$, in dry THF $(7 \mathrm{~mL})$ was added $\mathrm{KH}$ (90 $\mathrm{mg}$ of $35 \%$ wt. dispersion in mineral oil, $0.8 \mathrm{mmol}$ ) at room temperature, followed by addition of (3-bromo-prop-1-ynyl)-trimethylsilane (203 $\mu \mathrm{L}, 1.44 \mathrm{mmol})$. The reaction mixture was stirred at room temperature until TLC showed disappearance of the starting material $(12 \mathrm{~h})$. The reaction mixture was then carefully poured into a beaker containing EtOAc and water. The two layers were separated and the aqueous layer was extracted with EtOAc. The organic layers were then combined, washed with brine $(20 \mathrm{~mL})$, dried with $\mathrm{MgSO}_{4}$, and concentrated under vacuum. Purification by flash chromatography (hexanes-EtOAc, $9: 1, \mathrm{v} / \mathrm{v}$ ) afforded the title compound (240 mg, 75\%). 
${ }^{1} \mathrm{H}$ NMR $\left(300 \mathrm{MHz}, \mathrm{CDCl}_{3}\right) \delta$ 7.68-7.64 (m, 2H), 7.46-7.38 (m, 3H), 7.33-7.21 (m, 5H), 5.83-5.80 (m, 1H), 5.57-5.41 (m, 1H), 3.98-3.91 (m, 1H), $3.76(\mathrm{~s}, 3 \mathrm{H})^{* *}, 3.75(\mathrm{~s}, 3 \mathrm{H})^{*}$, 3.67-3.65 (m, 1H), 3.52-3.41 (m, 2H), $1.81(\mathrm{dd}, J=7.2,3.1 \mathrm{~Hz}, 1 \mathrm{H})^{*}, 1.77(\mathrm{dd}, J=7.1$, $3.1 \mathrm{~Hz}, 1 \mathrm{H})^{* *}, 0.16(\mathrm{~s}, 9 \mathrm{H})^{*}, 0.15(\mathrm{~s}, 9 \mathrm{H})^{* *}$

* from the major diastereomer

** from the minor diastereomer

${ }^{13} \mathrm{C}$ NMR $\left(75 \mathrm{MHz}, \mathrm{CDCl}_{3}\right) \delta$ 204.6, 172.6, 171.5, 136.4, 136.3, 135.8, 130.9, 130.3, 128.2, 128.0, 127.3, 126.8, 103.2, 90.9, 90.4, 89.4, 68.8, 52.4, 39.8, 38.8, 38.5, 13.9, 13.7, -0.3; IR (thin film) v 2952, 2177, 1967, 1745, $1649 \mathrm{~cm}^{-1}$; MS m/z (\%) 445 (21), 444 (36), 354 (50), 244 (74), 105 (100); EI-HRMS calcd for $\mathrm{C}_{27} \mathrm{H}_{30} \mathrm{NO}_{3} \mathrm{Si} \mathrm{m} / \mathrm{z}\left[\mathrm{M}-1^{+}\right] 444.1995$; found 444.1984 .

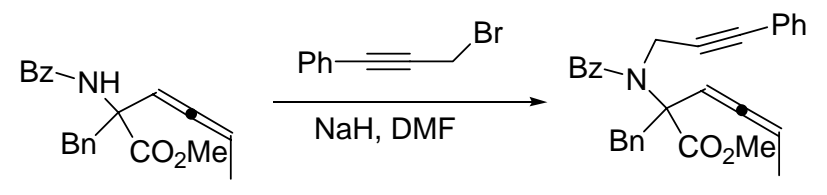

6, Table 1, Entry i

2-[Benzoyl-(3-phenylprop-2-ynyl)-amino]-2-benzylhexa-3,4-dienoic acid methyl ester (6, Table 1, Entry i). Followed general procedure for $\mathrm{N}$-alkylation of N-protected allenic amino acid methyl esters (page s12):

2-Benzoylamino-2-benzyl-hexa-3,4-dienoic acid methyl ester (72 mg, $0.21 \mathrm{mmol}), \mathrm{NaH}$ (60\% dispersion in mineral oil) (17 mg, $0.43 \mathrm{mmol})$, (3-bromo-prop-1-ynyl)-benzene (66 $\mathrm{mg}, 0.34 \mathrm{mmol})$.

Purification by flash chromatography (hexanes-EtOAc, $9: 1$, v/v) afforded the title compound ( $86 \mathrm{mg}, 89 \%$ ).

${ }^{1} \mathrm{H}$ NMR $\left(300 \mathrm{MHz}, \mathrm{CDCl}_{3}\right) \delta$ 7.79-7.77 (m, 2H), 7.57-7.51 (m, 4H), 7.41-7.23 (m, 9H), 5.98 (sept, $J=3.1 \mathrm{~Hz}, 1 \mathrm{H}), 5.65-5.50(\mathrm{~m}, 1 \mathrm{H}), 4.11(1 / 2 \mathrm{AB}, J=13.6 \mathrm{~Hz}, 1 \mathrm{H})^{*}, 4.11-$ $4.01(\mathrm{~m}, 2 \mathrm{H}), 3.87(\mathrm{~s}, 3 \mathrm{H})^{* *}, 3.85(\mathrm{~s}, 3 \mathrm{H})^{*}, 3.83-3.77(\mathrm{~m}, 1 \mathrm{H}), 3.54(\mathrm{~d}, J=13.5,1 \mathrm{H})$, $1.89(\mathrm{dd}, J=7.2,3.2 \mathrm{~Hz}, 3 \mathrm{H})^{*}, 1.83(\mathrm{dd}, J=7.1,3.2 \mathrm{~Hz}, 3 \mathrm{H})^{* *}$.

* from the major diastereomer

** from the minor diastereomer

${ }^{13} \mathrm{C}$ NMR $\left(75 \mathrm{MHz}, \mathrm{CDCl}_{3}\right) \delta 204.6,172.7,172.6,171.7,171.6,136.4,136.3,131.6$, $131.5,131.0,130.4,128.4,128.3,128.2,128.0,127.3,126.9,122.6,90.9,90.8,90.4$, 
$86.4,86.3,84.2,69.0,68.9,52.4,39.8,39.0,38.7,13.9,13.7$; IR (thin film) v 2948, 1967, 1743, 1645, $1392 \mathrm{~cm}^{-1}$; MS m/z (\%) 448 (7), 390 (78), 358 (25), 105 (100); EI-HRMS calcd for $\mathrm{C}_{30} \mathrm{H}_{26} \mathrm{NO}_{3} \mathrm{~m} / \mathrm{z}\left[\mathrm{M}-1^{+}\right]$448.1913; found 448.1929.

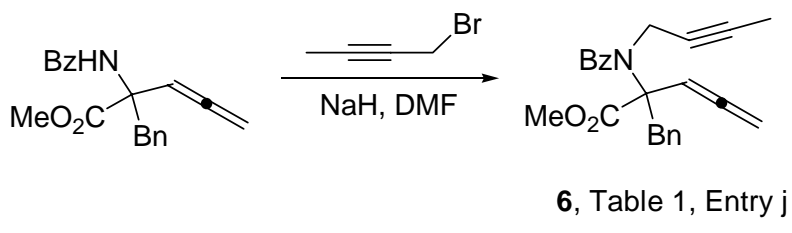

2-(Benzoylbut-2-ynylamino)-2-benzylpenta-3,4-dienoic acid methyl ester (6, Table 1, Entry j). Followed general procedure for N-alkylation of N-protected allenic amino acid methyl esters (page s12):

2-Benzoylamino-2-benzyl-penta-3,4-dienoic acid methyl ester (450 mg, $1.40 \mathrm{mmol}$ ), $\mathrm{NaH}(60 \%$ dispersion in mineral oil) $(123 \mathrm{mg}, 3.08 \mathrm{mmol})$, 1-bromo-2-butyne $(183 \mu \mathrm{L}$, $2.10 \mathrm{mmol})$.

Purification by flash chromatography (hexanes-EtOAc, $9: 1, \mathrm{v} / \mathrm{v}$ ) afforded the title compound (438 mg, 84\%).

${ }^{1} \mathrm{H}$ NMR $\left(300 \mathrm{MHz}, \mathrm{CDCl}_{3}\right) \delta$ 7.68-7.65 (m, 2H), 7.46-7.41 (m, 3H), 7.32-7.22 (m, 5H), $5.97(\mathrm{t}, J=6.6 \mathrm{~Hz}, 1 \mathrm{H}), 5.10(\mathrm{dd}, J=9.3,6.6 \mathrm{~Hz}, 1 \mathrm{H}), 5.05(\mathrm{dd}, J=9.3,6.6 \mathrm{~Hz}, 1 \mathrm{H})$, $3.98(1 / 2 \mathrm{AB}, J=13.6 \mathrm{~Hz}, 1 \mathrm{H}), 3.76$ (s, 3H), 3.73 (dq, $J=18.6,2.3 \mathrm{~Hz}, 1 \mathrm{H}), 3.55$ (dq, $J$ $=18.6,2.3 \mathrm{~Hz}, 1 \mathrm{H}), 3.43(1 / 2 \mathrm{AB}, J=13.6 \mathrm{~Hz}, 1 \mathrm{H}), 1.69(\mathrm{t}, J=2.3 \mathrm{~Hz}, 3 \mathrm{H}) ;{ }^{13} \mathrm{C} \mathrm{NMR}$ $\left(75 \mathrm{MHz}, \mathrm{CDCl}_{3}\right) \delta 207.6,172.4,171.3,136.2,135.9,131.1,130.3,128.2,128.2,127.9$, 127.3, 126.8, 90.8, 80.4, 79.2, 75.9, 68.7, 52.2, 39.4, 39.0, 3.5; IR (thin film) 2949, 2228, 1958, 1744, $1641 \mathrm{~cm}^{-1}$; MS m/z (\%) 372 (45), 342 (10), 282 (31), 105 (100); EI-HRMS calcd for $\mathrm{C}_{24} \mathrm{H}_{22} \mathrm{NO}_{3} \mathrm{~m} / \mathrm{z}\left[\mathrm{M}-1^{+}\right] 372.1600$, found 372.1618 .
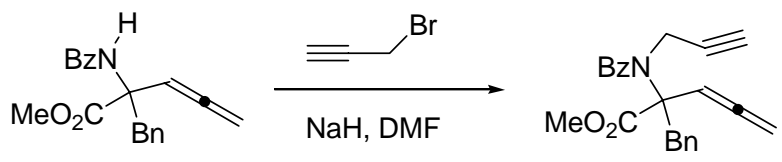

6, Table 1, Entry k

\section{2-(Benzoylprop-2-ynyl-amino)-2-benzylhexa-3,4-dienoic acid methyl ester (6, Table}

1, Entry k). Followed general procedure for N-alkylation of $\mathrm{N}$-protected allenic amino acid methyl esters (page s12): 
2-Benzoylamino-2-benzyl-penta-3,4-dienoic acid methyl ester (495 mg, $1.54 \mathrm{mmol}$ ), $\mathrm{NaH}$ (60\% dispersion in mineral oil) (154 mg, $3.85 \mathrm{mmol}$ ), propargyl bromide ( $80 \% \mathrm{wt}$. in toluene) $(257 \mu \mathrm{L}, 2.31 \mathrm{mmol})$.

Purification by flash chromatography (gradient elution; hexanes-EtOAc, $9: 1$ to $4: 1$, $\mathrm{v} / \mathrm{v}$ ) afforded the title compound (364 mg, 66\%).

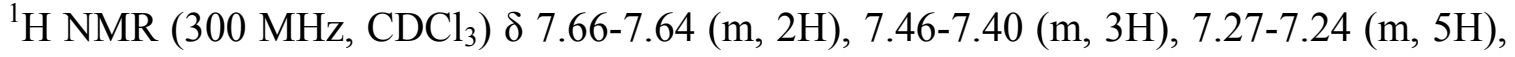
$5.94(\mathrm{t}, J=6.6 \mathrm{~Hz}, 1 \mathrm{H}), 5.10$ (app dd, $J=6.6,2.9 \mathrm{~Hz}, 2 \mathrm{H}), 3.96(1 / 2 \mathrm{AB}, J=13.6 \mathrm{~Hz}$, 1H), $3.76(\mathrm{~s}, 3 \mathrm{H}), 3.73$ (dd, $J=18.8,1.8 \mathrm{~Hz}, 1 \mathrm{H}), 3.51(\mathrm{dd}, J=18.8,1.9 \mathrm{~Hz}, 1 \mathrm{H}), 3.45$ $(1 / 2 \mathrm{AB}, J=13.7 \mathrm{~Hz}, 1 \mathrm{H}), 2.18(\mathrm{~s}, 1 \mathrm{H}) ;{ }^{13} \mathrm{C} \mathrm{NMR}\left(75 \mathrm{MHz}, \mathrm{CDCl}_{3}\right) \delta 207.7,172.4$, $171.1,135.9,135.5,130.9,130.4,128.3,128.1,127.1,126.9,90.5,80.5,79.6,72.8,68.5$, $52.3,38.8,38.6$. IR (thin film) v 3302, 2950, 2115, 1957, 1742, 1640, 1433, 1398, 1266, $1246 \mathrm{~cm}^{-1}$; MS m/z (\%) 358 (35), 268 (42), 105 (100); EI HRMS calcd for $\mathrm{C}_{23} \mathrm{H}_{20} \mathrm{NO}_{3}$ $\mathrm{m} / \mathrm{z}\left[\mathrm{M}^{+}\right]$358.1443, found 358.1451 .

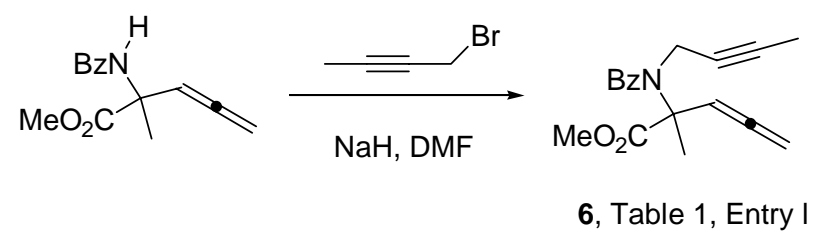

2-(Benzoylbut-2-ynyl-amino)-2-methylpenta-3,4-dienoic acid methyl ester (6, Table

1, Entry l). Followed general procedure for N-alkylation of N-protected allenic amino acid methyl esters (page s12):

2-Benzoylamino-2-methyl-penta-3,4-dienoic acid methyl ester (200 mg, $0.816 \mathrm{mmol}$ ), $\mathrm{NaH}(65 \mathrm{mg}$ of a $60 \%$ dispersion in mineral oil, $1.6 \mathrm{mmol})$, 1-bromo-2-butyne (142 $\mu \mathrm{L}, 1.6 \mathrm{mmol})$.

Purification by flash chromatography (gradient elution; hexanes-EtOAc, $9: 1$ to $4: 1$, $\mathrm{v} / \mathrm{v}$ ) afforded the title compound ( $226 \mathrm{mg}, 93 \%$ ).

${ }^{1} \mathrm{H}$ NMR (300 MHz, $\left.\mathrm{CDCl}_{3}\right) \delta$ 7.60-7.57 (m, 2H), 7.40-7.34 (m, 3H), $5.79(\mathrm{t}, J=6.6 \mathrm{~Hz}$, $1 \mathrm{H}), 4.95$ (d, $J=6.6 \mathrm{~Hz}, 1 \mathrm{H}), 3.96$ (q, $J=2.2 \mathrm{~Hz}, 2 \mathrm{H}), 3.71(\mathrm{~s}, 3 \mathrm{H}), 1.82$ (t, $J=2.2 \mathrm{~Hz}$, $3 \mathrm{H}), 1.72$ (s, 3H); ${ }^{13} \mathrm{C}$ NMR (75MHz, $\left.\mathrm{CDCl}_{3}\right) \delta 208.2,172.2,171.8,135.5,130.2,128.1$, $128.1,127.0,92.5,80.3,78.9,75.8,63.4,52.4,43.0,37.3,20.5,3.4$; IR (thin film) $v$ 2949, 1957, 1742, 1637, 1398, $1264 \mathrm{~cm}^{-1}$; MS m/z (\%) 297 (19), 258 (20), 238 (52), 105 (100); EI HRMS calcd for $\mathrm{C}_{18} \mathrm{H}_{19} \mathrm{NO}_{3} \mathrm{~m} / z$ [ $\left[\mathrm{M}^{+}\right]$297.1365, found 297.1359 . 


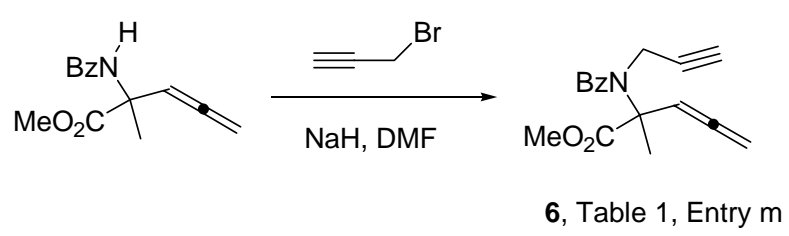

\section{2-(Benzoylprop-2-ynylamino)-2-methylpenta-3,4-dienoic acid methyl ester (6, Table}

1, Entry m). Followed general procedure for N-alkylation of N-protected allenic amino acid methyl esters (page s12):

2-Benzoylamino-2-methyl-penta-3,4-dienoic acid methyl ester (200 mg, $0.816 \mathrm{mmol})$, $\mathrm{NaH}$ (65 mg of a $60 \%$ dispersion in mineral oil, $1.6 \mathrm{mmol})$, propargyl bromide $(80 \% \mathrm{wt}$. in toluene) $(180 \mu \mathrm{L}, 1.6 \mathrm{mmol})$.

Purification by flash chromatography (gradient elution; hexanes-EtOAc, $9: 1$ to $4: 1$, $\mathrm{v} / \mathrm{v}$ ) afforded the title compound (217 $\mathrm{mg}, 94 \%)$.

${ }^{1} \mathrm{H}$ NMR $\left(300 \mathrm{MHz}, \mathrm{CDCl}_{3}\right) \delta$ 7.64-7.61 (m, 2H), 7.46-7.40 (m, 3H), $5.82(\mathrm{t}, J=6.6 \mathrm{~Hz}$, $1 \mathrm{H}), 5.01(\mathrm{~d}, J=6.5 \mathrm{~Hz}, 2 \mathrm{H}), 4.05$ (d, $J=2.4 \mathrm{~Hz}, 2 \mathrm{H}), 3.76(\mathrm{~s}, 3 \mathrm{H}), 2.39$ (t, $J=2.4 \mathrm{~Hz}$, $1 \mathrm{H}), 1.77(\mathrm{~s}, 3 \mathrm{H}) ;{ }^{13} \mathrm{C} \mathrm{NMR}\left(75 \mathrm{MHz}, \mathrm{CDCl}_{3}\right) \delta 208.5,172.2,172.1,135.5,130.4,128.4$, 127.1, 92.6, 80.8, 79.2, 72.8, 63.8, 52.6, 37.1, 20.7; IR (thin film) v 3256, 2950, 2115, 1956, 1740, 1640, 1397, $1264 \mathrm{~cm}^{-1}$; MS m/z (\%) 283 (20), 252 (24), 244 (26), 224 (51), 105 (100); EI HRMS calcd for $\mathrm{C}_{17} \mathrm{H}_{17} \mathrm{NO}_{3} \mathrm{~m} / \mathrm{z}\left[\mathrm{M}^{+}\right]$283.1208, found 283.1198.
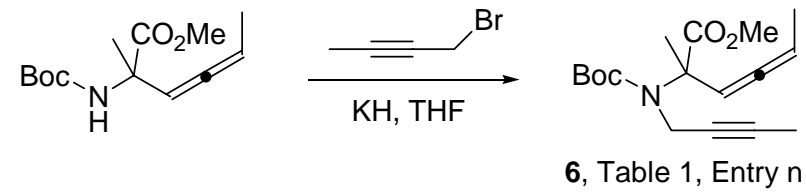

2-(tert-Butoxycarbonyl-but-2-ynylamino)-2-methylhexa-3,4-dienoic acid methyl ester (6, Table 1, Entry n). Followed general procedure for N-alkylation of N-protected allenic amino acid methyl esters (page s12) with the following modifications (KH was used as a base instead of $\mathrm{NaH}$, and THF was used as solvent instead of DMF):

2-tert-butoxycarbonylamino-2-methyl-hexa-3,4-dienoic acid methyl ester (225 mg, 0.882 $\mathrm{mmol}), \mathrm{KH}$ (233 $\mathrm{mg}$ of 30\% wt. dispersion in mineral oil, $1.77 \mathrm{mmol}$ ), 1-bromo-2-butyne $(155 \mu \mathrm{L}, 1.77 \mathrm{mmol})$, THF $(10 \mathrm{~mL})$. After addition of the reagents at $0{ }^{\circ} \mathrm{C}$, the reaction was stirred for $1 \mathrm{~h}$ followed by $2 \mathrm{~h}$ at room temperature.

Purification by flash chromatography (gradient elution; hexanes-EtOAc, $19: 1$ to $9: 1$, v/v) afforded the title compound (173 mg, 64\%). 
${ }^{1} \mathrm{H}$ NMR (300 MHz, $\left.\mathrm{CDCl}_{3}\right) \delta$ 5.46-5.39 (m, 1H), 5.36-5.27 (m, 1H), $4.18(1 / 2 \mathrm{AB}, J=$ $17.6 \mathrm{~Hz}, 1 \mathrm{H}), 4.00(1 / 2 \mathrm{AB}, J=18.1,1.9 \mathrm{~Hz}, 1 \mathrm{H}), 3.66(\mathrm{~s}, 3 \mathrm{H}), 1.74(\mathrm{t}, J=2.3 \mathrm{~Hz}, 3 \mathrm{H})$, $1.68(\mathrm{dd}, J=7.1,3.2 \mathrm{~Hz}, 3 \mathrm{H}), 1.55(\mathrm{~s}, 3 \mathrm{H}), 1.40(\mathrm{~s}, 9 \mathrm{H}) ;{ }^{13} \mathrm{C} \mathrm{NMR}\left(75 \mathrm{MHz}, \mathrm{CDCl}_{3}\right) \delta$ 204.9, 173.0, 154.3, 93.0, 90.1, 81.0, 78.1, 76.1, 63.6, 52.2, 33.9, 28.1, 13.7, 3.4; MS m/z (\%) 251 (65), 192 (92), 154 (100), 148 (73); EI HRMS calcd for $\mathrm{C}_{13} \mathrm{H}_{17} \mathrm{NO}_{4} \mathrm{~m} / \mathrm{z}[\mathrm{M}+1$ $\left.\mathrm{tBu}^{+}\right]$251.1158, found 251.1169.

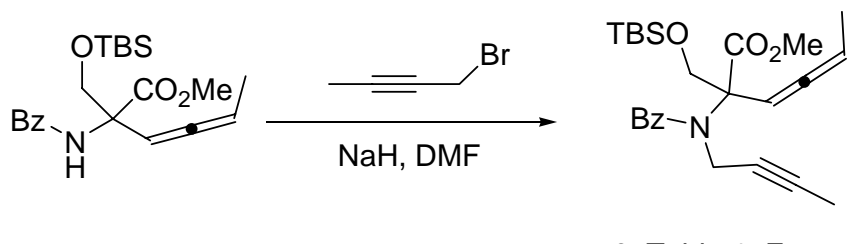

2-(Benzoyl-but-2-ynylamino)-2-(tert-butyldimethylsilyloxymethyl)-hexa-3,4-dienoic

acid methyl ester (6, Table 1, Entry o). Followed general procedure for N-alkylation of N-protected allenic amino acid methyl esters (page s12):

2-Benzoylamino-2-(tert-butyl-dimethyl-silyloxymethyl)-hexa-3,4-dienoic acid methyl ester (245 mg, $0.629 \mathrm{mmol}), \mathrm{NaH}(60 \%$ dispersion in mineral oil) (50 $\mathrm{mg}$ of a $60 \%$ dispersion in mineral oil, $1.3 \mathrm{mmol}), 1$-bromo-2-butyne ( $88 \mu \mathrm{L}, 1.1 \mathrm{mmol})$.

Purification by flash chromatography (gradient elution; hexanes-EtOAc, $19: 1$ to $9: 1$, $\mathrm{v} / \mathrm{v}$ ) afforded the title compound ( $237 \mathrm{mg}, 85 \%$ ).

${ }^{1} \mathrm{H}$ NMR $\left(300 \mathrm{MHz}, \mathrm{CDCl}_{3}\right) \delta$ 7.71-7.65 (m, 2H), 7.46-7.37 (m, 3H), 5.90-5.86 (m, 1H), 5.41-5.31 (m, 1H), $4.45(\mathrm{~d}, J=10.1 \mathrm{~Hz}, 1 \mathrm{H})^{* *}, 4.44(\mathrm{~d}, J=10.1 \mathrm{~Hz}, 1 \mathrm{H})^{*}, 4.31-4.16(\mathrm{~m}$, 2H), 3.99 (dq, $J=18.5,2.3 \mathrm{~Hz}, 1 \mathrm{H}), 3.74(\mathrm{~s}, 3 \mathrm{H})^{*}, 3.73(\mathrm{~s}, 3 \mathrm{H})^{* *}, 1.90-1.87(\mathrm{~m}, 3 \mathrm{H})$, $1.71(\mathrm{dd}, J=7.1,3.2 \mathrm{~Hz}, 3 \mathrm{H})^{*}, 1.71(\mathrm{dd}, J=7.1,3.2 \mathrm{~Hz}, 3 \mathrm{H})^{* *}, 0.87$ (s, 9H), 0.06 (s, $3 \mathrm{H}), 0.05(\mathrm{~s}, 3 \mathrm{H})$.

* minor diastereomer, $* *$ major diastereomer

${ }^{13} \mathrm{C}$ NMR $\left(75 \mathrm{MHz}, \mathrm{CDCl}_{3}\right) \delta 205.1,205.0,172.3,172.2,170.6,136.1,130.1,128.1$, $127.3,127.3,89.9,89.8,89.7,79.8,79.7,77.2,68.3,68.2,64.0,63.9,52.2,52.1,39.4$, $39.3,25.8,25.7,18.1,14.0,13.7,3.6,-5.5,-5.6,-5.6,-5.7$; IR (thin film) v 2952, 1968, 1744, $1644 \mathrm{~cm}^{-1}$; MS m/z (\%) 384 (35), 296 (31), 84 (100); EI-HRMS calcd for $\mathrm{C}_{21} \mathrm{H}_{26} \mathrm{NO}_{4} \mathrm{Si} \mathrm{m} / z\left[\mathrm{M}-57^{+}\right]$384.1631; found 384.1630. 
General procedure for the rhodium catalyzed formal Alder-ene reaction.
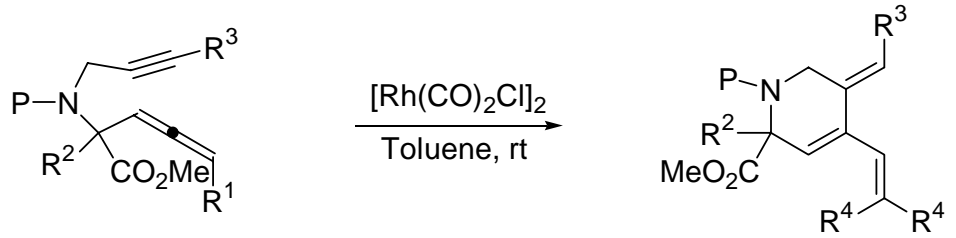

To a $10 \mathrm{~mL}$ test tube equipped with a magnetic stirring bar was added the alkynyl allene $(0.1 \mathrm{mmol})$ as a solution in benzene. The solvent was removed under vacuum and the atmosphere replaced with $\mathrm{N}_{2}$. Toluene $(1 \mathrm{~mL})$ was added followed by addition of $\left[\mathrm{Rh}(\mathrm{CO})_{2} \mathrm{Cl}\right]_{2}(0.005 \mathrm{mmol})$ at room temperature. Upon completion of the reaction based upon TLC $(10 \mathrm{~min})$ the solution was applied to a plug of silica gel. Elution with (hexanes-EtOAc, $9: 1$, v/v) afforded the title compound.
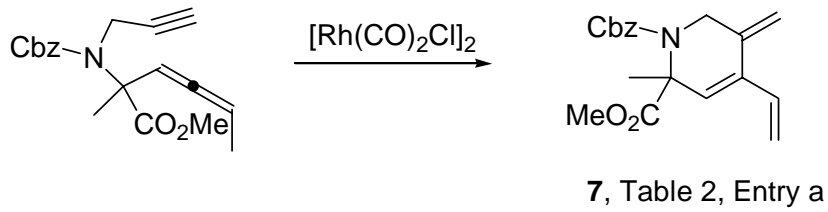

2-Methyl-5-methylene-4-vinyl-5,6-dihydro-2H-pyridine-1,2-dicarboxylic acid 1benzyl ester 2-methyl ester (7, Table 2, Entry a). Followed general procedure for the rhodium catalyzed formal Alder-ene reaction (page s23):

2-(Benzyloxycarbonylprop-2-ynylamino)-2-methylhexa-3,4-dienoic acid methyl ester (37 $\mathrm{mg}, 0.11 \mathrm{mmol}),\left[\mathrm{Rh}(\mathrm{CO})_{2} \mathrm{Cl}\right]_{2}(2.0 \mathrm{mg}, 0.005 \mathrm{mmol})$.

Yield (31 mg, 84\%).

${ }^{1} \mathrm{H}$ NMR $\left(300 \mathrm{MHz}\right.$, DMSO-d $\left.d_{6}, 323 \mathrm{~K}\right) \delta 7.35(\mathrm{~m}, 5 \mathrm{H}), 6.43(\mathrm{dd}, J=17.3,11.0 \mathrm{~Hz}, 1 \mathrm{H})$, $5.68(\mathrm{~s}, 1 \mathrm{H}), 5.48(\mathrm{~d}, J=17.3 \mathrm{~Hz}, 1 \mathrm{H}), 5.26-5.24(\mathrm{~m}, 2 \mathrm{H}), 5.11(\mathrm{~s}, 2 \mathrm{H}), 4.39(1 / 2 \mathrm{AB}, J=$ $14.2 \mathrm{~Hz}, 1 \mathrm{H}), 3.88$ (1/2 AB, J = 14.2 Hz, 1H), 3.49 (br s, 3H), 1.55 (s, 3H); NMR (75 MHz, DMSO- $d_{6}, 323$ K) $\delta 171.3,154.0,136.1,135.8,134.2,132.9,128.1,127.6,127.4$, $126.5,117.3,112.6,66.5,62.0,52.0,45.6,21.5$; IR (thin film) $\vee 2950,1743,1704,1247$ $\mathrm{cm}^{-1}$; MS m/z (\%) 327 (12), 268 (85), 224 (59), 91 (100); EI-HRMS calcd for $\mathrm{C}_{19} \mathrm{H}_{21} \mathrm{NO}_{4}$ $\mathrm{m} / \mathrm{z}\left[\mathrm{M}^{+}\right]$327.1471; found 327.1473.
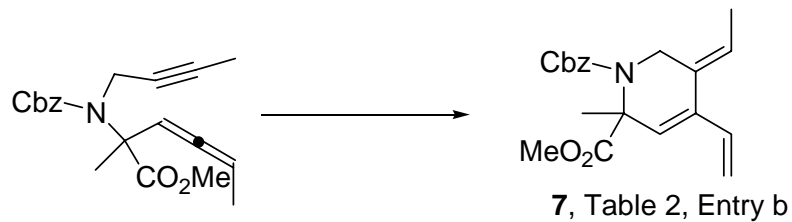
5-Ethylidene-2-methyl-4-vinyl-5,6-dihydro-2H-pyridine-1,2-dicarboxylic acid 1benzyl ester 2-methyl ester (7, Table 2, Entry b). Followed general procedure for the rhodium catalyzed formal Alder-ene reaction (page s23):

2-(Benzyloxycarbonyl-but-2-ynyl-amino)-2-methylhexa-3,4-dienoic acid methyl ester (515 mg, $1.51 \mathrm{mmol}),\left[\mathrm{Rh}(\mathrm{CO})_{2} \mathrm{Cl}\right]_{2}(30 \mathrm{mg}, 0.077 \mathrm{mmol})$.

Yield (420 mg, 81\%).

${ }^{1} \mathrm{H}$ NMR $\left(300 \mathrm{MHz}\right.$, DMSO-d $\left.d_{6}, 323 \mathrm{~K}\right) \delta$ 7.37-7.33 (m, $\left.5 \mathrm{H}\right), 6.40(\mathrm{dd}, J=17.2,10.8 \mathrm{~Hz}$ 1H), $5.76(\mathrm{q}, J=7.0 \mathrm{~Hz}, 1 \mathrm{H}), 5.50(\mathrm{br} \mathrm{s}, 1 \mathrm{H}), 5.40$ (dd, $J=17.2,1.3 \mathrm{~Hz} 1 \mathrm{H}), 5.19$ (dd, $J$ $=10.8,1.3 \mathrm{~Hz}, 1 \mathrm{H}), 5.15(1 / 2 \mathrm{AB}, J=12.4 \mathrm{~Hz}, 1 \mathrm{H}), 5.10(1 / 2 \mathrm{AB}, J=12.4 \mathrm{~Hz}, 1 \mathrm{H}), 4.58$ $(1 / 2 \mathrm{AB}, J=14.6 \mathrm{~Hz}, 1 \mathrm{H}), 3.79(1 / 2 \mathrm{AB}, J=14.6 \mathrm{~Hz}, 1 \mathrm{H}), 3.49$ (s, 3H), 1.76 (d, $J=7.0$ $\mathrm{Hz}, 3 \mathrm{H}), 1.55$ (s, $3 \mathrm{H}) ;{ }^{13} \mathrm{C}$ NMR $\left(75 \mathrm{MHz}\right.$, DMSO-d $\left.d_{6}, 323 \mathrm{~K}\right) \delta 171.5,154.1,136.1$, 135.3, 133.6, 128.9, 128.0, 127.5, 127.2, 123.9, 122.2, 117.0, 66.4, 61.6, 51.8, 12.7; IR (neat) $v$ 2949, 1743, 1704, 1404, 1243, $1110 \mathrm{~cm}^{-1}$; MS m/z (\%) 341 (26), 282 (26), 238 (15), 91 (100); EI-HRMS calcd for $\mathrm{C}_{20} \mathrm{H}_{23} \mathrm{NO}_{4}\left[\mathrm{M}^{+}\right] \mathrm{m} / \mathrm{z}$ 341.1621, found 341.1627.

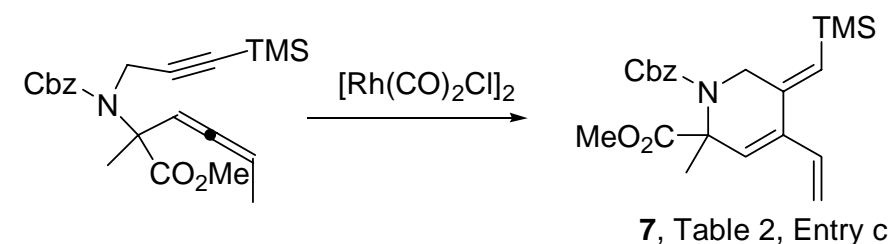

2-Methyl-5-trimethylsilylmethylene-4-vinyl-5,6-dihydro-2H-pyridine-1,2-

dicarboxylic acid 1-benzyl ester 2-methyl ester (7, Table 2, Entry c). Followed general procedure for the rhodium catalyzed formal Alder-ene reaction (page s23):

2-[Benzyloxycarbonyl-(3-trimethylsilylprop-2-ynyl)-amino]-2-methylhexa-3,4-dienoic acid methyl ester (30 mg, $0.075 \mathrm{mmol}),\left[\mathrm{Rh}(\mathrm{CO})_{2} \mathrm{Cl}\right]_{2}(1.5 \mathrm{mg}, 0.0037 \mathrm{mmol})$.

Yield (26 mg, 87\%).

${ }^{1} \mathrm{H}_{\mathrm{NMR}}(300 \mathrm{MHz}$, DMSO-d $6,323 \mathrm{~K}) \delta$ 7.39-7.30 (m, 5H), 6.43 (dd, $J=17.2,10.8 \mathrm{~Hz}$, $1 \mathrm{H}), 5.76(\mathrm{~s}, 1 \mathrm{H}), 5.66(\mathrm{~s}, 1 \mathrm{H}), 5.41(\mathrm{~d}, J=17.2 \mathrm{~Hz}, 1 \mathrm{H}), 5.21(\mathrm{dd}, J=10.8,0.7 \mathrm{~Hz}, 1 \mathrm{H})$, $5.13(1 / 2 \mathrm{AB}, J=13.4 \mathrm{~Hz}, 1 \mathrm{H}), 5.07(1 / 2 \mathrm{AB}, J=13.4 \mathrm{~Hz}, 1 \mathrm{H}), 4.54(1 / 2 \mathrm{AB}, J=14.4$ $\mathrm{Hz}, 1 \mathrm{H}), 3.94(1 / 2 \mathrm{AB}, J=14.4 \mathrm{~Hz}, 1 \mathrm{H}), 3.52(\mathrm{~s}, 3 \mathrm{H}), 1.57$ (s, 3H), $0.13(\mathrm{~s}, 9 \mathrm{H}) ;{ }^{13} \mathrm{C}$ NMR $\left(75 \mathrm{MHz}, \mathrm{DMSO}-d_{6}, 323 \mathrm{~K}\right) \delta 171.2,153.9,143.4,136.1,135.9,123.3,128.0$, $127.6,127.5,126.9,126.8,126.1117 .3,66.6,61.7,51.9,44.1,21.2,-0.5$; IR (neat) $v$ 
2953, 1745, 1706, 1405, 1248, $1113 \mathrm{~cm}^{-1}$; MS m/z (\%) 399 (25), 340 (34), 296 (15), 91 (100); EI-HRMS calcd for $\mathrm{C}_{22} \mathrm{H}_{29} \mathrm{NO}_{4} \mathrm{Si}\left[\mathrm{M}^{+}\right] \mathrm{m} / \mathrm{z}$ 399.1866, found 399.1843 .

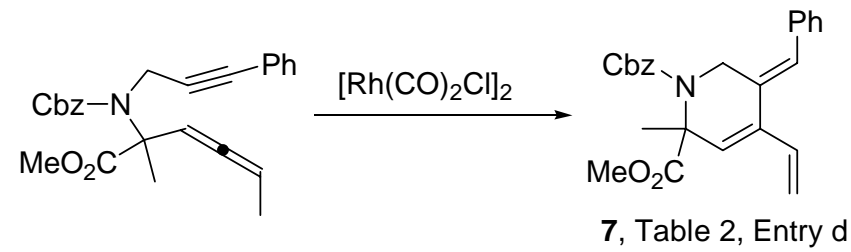

5-Benzylidene-2-methyl-4-vinyl-5,6-dihydro-2H-pyridine-1,2-dicarboxylic acid 1benzyl ester 2-methyl ester (7, Table 2, Entry d). Followed general procedure for the rhodium catalyzed formal Alder-ene reaction (page s23):

2-[Benzyloxycarbonyl-(3-phenylprop-2-ynyl)-amino]-2-methylhexa-3,4-dienoic acid methyl ester $(25 \mathrm{mg}, 0.062 \mathrm{mmol}),\left[\mathrm{Rh}(\mathrm{CO})_{2} \mathrm{Cl}\right]_{2}(1.0 \mathrm{mg}, 0.003 \mathrm{mmol})$.

Yield (25 mg, >95\%).

${ }^{1} \mathrm{H}$ NMR $\left(300 \mathrm{MHz}\right.$, DMSO- $\left.d_{6}, 323 \mathrm{~K}\right) \delta$ 7.46-7.21 (m, 10H), 6.76 (s, 1H), 6.57 (dd, $J=$ 17.1, $10.7 \mathrm{~Hz}, 1 \mathrm{H}), 5.72(\mathrm{~s}, 1 \mathrm{H}), 5.52(\mathrm{~d}, J=17.2 \mathrm{~Hz}, 1 \mathrm{H}), 5.29(\mathrm{~d}, J=10.8 \mathrm{~Hz}, 1 \mathrm{H})$, $5.07(1 / 2 \mathrm{AB}, J=12.6 \mathrm{~Hz}, 1 \mathrm{H}), 5.01(1 / 2 \mathrm{AB}, J=12.7 \mathrm{~Hz}, 1 \mathrm{H}), 4.87(1 / 2 \mathrm{AB}, J=14.7$ $\mathrm{Hz}, 1 \mathrm{H}), 4.09(1 / 2 \mathrm{AB}, J=14.7 \mathrm{~Hz}, 1 \mathrm{H}), 3.52$ (s, 3H), 1.60 (s, 3H); ${ }^{13} \mathrm{C} \mathrm{NMR}(75 \mathrm{MHz}$, DMSO- $\left._{6}, 323 \mathrm{~K}\right) \delta 171.4,153.9,135.9,135.9,135.7,133.7,130.0,128.8,128.1,127.6$, 127.2, 127.1, 126.9, 126.0, 117.8, 66.4, 61.8, 52.1, 21.3; IR (thin film) v 2949, 1742, 1704, 1408, $1248 \mathrm{~cm}^{-1}$; MS m/z (\%) 403 (37), 344 (45), 300 (17), 91 (35), 84 (100); EIHRMS calcd for $\mathrm{C}_{25} \mathrm{H}_{25} \mathrm{NO}_{4} \mathrm{~m} / \mathrm{z}\left[\mathrm{M}^{+}\right] 403.1784$, found 403.1789 .
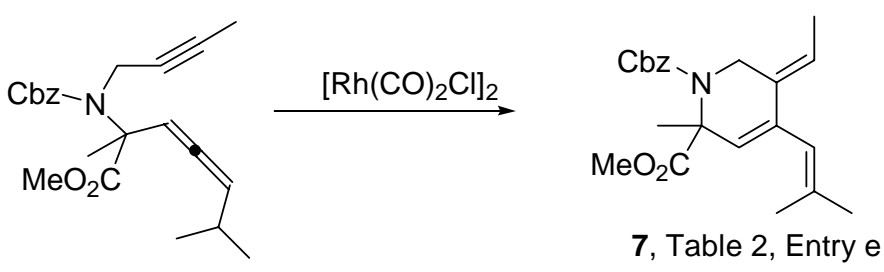

\section{5-Ethylidene-2-methyl-4-(2-methylpropenyl)-5,6-dihydro-2H-pyridine-1,2-}

dicarboxylic acid 1-benzylester 2-methyl ester (7, Table 2, Entry e). Followed general procedure for the rhodium catalyzed formal Alder-ene reaction (page s23):

2-(Benzyloxycarbonylbut-2-ynylamino)-2,6-dimethylhepta-3,4-dienoic acid methyl ester (40 mg, $0.11 \mathrm{mmol}),\left[\mathrm{Rh}(\mathrm{CO})_{2} \mathrm{Cl}\right]_{2}(2 \mathrm{mg}, 0.005 \mathrm{mmol})$.

Yield (40 mg, >95\%). 
${ }^{1} \mathrm{H}$ NMR $\left(300 \mathrm{MHz}, \mathrm{DMSO}-d_{6}, 323 \mathrm{~K}\right) \delta 7.35$ (s, 5H), $5.64(\mathrm{~s}, 1 \mathrm{H}), 5.58(\mathrm{q}, J=6.9 \mathrm{~Hz}$, $1 \mathrm{H}), 5.12(\mathrm{~s}, 3 \mathrm{H}), 4.65(1 / 2 \mathrm{AB}, J=14.9 \mathrm{~Hz}, 1 \mathrm{H}), 3.71(1 / 2 \mathrm{AB}, J=14.7 \mathrm{~Hz}, 1 \mathrm{H}), 3.47$ (br s, 3H), $1.77(\mathrm{~s}, 3 \mathrm{H}), 1.72(\mathrm{~d}, J=6.8 \mathrm{~Hz}, 3 \mathrm{H}), 1.58(\mathrm{~s}, 3 \mathrm{H}), 1.52(\mathrm{~s}, 3 \mathrm{H}) ;{ }^{13} \mathrm{C} \mathrm{NMR}(75$ MHz, DMSO-d $d_{6}, 323$ K) $\delta 171.9,154.2,137.2$, 136.2, 134.2, 130.2, 128.1, 128.1, 127.7, $127.4,125.5,122.0,121.1,66.5,61.5,51.8,25.3,18.5,12.8$; IR (thin film) v 2930, 1742, 1687, 1424, $1253 \mathrm{~cm}^{-1}$; MS m/z (\%) 369 (10), 310 (50), 266 (36), 91 (100); EI-HRMS calcd for $\mathrm{C}_{22} \mathrm{H}_{27} \mathrm{NO}_{4} \mathrm{~m} / \mathrm{z}\left[\mathrm{M}^{+}\right]$369.1940; found 369.1952.

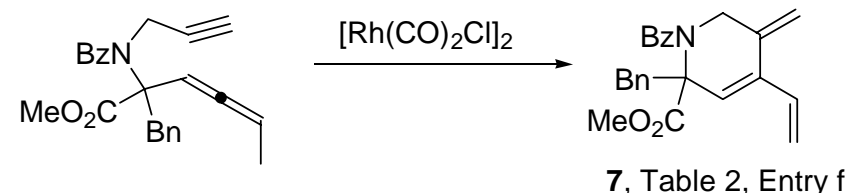

\section{1-Benzoyl-2-benzyl-5-methylene-4-vinyl-1,2,5,6-tetrahydropyridine-2-carboxylic}

acid methyl ester (7, Table 2, Entry f). Followed general procedure for the rhodium catalyzed formal Alder-ene reaction (page s23):

2-(Benzoylprop-2-ynylamino)-2-benzylhexa-3,4-dienoic acid methyl ester (29 mg, 0.078 $\mathrm{mmol}),\left[\mathrm{Rh}(\mathrm{CO})_{2} \mathrm{Cl}\right]_{2}(1.5 \mathrm{mg}, 0.0039 \mathrm{mmol})$.

Yield (21 mg, 74\%).

${ }^{1} \mathrm{H}$ NMR $\left(300 \mathrm{MHz}, \mathrm{CDCl}_{3}\right) \delta$ 7.44-7.38 (m, $\left.5 \mathrm{H}\right), 7.25-7.20(\mathrm{~m}, 5 \mathrm{H}), 6.37(\mathrm{dd}, J=17.3$, $10.8 \mathrm{~Hz}, 1 \mathrm{H}), 5.85$ (s, 1H), 5.52 (dd, $J=17.3,1.2 \mathrm{~Hz}, 1 \mathrm{H}), 5.28(\mathrm{dd}, J=10.8,1.1 \mathrm{~Hz}$, 1H), 5.09 (s, 1H), $4.76(\mathrm{~s}, 1 \mathrm{H}), 4.10(1 / 2 \mathrm{AB}, J=13.7,1 \mathrm{H}), 3.84(1 / 2 \mathrm{AB}, J=13.9,1 \mathrm{H})$, $3.77(\mathrm{~s}, 3 \mathrm{H}), 3.39(1 / 2 \mathrm{AB}, J=13.7,1 \mathrm{H}), 2.67(1 / 2 \mathrm{AB}, J=14.0 \mathrm{~Hz}, 1 \mathrm{H}) ;{ }^{13} \mathrm{C} \mathrm{NMR}(75$ $\left.\mathrm{MHz}, \mathrm{CDCl}_{3}\right) \delta 171.6,170.6,137.9,136.4,136.0,135.8,133.3,130.6,129.9,128.4$, $128.0,126.9,126.8,125.7,117.8,112.5,66.5,52.9,50.6,39.8$; IR (thin film) $v 2949$, 1739, 1638, 1410, $1242 \mathrm{~cm}^{-1}$; MS m/z (\%) 373 (20), 342 (8), 314 (8), 282 (25), 105 (100); EI-HRMS calcd for $\mathrm{C}_{24} \mathrm{H}_{23} \mathrm{NO}_{3} \mathrm{~m} / \mathrm{z}\left[\mathrm{M}^{+}\right] 373.1678$, found 373.1664 .
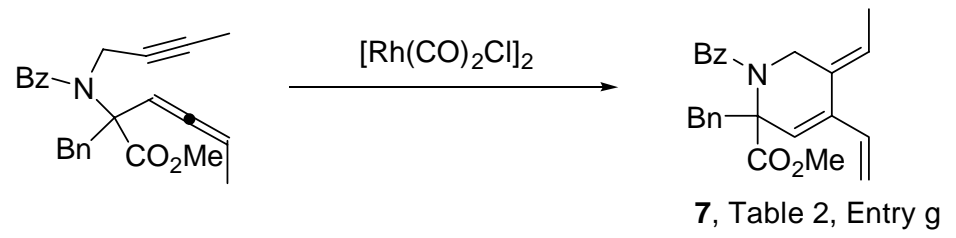

1-Benzoyl-2-benzyl-5-ethylidene-4-vinyl-1,2,5,6-tetrahydropyridine-2-carboxylic acid methyl ester (7, Table 2, Entry g). Followed general procedure for the rhodium catalyzed formal Alder-ene reaction (page s23): 
2-(Benzoyl-but-2-ynyl-amino)-2-benzylhexa-3,4-dienoic acid methyl ester (20 mg, 0.052 $\mathrm{mmol}),\left[\mathrm{Rh}(\mathrm{CO})_{2} \mathrm{Cl}\right]_{2}(1.0 \mathrm{mg}, 0.002 \mathrm{mmol})$

Yield (16 mg, 80\%).

${ }^{1} \mathrm{H}$ NMR $\left(300 \mathrm{MHz}, \mathrm{CDCl}_{3}\right) \delta$ 7.47-7.39 (m, 5H), 7.31-7.28 (m, 5H), 6.36 (dd, $J=17.2$, $10.7 \mathrm{~Hz}, 1 \mathrm{H}), 5.75$ (s, 1H), 5.63 (q, $J=7.0 \mathrm{~Hz}, 1 \mathrm{H}), 5.50$ (dd, $J=17.2,1.5 \mathrm{~Hz}, 1 \mathrm{H}), 5.27$ (dd, $J=10.7,1.5 \mathrm{~Hz}, 1 \mathrm{H}), 4.13(1 / 2 \mathrm{AB}, J=13.6 \mathrm{~Hz}, 1 \mathrm{H}), 4.13(1 / 2 \mathrm{AB}, J=14.6 \mathrm{~Hz}$, $1 \mathrm{H}), 3.80(\mathrm{~s}, 3 \mathrm{H}), 3.41(1 / 2 \mathrm{AB}, J=13.6 \mathrm{~Hz}, 1 \mathrm{H}), 2.46(1 / 2 \mathrm{AB}, J=14.3 \mathrm{~Hz}, 1 \mathrm{H}), 1.42$ $(\mathrm{d}, J=7.1 \mathrm{~Hz}, 3 \mathrm{H}) ;{ }^{13} \mathrm{C} \mathrm{NMR}\left(75 \mathrm{MHz}, \mathrm{CDCl}_{3}\right) \delta 172.3,171.3,139.5,137.0,136.7$, 134.6, 131.0, 130.1, 129.5, 128.8, 128.3, 127.2, 127.0, 123.5, 123.0, 117.9, 114.7, 67.0, $53.1,44.7,40.3,13.4$; IR (thin film) v 2949, 1738, 1640, $1415 \mathrm{~cm}^{-1}$; MS m/z (\%) 387 (8), 356 (7), 296 (25), 105 (100); EI-HRMS calcd for $\mathrm{C}_{18} \mathrm{H}_{18} \mathrm{NO}_{3} \mathrm{~m} / \mathrm{z}$ [M-91 $\left.{ }^{+}\right]$296.1287; found 296.1283 .

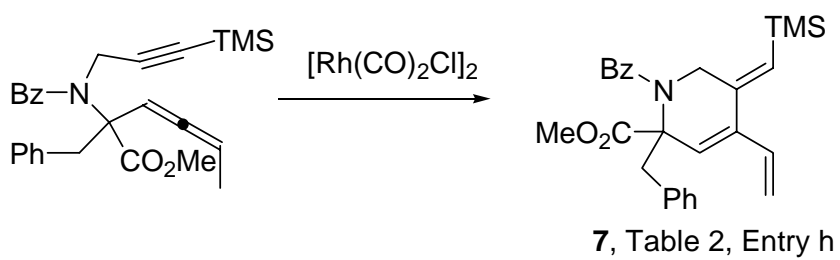

\section{1-Benzoyl-2-benzyl-5-trimethylsilylmethylene-4-vinyl-1,2,5,6-tetrahydropyridine-2-}

carboxylic acid methyl ester (7, Table 2, Entry h). Followed general procedure for the rhodium catalyzed formal Alder-ene reaction (page s23):

2-[Benzoyl-(3-trimethylsilylprop-2-ynyl)-amino]-2-benzylhexa-3,4-dienoic acid methyl ester (46 mg, $0.10 \mathrm{mmol}),\left[\mathrm{Rh}(\mathrm{CO})_{2} \mathrm{Cl}\right]_{2}(2 \mathrm{mg}, 0.005 \mathrm{mmol})$.

Yield (42 mg, 92\%).

${ }^{1} \mathrm{H}$ NMR (300 MHz, $\left.\mathrm{CDCl}_{3}\right) \delta$ 7.38-7.18 (m, 10H), $6.32(\mathrm{dd}, J=17.3,10.8 \mathrm{~Hz} 1 \mathrm{H}), 5.82$ (s, 1H), $5.52(\mathrm{~s}, 1 \mathrm{H}), 5.43(\mathrm{dd}, J=17.3,1.5 \mathrm{~Hz} 1 \mathrm{H}), 5.21$ (dd, $J=10.8,1.5 \mathrm{~Hz} 1 \mathrm{H}), 4.06$ $(1 / 2 \mathrm{AB}, J=13.7 \mathrm{~Hz}, 1 \mathrm{H}), 4.00(1 / 2 \mathrm{AB}, J=14.1 \mathrm{~Hz}, 1 \mathrm{H}), 3.75(\mathrm{~s}, 3 \mathrm{H}), 3.34(1 / 2 \mathrm{AB}, J$ $=13.7 \mathrm{~Hz}, 1 \mathrm{H}) 2.70(1 / 2 \mathrm{AB}, J=14.1,1.21 \mathrm{~Hz}, 1 \mathrm{H}),-0.35(\mathrm{~s}, 9 \mathrm{H}) ;{ }^{13} \mathrm{C} \mathrm{NMR}(75 \mathrm{MHz}$, $\left.\mathrm{CDCl}_{3}\right) \delta 171.8,170.3,142.5,139.5,136.5,136.3,134.0,130.6,129.7,128.5,128.0$, 126.9, 126.7, 124.9, 117.7, 65.7, 52.8, 49.3, 39.7, -0.7; IR (neat) v 3029, 2951, 1740, 1642, 1408, 1252, $1218 \mathrm{~cm}^{-1}$; MS m/z (\%) 445 (5), 430 (5), 354 (40), 105 (100); EIHRMS calcd for $\mathrm{C}_{27} \mathrm{H}_{31} \mathrm{NO}_{3} \mathrm{Si}\left[\mathrm{M}^{+}\right] \mathrm{m} / \mathrm{z}$ 445.2073, found 445.2076. 


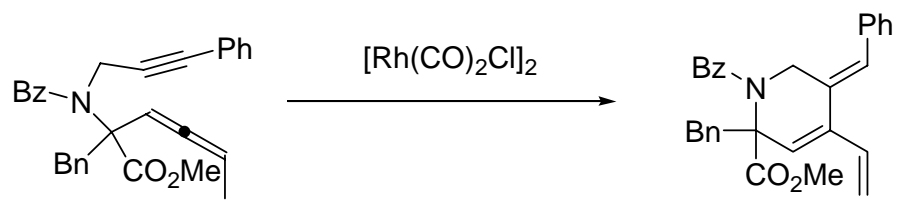

7, Table 2, Entry i

\section{1-Benzoyl-2-benzyl-5-benzylidene-4-vinyl-1,2,5,6-tetrahydropyridine-2-carboxylic}

acid methyl ester (7, Table 2, Entry i). Followed general procedure for the rhodium catalyzed formal Alder-ene reaction (page s23):

2-[Benzoyl-(3-phenylprop-2-ynyl)-amino]-2-benzylhexa-3,4-dienoic acid methyl ester (26 mg, $0.058 \mathrm{mmol}),\left[\mathrm{Rh}(\mathrm{CO})_{2} \mathrm{Cl}\right]_{2}(1 \mathrm{mg}, 0.003 \mathrm{mmol})$

Yield (21 mg, $81 \%)$.

${ }^{1} \mathrm{H}$ NMR $\left(300 \mathrm{MHz}, \mathrm{CDCl}_{3}\right) \delta$ 7.29-7.00 (m, $\left.13 \mathrm{H}\right), 6.82-6.79(\mathrm{~m}, 2 \mathrm{H}), 6.61(\mathrm{~s}, 1 \mathrm{H}), 6.43$ (ddd, $J=17.3,10.7,0.8 \mathrm{~Hz}, 1 \mathrm{H}), 5.89$ (s, 1H), 5.56 (dd, $J=17.3,1.5 \mathrm{~Hz}, 1 \mathrm{H}), 5.33$ (dd, $J$ $=10.7,1.5 \mathrm{~Hz}, 1 \mathrm{H}), 4.41(1 / 2 \mathrm{AB}, J=14.2 \mathrm{~Hz}, 1 \mathrm{H}), 4.10(1 / 2 \mathrm{AB}, J=13.6 \mathrm{~Hz}, 1 \mathrm{H}), 3.77$ (s, 3H), $3.41(1 / 2 \mathrm{AB}, J=13.6 \mathrm{~Hz}, 1 \mathrm{H}), 2.68(1 / 2 \mathrm{AB}, J=14.2,1.4 \mathrm{~Hz}, 1 \mathrm{H}) ;{ }^{13} \mathrm{C} \mathrm{NMR}$ $\left(75 \mathrm{MHz}, \mathrm{CDCl}_{3}\right) \delta 172.1,171.2,139.8,136.9,136.1,135.8,134.5,131.0,130.5,129.7$, $128.8,128.5,128.4,128.0,127.5,127.1,127.0,125.6,118.5,66.9,53.2,45.4$, 40.3; IR (thin film) $\vee 2918,1738,1639,1413,1223 \mathrm{~cm}^{-1}$; MS m/z (\%) 449 (10), 390 (15), 358 (50), 105 (100); EI-HRMS calcd for $\mathrm{C}_{30} \mathrm{H}_{27} \mathrm{NO}_{3} \mathrm{~m} / \mathrm{z}\left[\mathrm{M}^{+}\right]$449.1991; found 449.2011.

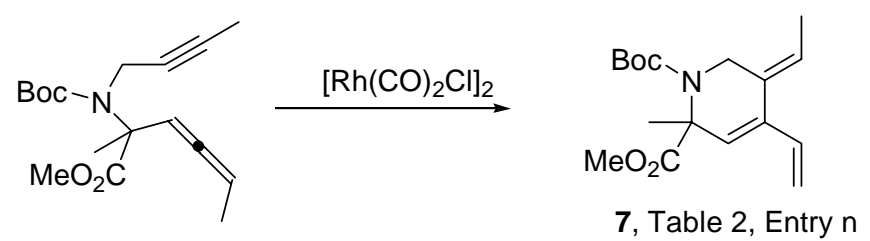

5-Ethylidene-2-methyl-4-vinyl-5,6-dihydro-2H-pyridine-1,2-dicarboxylic acid 1-tertbutyl ester 2-methyl ester (7, Table 2, Entry n). Compound has been previously synthesized in the Curran group via the same general procedure for the allenic Alder-ene reaction (page s23) in $79 \%$ yield. $^{2}$

${ }^{1} \mathrm{H}$ NMR $\left(300 \mathrm{MHz}, \mathrm{CDCl}_{3}\right) \delta 6.31(\mathrm{ddd}, J=17.2,10.7,0.8 \mathrm{~Hz}, 1 \mathrm{H}), 5.70(\mathrm{q}, \mathrm{J}=7.1 \mathrm{~Hz}$, 1H). 5.39 (s, 1H), $5.34(\mathrm{dd}, J=17.2,1.8 \mathrm{~Hz}, 1 \mathrm{H}), 5.12(\mathrm{dd}, J=10.7,1.7 \mathrm{~Hz}, 1 \mathrm{H}), 4.66$ $(1 / 2 \mathrm{AB}, J=14.0 \mathrm{~Hz}, 1 \mathrm{H}), 3.65(\mathrm{~s}, 3 \mathrm{H}), 3.59(1 / 2 \mathrm{AB}, J=14.0 \mathrm{~Hz}, 1 \mathrm{H}), 1.78$ (d, $J=7.1$ $\mathrm{Hz}, 3 \mathrm{H}), 1.55$ (s, 3H), 1.41 (s, 9H); ${ }^{13} \mathrm{C} \mathrm{NMR}\left(75 \mathrm{MHz}, \mathrm{CDCl}_{3}\right) \delta$ 173.3, 154.4, 136.4, 134.4, 124.1, 122.9, 117.3, 81.0, 62.1, 52.4, 39.7, 28.3, 22.4, 13.5; MS m/z (\%) 307 (26), 
248 (47), 192 (100), 148 (56), 57 (60); EI-HRMS calcd for $\mathrm{C}_{17} \mathrm{H}_{25} \mathrm{NO}_{4} \mathrm{~m} / \mathrm{z}\left[\mathrm{M}^{+}\right]$ 307.1784; found 307.1783 .

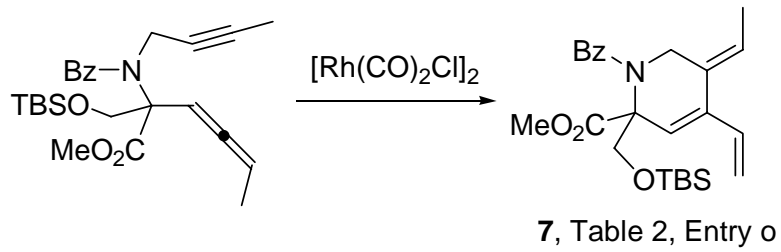

\section{1-Benzoyl-2-(tert-butyldimethylsilyloxymethyl)-5-ethylidene-4-vinyl-1,2,5,6-} tetrahydropyridine-2-carboxylic acid methyl ester (7, Table 2, Entry o). Followed general procedure for the rhodium catalyzed formal Alder-ene reaction (page s23):

2-(Benzoyl-but-2-ynylamino)-2-(tert-butyldimethylsilyloxymethyl)-hexa-3,4-dienoic acid methyl ester (36 mg, $0.082 \mathrm{mmol}),\left[\mathrm{Rh}(\mathrm{CO})_{2} \mathrm{Cl}\right]_{2}(1.5 \mathrm{mg}, 0.0039 \mathrm{mmol})$. Yield (32 mg, $89 \%$ ).

${ }^{1} \mathrm{H}$ NMR $\left(300 \mathrm{MHz}, \mathrm{CDCl}_{3}\right) \delta(7.47-7.40(\mathrm{~m}, 5 \mathrm{H}), 6.38(\mathrm{dd}, J=17.2,10.7 \mathrm{~Hz}, 1 \mathrm{H}), 5.75$ (q, $J=7.0 \mathrm{~Hz}, 1 \mathrm{H}), 5.57$ (s, 1H), 5.44 (dd, $J=17.2,1.5 \mathrm{~Hz}, 1 \mathrm{H}$ ), 5.21 (dd, $J=10.7,1.5$ $\mathrm{Hz}, 1 \mathrm{H}), 4.70(1 / 2 \mathrm{AB}, J=10.2 \mathrm{~Hz}, 1 \mathrm{H}), 4.51(1 / 2 \mathrm{AB}, J=14.1 \mathrm{~Hz}, 1 \mathrm{H}), 4.19(1 / 2 \mathrm{AB}, J$ $=10.2 \mathrm{~Hz}, 1 \mathrm{H}), 3.99(1 / 2 \mathrm{AB}, J=14.0 \mathrm{~Hz}, 1 \mathrm{H}), 3.69(\mathrm{~s}, 3 \mathrm{H}), 1.58(\mathrm{~d}, J=7.1 \mathrm{~Hz}, 1 \mathrm{H})$, 0.85 (s, 9H), 0.06 (s, 3H), 0.05 (s, 3H); ${ }^{13} \mathrm{C}$ NMR (75 MHz, $\left.\mathrm{CDCl}_{3}\right) \delta 170.9,170.8$, 139.6, 136.2, 134.4, 129.9, 129.7, 128.5, 127.0, 122.6, 122.4, 117.5, 66.3, 65.4, 52.5, 45.4, 25.8, 18.1, 13.4, -5.4, -5.5; IR (thin film) v 2954, 2929, 2037, 1740, $1644 \mathrm{~cm}^{-1}$; MS m/z (\%) 441 (23), 411 (63), 384 (66), 296 (100); EI-HRMS calcd for $\mathrm{C}_{25} \mathrm{H}_{35} \mathrm{NO}_{4} \mathrm{Si} \mathrm{m} / \mathrm{z}$ $\left[\mathrm{M}^{+}\right]$441.2335; found 441.2333 . 
General procedure for rhodium catalyzed Pauson-Khand reaction for the preparation of $[6,5]$ P-K products.
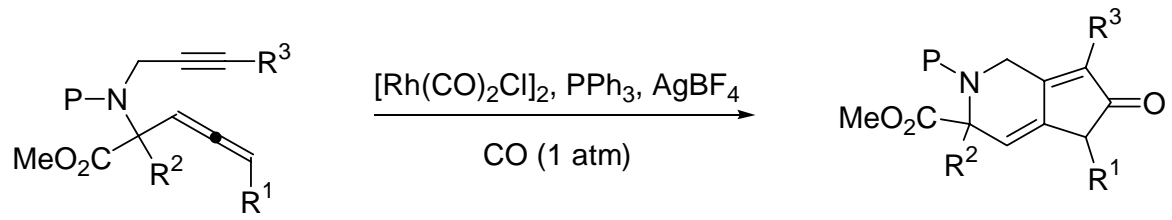

To a flame dried test tube equipped with a magnetic stirring bar was added $\left[\mathrm{Rh}(\mathrm{CO})_{2} \mathrm{Cl}\right]_{2}$ (0.05 mmol) and DCE $(1 \mathrm{~mL})$. To this solution, $\mathrm{PPh}_{3}(0.15 \mathrm{mmol})$ in DCE $(1 \mathrm{~mL})$ was added dropwise via a syringe at room temperature. The test tube was evacuated under vacuum by inserting a needle and charged with $\mathrm{CO}$ from a balloon 3 times. After 5 min $\mathrm{AgBF}_{4}(0.1 \mathrm{mmol}$ of a $0.05 \mathrm{M}$ or $0.1 \mathrm{M}$ solution in DCE) was added dropwise via a syringe. The mixture was stirred for an additional 5-10 min at room temperature and then the allenyne $(0.5 \mathrm{mmol})$ in DCE $(2 \mathrm{~mL})$ was added via a syringe. The reaction was followed by TLC and upon completion (1-2 $\mathrm{h}$ at room temperature or $40{ }^{\circ} \mathrm{C}$ in some cases) it was directly applied to a silica gel column. Gradient elution (hexanes-EtOAc, 9 : 1 to $3: 1, \mathrm{v} / \mathrm{v}$ ) afforded the desired 4-alkylidene cyclopentenones.

Notes: $\left[\mathrm{Rh}(\mathrm{CO})_{2} \mathrm{Cl}\right]_{2}$ and $\mathrm{PPh}_{3}$ were both weighed in an open atmposphere. $\mathrm{AgBF}_{4}$ was weighed in a glove box under $\mathrm{N}_{2}$ atmosphere and caution was taken to limit exposure to light. DCE solution of $\mathrm{AgBF}_{4}$ was prepared by sonication for $5 \mathrm{~min}$.
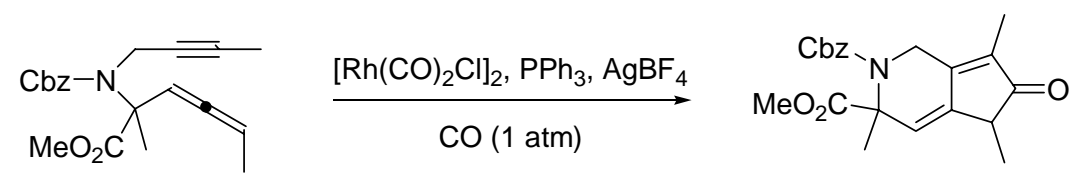

8, Table 3, Entry b

3,5,7-Trimethyl-6-oxo-1,3,5,6-tetrahydro-[2]pyrindine-2,3-dicarboxylic acid 2benzyl ester 3-methyl ester (8, Table 3, Entry b). Followed general procedure for the preparation of $[6,5] \mathrm{P}-\mathrm{K}$ products (page s30):

2-(Benzyloxycarbonyl-but-2-ynyl-amino)-2-methylhexa-3,4-dienoic acid methyl ester (25 mg, $0.07 \mathrm{mmol}),\left[\mathrm{Rh}(\mathrm{CO})_{2} \mathrm{Cl}\right]_{2}(3 \mathrm{mg}, 0.01 \mathrm{mmol}), \mathrm{PPh}_{3}(5 \mathrm{mg}, 0.02 \mathrm{mmol}), \mathrm{AgBF}_{4}$ (150 $\mu \mathrm{L}$ of $0.1 \mathrm{M}$ solution in DCE, $0.02 \mathrm{mmol}$ ).

Yield (19 mg, 75\%).

${ }^{1} \mathrm{H}$ NMR (300 MHz, DMSO-d 6 ) $\delta$ 7.41-7.30 (m, 5H), $5.72(\mathrm{~s}, 1 \mathrm{H}), 5.15$ (s, 2H), $4.86(1 / 2$ $\mathrm{AB}, J=18.6 \mathrm{~Hz}, 1 \mathrm{H}), 4.34(1 / 2 \mathrm{AB}, J=18.0 \mathrm{~Hz}, 1 \mathrm{H}), 3.48$ (br s, 3H), 2.87 (q, $J=7.4$ 
$\mathrm{Hz}, 1 \mathrm{H}), 1.73(\mathrm{~s}, 3 \mathrm{H}), 1.57$ (s, 3H), 1.07 (d, $J=7.5 \mathrm{~Hz}, 3 \mathrm{H}) ;{ }^{13} \mathrm{C}$ NMR $(75 \mathrm{MHz}$, DMSO$\left.d_{6}\right) \delta 206.4,192.6,172.7,155.5,154.9,138.6,137.0,134.6,129.2,128.8,128.5,121.7$, 67.7, 62.5, 53.1, 41.6, 40.7, 22.9, 14.1, 8.5; IR (thin film) v 2916, 1744, 1700, $1234 \mathrm{~cm}^{-1}$; MS m/z (\%) 369 (15), 310 (40), 266 (27), 91 (100); EI-HRMS calcd for $\mathrm{C}_{21} \mathrm{H}_{23} \mathrm{NO}_{5} \mathrm{~m} / \mathrm{z}$ $\left[\mathrm{M}^{+}\right]$369.1576; found 369.1581 .
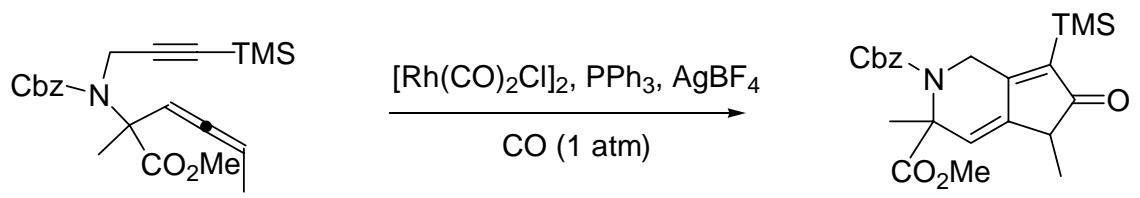

8, Table 3, Entry c

\section{3,5-Dimethyl-6-oxo-7-trimethylsilyl-1,3,5,6-tetrahydro-[2]pyrindine-2,3-dicarboxylic} acid 2-benzyl ester 3-methyl ester (8, Table 3, Entry c). Followed general procedure for the preparation of $[6,5] \mathrm{P}-\mathrm{K}$ products (page s30):

2-[Benzyloxycarbonyl-(3-trimethylsilylprop-2-ynyl)-amino]-2-methylhexa-3,4-dienoic acid methyl ester (30 mg, $0.075 \mathrm{mmol}),\left[\mathrm{Rh}(\mathrm{CO})_{2} \mathrm{Cl}\right]_{2}(3 \mathrm{mg}, 0.01 \mathrm{mmol}), \mathrm{PPh}_{3}(6 \mathrm{mg}$, $0.02 \mathrm{mmol}), \mathrm{AgBF}_{4}(335 \mu \mathrm{L}$ of $0.05 \mathrm{M}$ solution in DCE, $0.02 \mathrm{mmol})$.

Yield (25 mg, 78\%).

${ }^{1} \mathrm{H}$ NMR (300 MHz, DMSO-d $\left.6,323 \mathrm{~K}\right) \delta 7.37$ (s, 5H), $5.80(\mathrm{~s}, 1 \mathrm{H}), 5.18-5.09(\mathrm{~m}, 2 \mathrm{H})$, $5.01(1 / 2 \mathrm{AB}, J=18.6 \mathrm{~Hz}, 1 \mathrm{H}), 4.46(1 / 2 \mathrm{AB}, J=18.6 \mathrm{~Hz}, 1 \mathrm{H}), 3.53(\mathrm{~s}, 3 \mathrm{H}), 2.84(\mathrm{q}, J=$ $7.4 \mathrm{~Hz}, 1 \mathrm{H}), 1.61$ (s, 3H), 1.07 (d, $J=7.4 \mathrm{~Hz}, 3 \mathrm{H}), 0.12(\mathrm{~s}, 9 \mathrm{H}) ;{ }^{13} \mathrm{C} \mathrm{NMR}(75 \mathrm{MHz}$, DMSO- $\left.d_{6}, 323 \mathrm{~K}\right) \delta 209.5,171.4,166.2,154.4,139.7,136.7,135.9,128.1,127.8,127.4$, 122.9, 66.8, 61.3, 52.1, 41.7, 41.6, 21.3, 13.3, -1.3; IR (thin film) v 2953, 1746, 1694, $1248 \mathrm{~cm}^{-1}$; MS m/z (\%) 427 (11), 384 (30), 368 (32), 324 (27), 91 (100); EI-HRMS calcd for $\mathrm{C}_{23} \mathrm{H}_{29} \mathrm{NO}_{5} \mathrm{Si} / 2 / 2\left[\mathrm{M}^{+}\right]$427.1815; found 427.1821.
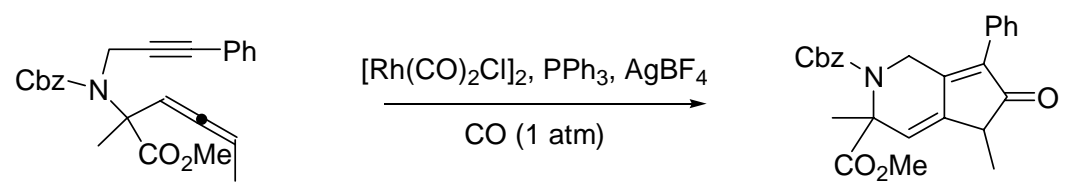

8, Table 3, Entry d

3,5-Dimethyl-6-oxo-7-phenyl-1,3,5,6-tetrahydro-[2]pyrindine-2,3-dicarboxylic acid 2-benzyl ester 3-methyl ester (8, Table 3, Entry d). Followed general procedure for the preparation of $[6,5] \mathrm{P}-\mathrm{K}$ products (page s30): 
2-[Benzyloxycarbonyl-(3-phenylprop-2-ynyl)-amino]-2-methylhexa-3,4-dienoic acid methyl ester (31 mg, $0.08 \mathrm{mmol}),\left[\mathrm{Rh}(\mathrm{CO})_{2} \mathrm{Cl}\right]_{2}(3 \mathrm{mg}, 0.01 \mathrm{mmol}), \mathrm{PPh}_{3}(6 \mathrm{mg}, 0.02$ $\mathrm{mmol}), \mathrm{AgBF}_{4}(340 \mu \mathrm{L}$ of $0.05 \mathrm{M}$ solution in DCE, $0.02 \mathrm{mmol})$.

Yield (25 mg, 75\%).

${ }^{1} \mathrm{H}$ NMR (300 MHz, DMSO-d 6 , $\left.323 \mathrm{~K}\right) \delta$ 7.47-7.32 (m, 10H), 5.90 (s, 1H), 5.16-5.08 (m, 2H), $4.99(1 / 2 \mathrm{AB}, J=18.6 \mathrm{~Hz}, 1 \mathrm{H}), 4.66(1 / 2 \mathrm{AB}, J=18.6 \mathrm{~Hz}, 1 \mathrm{H}), 3.51(\mathrm{~s}, 3 \mathrm{H}), 3.09$ (q, $J=7.4 \mathrm{~Hz}, 1 \mathrm{H}), 1.65(\mathrm{~s}, 3 \mathrm{H}), 1.20(\mathrm{~d}, J=7.4 \mathrm{~Hz}, 3 \mathrm{H}) ;{ }^{13} \mathrm{C}$ NMR (75 MHz, DMSO- $d_{6}$, $323 \mathrm{~K}) \delta 203.7,171.4,154.4,154.1,137.7,135.9$, 134.3, 130.0, 128.4, 128.2, 128.1, 127.7, 127.3, 123.2, 66.7, 61.7, 52.1, 40.2, 21.4, 13.1; IR (thin film) v 2931, 1745, 1703, $1241 \mathrm{~cm}^{-1}$; MS m/z (\%) 431 (10), 372 (86), 328 (45), 91 (100); EI-HRMS calcd for $\mathrm{C}_{26} \mathrm{H}_{25} \mathrm{NO}_{5} \mathrm{~m} / \mathrm{z}\left[\mathrm{M}^{+}\right]$431.1733; found 431.1735.
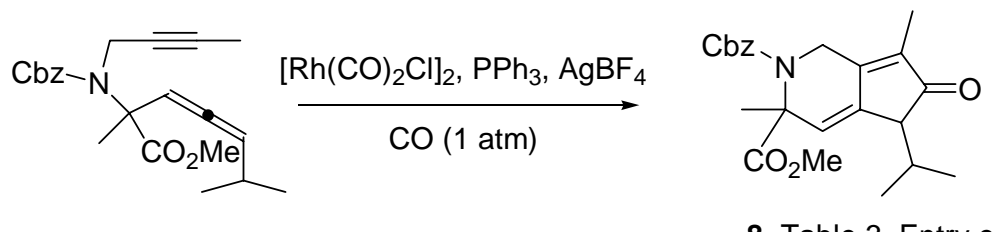

8, Table 3, Entry e

\section{5-Isopropyl-3,7-dimethyl-6-oxo-1,3,5,6-tetrahydro-[2]pyrindine-2,3-dicarboxylic}

acid 2-benzyl ester 3-methyl ester (8, Table 3, Entry e). Followed general procedure for the preparation of $[6,5] \mathrm{P}-\mathrm{K}$ products (page s30):

2-(Benzyloxycarbonylbut-2-ynylamino)-2,6-dimethylhepta-3,4-dienoic acid methyl ester (98 mg, $0.265 \mathrm{mmol}),\left[\mathrm{Rh}(\mathrm{CO})_{2} \mathrm{Cl}\right]_{2}(5 \mathrm{mg}, 0.01 \mathrm{mmol}), \mathrm{PPh}_{3}(10 \mathrm{mg}, 0.04 \mathrm{mmol})$, $\mathrm{AgBF}_{4}(583 \mu \mathrm{L}$ of $0.1 \mathrm{M}$ solution in DCE, $0.0583 \mathrm{mmol})$.

The reaction mixture was heated to $35^{\circ} \mathrm{C}$ for $2 \mathrm{~h}$. Yield (78 $\mathrm{mg}, 74 \%$ ).

${ }^{1} \mathrm{H}$ NMR $\left(300 \mathrm{MHz}, \mathrm{CDCl}_{3}, 320 \mathrm{~K}\right) \delta$ 7.39-7.33 (m, 5H), $5.54(\mathrm{~s}, 1 \mathrm{H}), 5.25(\mathrm{~d}, J=12.2$ $\mathrm{Hz}, 1 \mathrm{H}), 5.17(\mathrm{~d}, J=12.2 \mathrm{~Hz}, 1 \mathrm{H}), 4.95(1 / 2 \mathrm{AB}, J=18.6 \mathrm{~Hz}, 1 \mathrm{H}), 4.21(1 / 2 \mathrm{AB}, J=$ $18.6 \mathrm{~Hz}, 1 \mathrm{H}), 3.56$ (br s, 3H), 2.77-2.76 (m, 1H), 2.30-2.17 (m, 1H), $1.81(\mathrm{~s}, 3 \mathrm{H}), 1.69$ (s, $3 \mathrm{H}), 0.93(\mathrm{~d}, J=7.0 \mathrm{~Hz}, 3 \mathrm{H}), 0.86(\mathrm{~d}, J=6.9 \mathrm{~Hz}, 3 \mathrm{H}) ;{ }^{13} \mathrm{C} \mathrm{NMR}\left(75 \mathrm{MHz}, \mathrm{CDCl}_{3}, 320\right.$ K) $\delta 205.9,172.5,155.4,155.3,136.0,135.9,135.8,128.5,128.3,121.6,67.8,62.3$, $52.5,52.1,40.5,29.8,22.5,19.7,17.9,7.9$; IR (thin film) v 2958, 1745, 1701, $1238 \mathrm{~cm}^{-1}$. MS m/z (\%) 397 (33), 338 (30), 294 (21), 91 (100); EI-HRMS calcd for $\mathrm{C}_{23} \mathrm{H}_{27} \mathrm{NO}_{5} \mathrm{~m} / \mathrm{z}$ $\left[\mathrm{M}^{+}\right]$397.1889; found 397.1888. 


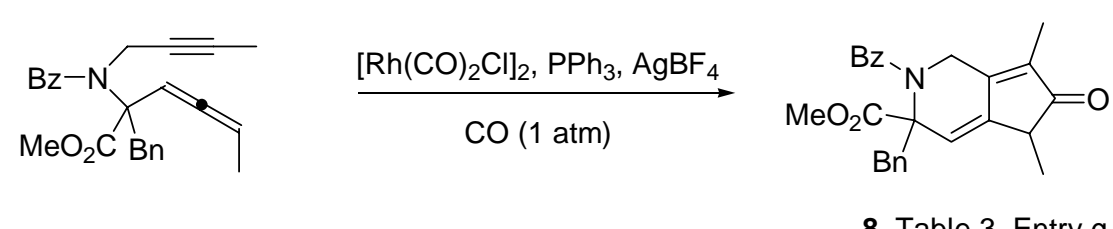

\section{2-benzoyl-3-benzyl-5,7-dimethyl-6-oxo-2,3,5,6-tetrahydro-1H-[2]pyrindine-3-}

carboxylic acid methyl ester (8, Table 3, Entry g). Followed general procedure for the preparation of [6,5] P-K products (page s30):

2-(Benzoyl-but-2-ynyl-amino)-2-benzylhexa-3,4-dienoic acid methyl ester (180 mg, $0.465 \mathrm{mmol}),\left[\mathrm{Rh}(\mathrm{CO})_{2} \mathrm{Cl}\right]_{2}(18 \mathrm{mg}, 0.046 \mathrm{mmol}), \mathrm{PPh}_{3}$ (54 mg, 0.14mmol), $\mathrm{AgBF}_{4}(2$ $\mathrm{mL}$ of $0.05 \mathrm{M}$ solution in DCE, $0.1 \mathrm{mmol})$.

Yield (142 mg, 73\%). The product was obtained in $1.7: 1$ diastereomeric ratio as determined by integration of resonances in the ${ }^{1} \mathrm{H}$ NMR.

IR (neat) v 3027, 2918, 1740, 1704, 1644, 1384, $1250 \mathrm{~cm}^{-1}$; MS m/z (\%) 415 (15), 384 (15), 324 (31), 105 (100); EI-HRMS calcd for $\mathrm{C}_{26} \mathrm{H}_{25} \mathrm{NO}_{4}\left[\mathrm{M}^{+}\right] \mathrm{m} / \mathrm{z}$ 415.1784, found 415.1802 .

The diastereomers were separated by HPLC (hexanes:EtOAc $4: 1$ ).

Isomer $1\left(\mathrm{R}_{\mathrm{f}}=0.21 \mathrm{TLC}\right.$ hexanes : EtOAc, $\left.2: 1\right):{ }^{1} \mathrm{H} \mathrm{NMR}\left(300 \mathrm{MHz}, \mathrm{CDCl}_{3}\right) \delta 7.46$ $6.98(\mathrm{~m}, 10 \mathrm{H}), 5.71(\mathrm{~s}, 1 \mathrm{H}), 4.34(1 / 2 \mathrm{AB}, J=17.7 \mathrm{~Hz}, 1 \mathrm{H}), 4.15(1 / 2 \mathrm{AB}, J=13.7 \mathrm{~Hz}$ $1 \mathrm{H}), 3.79$ (s, 3H), $3.38(1 / 2 \mathrm{AB}, J=13.7 \mathrm{~Hz} 1 \mathrm{H}), 2.86(1 / 2 \mathrm{AB}, J=17.7 \mathrm{~Hz}, 1 \mathrm{H}), 2.84$ $(\mathrm{q}, J=7.4 \mathrm{~Hz}, 1 \mathrm{H}), 1.46(\mathrm{~s}, 3 \mathrm{H}), 1.25(\mathrm{~d}, J=7.4 \mathrm{~Hz}, 3 \mathrm{H}) ;{ }^{13} \mathrm{C} \mathrm{NMR}\left(75 \mathrm{MHz}, \mathrm{CDCl}_{3}\right) \delta$ 206.5, 171.8, 171.6, 153.6, 140.8, 136.4, 135.9, 134.4, 130.6, 130.2, 128.9, 128.4, 127.4, $126.7,119.9,66.3,53.2,44.4,42.0,40.0,13.6,8.1$;

Isomer $2\left(\mathrm{R}_{\mathrm{f}}=0.16 \mathrm{TLC}\right.$ hexanes : EtOAc, $\left.2: 1\right):{ }^{1} \mathrm{H} \mathrm{NMR}\left(300 \mathrm{MHz}, \mathrm{CDCl}_{3}\right) \delta 7.47$ $6.89(\mathrm{~m}, 10 \mathrm{H}), 5.72(\mathrm{~s}, 1 \mathrm{H}), 4.34(1 / 2 \mathrm{AB}, J=18.2 \mathrm{~Hz}, 1 \mathrm{H}), 4.16(1 / 2 \mathrm{AB}, J=13.8 \mathrm{~Hz}$ $1 \mathrm{H}), 3.81$ (s, 3H), 3.39 (1/2 AB, $J=13.8 \mathrm{~Hz} 1 \mathrm{H}), 2.99(1 / 2 \mathrm{AB}, J=18.2 \mathrm{~Hz}, 1 \mathrm{H}), 2.86$ $(\mathrm{q}, J=7.6 \mathrm{~Hz}, 1 \mathrm{H}), 1.44(\mathrm{~s}, 3 \mathrm{H}), 1.31$ (d, $J=7.6 \mathrm{~Hz}, 3 \mathrm{H}) ;{ }^{13} \mathrm{C} \mathrm{NMR}\left(75 \mathrm{MHz}, \mathrm{CDCl}_{3}\right) \delta$ 206.6, 172.1, 171.9, 154.0, 140.5, 136.5, 134.5, 130.9, 130.3, 129.1, 128.4, 127.5, 126.8, 120.2, 66.3, 53.3, 44.9, 41.9, 40.2, 14.5, 8.1.
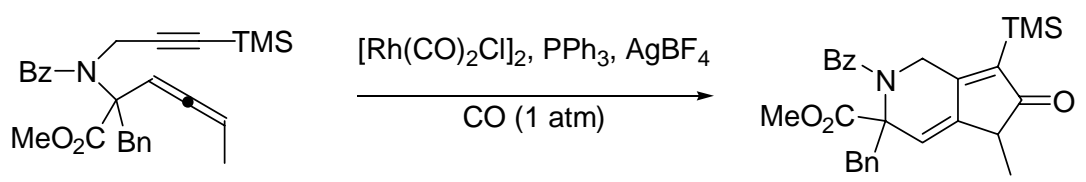

8, Table 3, Entry h 


\section{2-Benzoyl-3-benzyl-5-methyl-6-oxo-7-trimethylsilyl-2,3,5,6-tetrahydro-1H-}

[2]pyrindine-3-carboxylic acid methyl ester (8, Table 3, Entry h). Followed general procedure for the preparation of [6,5] P-K products (page s30):

2-[Benzoyl-(3-trimethylsilylprop-2-ynyl)-amino]-2-benzylhexa-3,4-dienoic acid methyl ester (50 mg, $0.11 \mathrm{mmol}),\left[\mathrm{Rh}(\mathrm{CO})_{2} \mathrm{Cl}\right]_{2}(4 \mathrm{mg}, 0.01 \mathrm{mmol}), \mathrm{PPh}_{3}(9 \mathrm{mg}, 0.03 \mathrm{mmol})$, $\mathrm{AgBF}_{4}(250 \mu \mathrm{L}$ of $0.1 \mathrm{M}$ solution in DCE, $0.0250 \mathrm{mmol})$. The reaction was heated at 35 ${ }^{\circ} \mathrm{C}$ for $1 \mathrm{~h}$.

Yield (52 mg, 98\%) as a mixture of diastereomers in ratio $1.5: 1$. The diastereomers were separated by semi-preparative HPLC (hexanes-EtOAc, $4: 1 \mathrm{v} / \mathrm{v}$ ).

(major diastereomer - eluting first ) ${ }^{1} \mathrm{H}$ NMR $\left(300 \mathrm{MHz}, \mathrm{CDCl}_{3}\right) \delta$ 7.45-7.19 (m, 10H), $5.78(\mathrm{~s}, 1 \mathrm{H}), 4.41(1 / 2 \mathrm{AB}, J=18.1 \mathrm{~Hz}, 1 \mathrm{H}), 4.15(1 / 2 \mathrm{AB}, J=13.7 \mathrm{~Hz}, 1 \mathrm{H}), 3.82$ (s, $3 \mathrm{H}), 3.38(1 / 2 \mathrm{AB}, J=13.7 \mathrm{~Hz}, 1 \mathrm{H}), 2.86-2.79(\mathrm{~m}, 1 \mathrm{H}), 2.82(1 / 2 \mathrm{AB}, J=16.8 \mathrm{~Hz}, 1 \mathrm{H})$, $1.23(\mathrm{~d}, J=7.4 \mathrm{~Hz}, 1 \mathrm{H}),-0.14(\mathrm{~s}, 9 \mathrm{H}) ;{ }^{13} \mathrm{C} \mathrm{NMR}\left(75 \mathrm{MHz}, \mathrm{CDCl}_{3}\right) \delta 210.2,171.7,171.6$, $165.5,142.8,138.2$, 136.3, 136.0, 130.6, 130.0, 128.9, 128.4, 127.3, 126.3, 121.5, 65.7, 53.2, 46.4, 42.9, 40.0, 13.8, -1.3; IR (thin film) $v 2952,1742,1694,1647,1250 \mathrm{~cm}^{-1}$; MS m/z (\%) 473 (5), 458 (22), 382 (45), 105 (100); EI-HRMS calcd for $\mathrm{C}_{28} \mathrm{H}_{31} \mathrm{NO}_{4} \mathrm{Si} \mathrm{m} / \mathrm{z}$ $\left[\mathrm{M}^{+}\right]$473.2022; found 473.2033.

(minor diastereomer - eluting second ) ${ }^{1} \mathrm{H}$ NMR $\left(300 \mathrm{MHz}, \mathrm{CDCl}_{3}\right) \delta 7.45-7.42(\mathrm{~m}, 3 \mathrm{H})$, 7.34-7.31 (m, 2H), 7.27-7.24 (m, 3H), 7.19-7.16 (m, 2H), 5.77 (d, J=1.0 Hz, 1H), 4.39 $(1 / 2 \mathrm{AB}, J=18.5,1.2 \mathrm{~Hz}, 1 \mathrm{H}), 4.15(1 / 2 \mathrm{AB}, J=13.7 \mathrm{~Hz}, 1 \mathrm{H}), 3.83(\mathrm{~s}, 3 \mathrm{H}), 3.38(1 / 2$ $\mathrm{AB}, J=13.7 \mathrm{~Hz}, 1 \mathrm{H}), 2.94(1 / 2 \mathrm{AB}, J=18.5 \mathrm{~Hz}, 1 \mathrm{H}), 2.80$ (q, $J=7.6 \mathrm{~Hz}, 1 \mathrm{H}), 1.29$ (d, $J$ $=7.5 \mathrm{~Hz}, 3 \mathrm{H}),-0.15(\mathrm{~s}, 9 \mathrm{H}) ;{ }^{3} \mathrm{C} \mathrm{NMR}\left(75 \mathrm{MHz}, \mathrm{CDCl}_{3}\right) \delta 210.0,171.7,171.5,165.8$, 142.2 , 138.0, 136.0, 130.7, 129.9, 128.8, 128.1, 127.2, 126.2, 121.5, 65.5, 53.0, 46.7, 42.5, 39.9, 14.2, -1.5; IR (thin film) v 2953, 1742, 1695, 1649, $1250 \mathrm{~cm}^{-1}$; MS m/z (\%) 473 (5), 458 (22), 382 (45), 105 (100); EI-HRMS calcd for $\mathrm{C}_{28} \mathrm{H}_{31} \mathrm{NO}_{4} \mathrm{Si} \mathrm{m} / \mathrm{z}\left[\mathrm{M}^{+}\right]$ 473.2022; found 473.2042 .
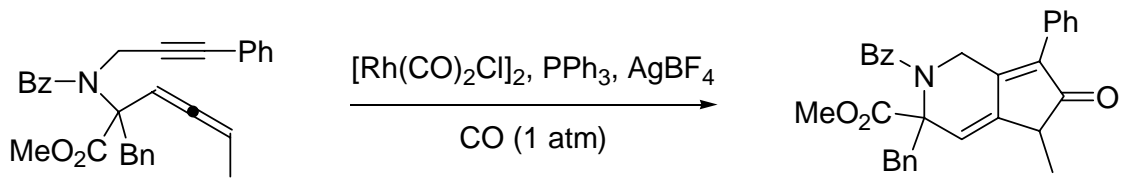

8, Table 3, Entry i 


\section{2-Benzoyl-3-benzyl-5-methyl-6-oxo-7-phenyl-2,3,5,6-tetrahydro-1H-[2]pyrindine-3-}

carboxylic acid methyl ester (8, Table 3, Entry i). Followed general procedure for the preparation of $[6,5] \mathrm{P}-\mathrm{K}$ products (page s30):

2-[Benzoyl-(3-phenylprop-2-ynyl)-amino]-2-benzylhexa-3,4-dienoic acid methyl ester (37 mg, $0.082 \mathrm{mmol}),\left[\mathrm{Rh}(\mathrm{CO})_{2} \mathrm{Cl}\right]_{2}(3 \mathrm{mg}, 0.008 \mathrm{mmol}), \mathrm{PPh}_{3}(6 \mathrm{mg}, 0.02 \mathrm{mmol})$, $\mathrm{AgBF}_{4}(180 \mu \mathrm{L}$ of $0.1 \mathrm{M}$ solution in DCE, $0.0181 \mathrm{mmol})$.

Yield (29 mg, 74\%). Diastereomer ratio $1.65: 1$. The diastereomers were separated by semi-preparative HPLC (hexanes-EtOAc, $4: 1 \mathrm{v} / \mathrm{v}$ )

(major diastereomer $\mathrm{R}_{\mathrm{t}}=15$ min HPLC, hexanes : EtOAc $\left.5: 1, \mathrm{v} / \mathrm{v}\right)$ : ${ }^{1} \mathrm{H}$ NMR (300 $\left.\mathrm{MHz} \mathrm{CDCl}_{3}\right) \delta$ 7.42-7.17 (m, 13H), 7.04-7.00 (m, 2H), $5.88(\mathrm{~d}, J=1.1 \mathrm{~Hz}, 1 \mathrm{H}), 4.60$ $(1 / 2 \mathrm{AB}, J=17.7 \mathrm{~Hz}, 1 \mathrm{H}), 4.18(1 / 2 \mathrm{AB}, J=13.7 \mathrm{~Hz}, 1 \mathrm{H}), 3.81(\mathrm{~s}, 3 \mathrm{H}), 3.44(1 / 2 \mathrm{AB}, J$ $=13.8 \mathrm{~Hz}, 1 \mathrm{H}), 3.10-2.98(\mathrm{~m}, 2 \mathrm{H}), 1.36(\mathrm{~d}, J=7.5 \mathrm{~Hz}, 3 \mathrm{H}) ;{ }^{13} \mathrm{C} \mathrm{NMR}\left(75 \mathrm{MHz}, \mathrm{CDCl}_{3}\right)$ $\delta 204.7,171.8,171.7,153.6,141.2,136.6,135.9,135.5,130.8,130.3,130.1,128.9$, $128.8,128.7,128.7,128.6,127.6,126.8,122.2$, 66.6, 59.9, 53.3, 45.2, 42.9, 40.3, 13.9; IR (thin film) v 2949, 1741, 1706, $16431259 \mathrm{~cm}^{-1}$; MS m/z (\%) 477 (6), 418 (63), 386 (55), 105 (100); EI-HRMS calcd for $\mathrm{C}_{31} \mathrm{H}_{27} \mathrm{NO}_{4} \mathrm{~m} / \mathrm{z}\left[\mathrm{M}^{+}\right]$477.1940; found 477.1954.

(minor diastereomer $\mathrm{R}_{\mathrm{t}}=18$ min HPLC, hexanes : EtOAc $\left.5: 1\right)$ : ${ }^{1} \mathrm{H}$ NMR $(300 \mathrm{MHz}$, $\left.\mathrm{CDCl}_{3}\right) \delta$ 7.40-7.20 (m, 13H), 7.03-6.99 (m, 2H), $5.90(\mathrm{~d}, J=0.9 \mathrm{~Hz}, 1 \mathrm{H}), 4.59(1 / 2 \mathrm{AB}$, $J=18.2,1.2 \mathrm{~Hz}, 1 \mathrm{H}), 4.19(1 / 2 \mathrm{AB}, J=13.8 \mathrm{~Hz}, 1 \mathrm{H}), 3.83(\mathrm{~s}, 3 \mathrm{H}), 3.45(1 / 2 \mathrm{AB}, J=$ $13.8 \mathrm{~Hz}, 1 \mathrm{H}), 3.17(1 / 2 \mathrm{AB}, J=18.3 \mathrm{~Hz}, 1 \mathrm{H}), 3.03$ (q, $J=7.5 \mathrm{~Hz}, 1 \mathrm{H}), 1.41(\mathrm{~d}, J=7.6$ $\mathrm{Hz}, 3 \mathrm{H}) ;{ }^{13} \mathrm{C}$ NMR $\left(75 \mathrm{MHz}, \mathrm{CDCl}_{3}\right) \delta 204.9,172.1,171.7,153.8,140.7,136.4,136.0$, $135.5,130.9,130.3,130.1,129.1,128.8,128.7,128.6,128.5,127.6,126.8,122.5,66.4$, 53.3, 45.6, 42.6, 40.3, 14.9 ; IR (thin film) $v 2950,1741,1706,1645,1235 \mathrm{~cm}^{-1}$; MS m/z (\%) 477 (10), 418 (15), 386 (25), 105 (100); EI-HRMS calcd for $\mathrm{C}_{31} \mathrm{H}_{27} \mathrm{NO}_{4} \mathrm{~m} / \mathrm{z}\left[\mathrm{M}^{+}\right]$ 477.1940; found 477.1941.
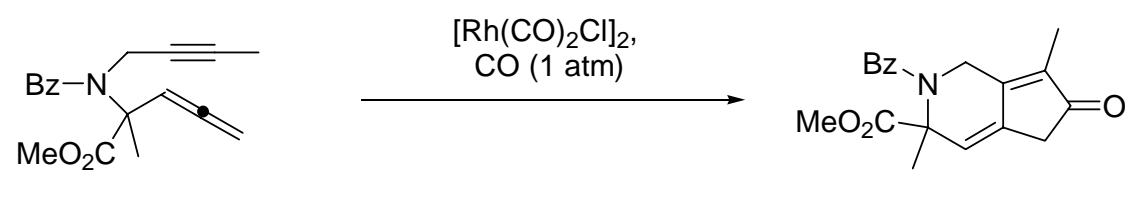

$$
\text { 8, Table 3, Entry I }
$$

2-Benzoyl-3,7-dimethyl-6-oxo-2,3,5,6-tetrahydro-1H-[2]pyrindine-3-carboxylic acid methyl ester (8, Table 3, Entry l). $\left[\mathrm{Rh}(\mathrm{CO})_{2} \mathrm{Cl}\right]_{2}(1.5 \mathrm{mg}, 0.0038 \mathrm{mmol})$ was placed in a test tube and DCE $(0.3 \mathrm{~mL})$ was added. The test tube was sealed with a rubber septum, 
the atmosphere evacuated by inserting a needle and replaced with $\mathrm{CO}$ (balloon) three times. 2-(Benzoyl-but-2-ynyl-amino)-2-methyl-penta-3,4-dienoic acid methyl ester (23 $\mathrm{mg}, 0.077 \mathrm{mmol})$ in DCE $(0.7 \mathrm{~mL})$ was added via syringe. After $2.5 \mathrm{~h}$ the reaction was complete based upon TLC and the light yellow solution was directly applied to a silica gel column. Elution (hexanes-EtOAc, $4: 1$ to $2: 1$, v/v) afforded the title compound (18 $\mathrm{mg}, 72 \%)$ after solvent removal.

${ }^{1} \mathrm{H}$ NMR (300 MHz, $\left.\mathrm{CDCl}_{3}\right) \delta$ 7.51-7.43 (m, 5H), $5.69(\mathrm{~s}, 1 \mathrm{H}), 4.78(1 / 2 \mathrm{AB}, J=17.6$ Hz, 1H), 4.19 (1/2 AB, J = 17.6, $0.9 \mathrm{~Hz}, 1 \mathrm{H}), 3.74$ (s, 3H), 3.02 (s, 1H), 1.79 (s, 3H), $1.69(\mathrm{~s}, 3 \mathrm{H}) ;{ }^{13} \mathrm{C}$ NMR $\left(75 \mathrm{MHz}, \mathrm{CDCl}_{3}\right) \delta 203.5,172.1,171.8,155.3,136.2,135.2$, 133.0, 130.6, 128.8, 127.1, 123.0, 62.4, 52.9, 43.2, 37.4, 20.6, 8.0; IR (thin film) v 2950, 1740, 1704, 1645, $1394 \mathrm{~cm}^{-1}$. MS m/z (\%) 325 (25), 294 (9), 266 (47), 105 (100); EIHRMS calcd for $\mathrm{C}_{19} \mathrm{H}_{19} \mathrm{NO}_{4} \mathrm{~m} / \mathrm{z}\left[\mathrm{M}^{+}\right]$325.1314; found 325.1324 .
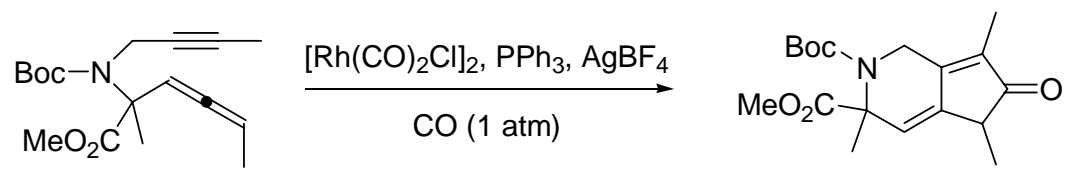

8, Table 3, Entry n

3,5,7-Trimethyl-6-oxo-1,3,5,6-tetrahydro-[2]pyrindine-2,3-dicarboxylic acid 2-tertbutyl ester 3-methyl ester (8, Table 3, Entry n). Followed general procedure for the preparation of $[6,5] \mathrm{P}-\mathrm{K}$ products (page s30):

2-(tert-Butoxycarbonyl-but-2-ynylamino)-2-methylhexa-3,4-dienoic acid methyl ester (50 mg, $0.163 \mathrm{mmol}),\left[\mathrm{Rh}(\mathrm{CO})_{2} \mathrm{Cl}\right]_{2}(6.0 \mathrm{mg}, 0.016 \mathrm{mmol}), \mathrm{PPh}_{3}(13 \mathrm{mg}, 0.049 \mathrm{mmol})$, $\mathrm{AgBF}_{4}(360 \mu \mathrm{L}$ of $0.1 \mathrm{M}$ solution in DCE, $0.0359 \mathrm{mmol})$.

Upon disappearance of the starting material (TLC) the reaction mixture was applied to a small silica gel column and eluted (hexanes-EtOAc, $4: 1 \mathrm{v} / \mathrm{v}$ ) to afford a mixture of CO insertion products ( $40 \mathrm{mg}, 73 \%$ ).

NMR analysis $[6,5]:[5,5]=5: 1$.

The two products were separated by flash chromatography (hexanes-EtOAc, $1: 0$ to $4: 1$ $\mathrm{v} / \mathrm{v})$.

$[5,5]\left(\mathrm{R}_{\mathrm{f}}=0.7\right.$, (hexanes-EtOAc, $\left.\left.3: 1 \mathrm{v} / \mathrm{v}\right)\right)$ spectral data identical to 9 (Table 4, Entry $\left.\mathrm{n}\right)$ formed via the Mo mediated P-K reaction. 
[6,5] $\left(\mathrm{R}_{\mathrm{f}}=0.66\right.$, (hexanes-EtOAc, $\left.\left.3: 1 \mathrm{v} / \mathrm{v}\right)\right){ }^{1} \mathrm{H}$ NMR $\left(300 \mathrm{MHz}, \mathrm{CDCl}_{3}\right) \delta 5.50(\mathrm{~s}, 1 \mathrm{H})$, $4.92(1 / 2 \mathrm{AB}, J=18.6 \mathrm{~Hz}, 1 \mathrm{H}), 4.11(1 / 2 \mathrm{AB}, J=18.8 \mathrm{~Hz}, 1 \mathrm{H}), 3.72$ (s, 3H), 2.83 (q, $J=$ $7.5 \mathrm{~Hz}, 1 \mathrm{H}), 1.84(\mathrm{~s}, 3 \mathrm{H}), 1.66(\mathrm{~s}, 3 \mathrm{H}), 1.48(\mathrm{~s}, 9 \mathrm{H}), 1.21(\mathrm{~d}, J=7.5 \mathrm{~Hz}, 3 \mathrm{H}) ;{ }^{13} \mathrm{C} \mathrm{NMR}$ $\left(75 \mathrm{MHz}, \mathrm{CDCl}_{3}\right) \delta 206.7,173.0,154.8,154.5,138.4,134.7,121.0,81.9,62.0,52.7$, 41.6, 40.1, 28.2, 22.7, 13.7, 8.1; MS m/z (\%) 335 (11), 276 (40), 220 (69), 176 (45), 57 (100); EI-HRMS calcd for $\mathrm{C}_{18} \mathrm{H}_{25} \mathrm{NO}_{5} \mathrm{~m} / \mathrm{z}\left[\mathrm{M}^{+}\right]$335.1733; found 335.1726.
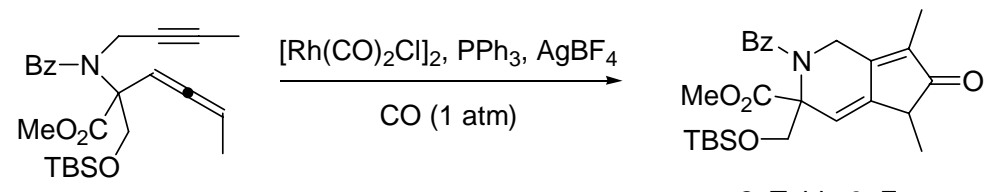

8, Table 3, Entry o

\section{2-Benzoyl-3-(tert-butyl-dimethyl-silyloxymethyl)-5,7-dimethyl-6-oxo-2,3,5,6-} tetrahydro-1H-[2]pyrindine-3-carboxylic acid methyl ester (8, Table 3, Entry o).

Followed general procedure for the preparation of [6,5] P-K products (page s30):

2-(Benzoyl-but-2-ynylamino)-2-(tert-butyldimethylsilyloxymethyl)-hexa-3,4-dienoic acid methyl ester $(50 \mathrm{mg}, 0.11 \mathrm{mmol}),\left[\mathrm{Rh}(\mathrm{CO})_{2} \mathrm{Cl}\right]_{2}(4 \mathrm{mg}, 0.01 \mathrm{mmol}), \mathrm{PPh}_{3}(9 \mathrm{mg}$, $0.03 \mathrm{mmol}), \mathrm{AgBF}_{4}(250 \mu \mathrm{L}$ of $0.1 \mathrm{M}$ solution in DCE, $0.0250 \mathrm{mmol})$.

Yield (43 mg, 81\%). Diastereomer ratio $1.78: 1$. The diastereomers were separated by semi-preparative HPLC (hexanes-EtOAc, $6: 1 \mathrm{v} / \mathrm{v}$ )

IR (thin film) $v 2953,1741,1708,1648,1258 \mathrm{~cm}^{-1}$

(major diastereomer $\mathrm{R}_{\mathrm{t}}=17$ min HPLC, EtOAc : Hex $\left.1: 6\right):{ }^{1} \mathrm{H}$ NMR (300 MHz, $\mathrm{CDCl}_{3}$ ) $\delta$ 7.49-7.43 (m, 5H), $5.56(\mathrm{~s}, 1 \mathrm{H}), 4.75(1 / 2 \mathrm{AB}, J=17.1 \mathrm{~Hz}, 1 \mathrm{H}), 4.74(1 / 2 \mathrm{AB}, J=10.4$ $\mathrm{Hz}, 1 \mathrm{H}), 4.45(1 / 2 \mathrm{AB}, J=17.4 \mathrm{~Hz}, 1 \mathrm{H}), 4.15(1 / 2 \mathrm{AB}, J=10.3 \mathrm{~Hz}, 1 \mathrm{H}), 3.74(\mathrm{~s}, 3 \mathrm{H})$, 2.89 (q, $J=7.5 \mathrm{~Hz}, 1 \mathrm{H}), 1.65$ (s, 3H), 1.28 (d, $J=7.5 \mathrm{~Hz}, 3 \mathrm{H}), 0.81$ (s, 9H), 0.07 (s, 3H), $0.02(\mathrm{~s}, 3 \mathrm{H}) ;{ }^{13} \mathrm{C}$ NMR $\left(75 \mathrm{MHz}, \mathrm{CDCl}_{3}\right) \delta 206.5,171.7,170.5,154.3,144.3,141.3$, 135.9, 134.3, 130.0, 128.8, 126.5, 118.8, 66.1, 65.3, 52.6, 45.6, 41.9, 25.7, 18.0, 13.6, 7.9, -5.4, -5.6; MS m/z (\%) 469 (37), 439 (30), 412 (30), 324 (20), 105 (100); EI-HRMS calcd for $\mathrm{C}_{26} \mathrm{H}_{35} \mathrm{NO}_{5} \mathrm{Si} \mathrm{m} / \mathrm{z}\left[\mathrm{M}^{+}\right]$469.2285; found 469.2280.

(minor diastereomer $\mathrm{R}_{\mathrm{t}}=26$ min HPLC, EtOAc : Hex $\left.1: 6\right):{ }^{1} \mathrm{H}$ NMR (300 MHz, $\mathrm{CDCl}_{3}$ ) $\delta$ 7.50-7.42 (m, 5H), 5.59 (s, 1H), $4.74(1 / 2 \mathrm{AB}, J=18.0 \mathrm{~Hz}, 1 \mathrm{H}), 4.72(1 / 2 \mathrm{AB}, J=10.2$ $\mathrm{Hz}, 1 \mathrm{H}), 4.47$ (1/2 AB, $J=17.8 \mathrm{~Hz}, 1 \mathrm{H}), 4.15(1 / 2 \mathrm{AB}, J=10.3 \mathrm{~Hz}, 1 \mathrm{H}), 3.75$ (s, 3H), 2.91 (q, $J=7.6 \mathrm{~Hz}, 1 \mathrm{H}), 1.64$ (s, 3H), 1.27 (d, $J=7.6 \mathrm{~Hz}, 9 \mathrm{H}), 0.80$ (s, 3H), 0.07 (s, 3H), $0.02(\mathrm{~s}, 3 \mathrm{H}) ;{ }^{13} \mathrm{C}$ NMR $\left(75 \mathrm{MHz}, \mathrm{CDCl}_{3}\right) \delta 206.6,171.9,170.4,154.6,141.2,135.9$, 
$134.2,130.0,128.9,126.5,118.8,65.9,65.1,52.6,45.9,41.6,25.6,17.9,14.2,7.9,-5.5$, 5.7; MS m/z (\%) 469 (7), 439 (30), 412 (35), 324 (22), 105 (100); EI-HRMS calcd for $\mathrm{C}_{26} \mathrm{H}_{35} \mathrm{NO}_{5} \mathrm{Si} \mathrm{m} / \mathrm{z}\left[\mathrm{M}^{+}\right]$469.2285; found 469.2299.

General procedure for the molybdenum mediated allenic Pauson-Khand reaction for the formation of $[5,5]$ P-K products.
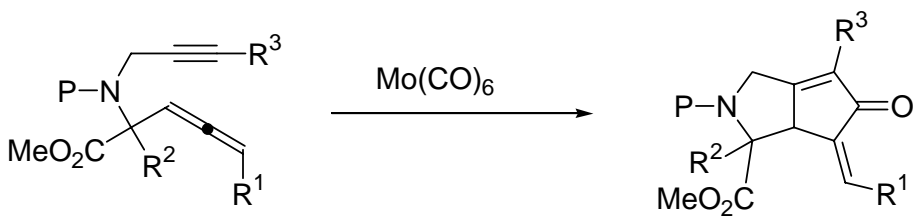

To a solution of the allenyne $(0.3 \mathrm{mmol})$ in toluene $(3 \mathrm{~mL})$ in a $10 \mathrm{~mL}$ round-bottomed flask was added DMSO (3 mmol) followed by $\mathrm{Mo}(\mathrm{CO})_{6}(0.375 \mathrm{mmol})$ at room temperature under $\mathrm{N}_{2}$ atmosphere. The flask was equipped with a reflux condenser and heated slowly to $80-90{ }^{\circ} \mathrm{C}$ in an oil bath. Stirring was continued at this temperature until TLC analysis showed absence of starting material. Upon completion the reaction mixture was directly applied on a small plug of silica gel and eluted (hexanes-EtOAc, $3: 1$ to 1 : $1, \mathrm{v} / \mathrm{v})$. The collected fractions were combined and concentrated under vacuum, and NMR (or HPLC analysis) was performed to determine the product distribution.

Purification of the mixtures was performed by flash chromatography (gradient elution, hexanes-EtOAc v/v).
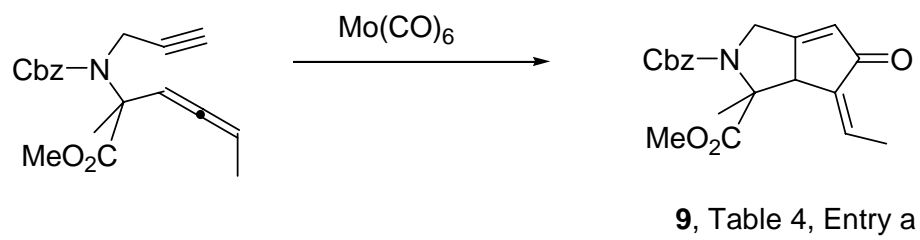

\section{6-Ethylidene-1-methyl-5-oxo-3,5,6,6a-tetrahydro-1H-cyclopenta[c]pyrrole-1,2-}

dicarboxylic acid 2-benzyl ester 1-methyl ester (9, Table 4, Entry a). Followed general procedure for the molybdenum mediated allenic Pauson-Khand reaction for the formation of [5,5] $\mathrm{P}-\mathrm{K}$ products (page s38):

2-(Benzyloxycarbonylprop-2-ynylamino)-2-methylhexa-3,4-dienoic acid methyl ester (80 mg, $0.25 \mathrm{mmol})$, DMSO (174 $\mu \mathrm{L}, 2.45 \mathrm{mmol}), \mathrm{Mo}(\mathrm{CO})_{6}(81 \mathrm{mg}, 0.31 \mathrm{mmol})$. Heated to $80^{\circ} \mathrm{C}$ for $30 \mathrm{~min}$. 
Elution (hexanes-EtOAc, $1: 1 \mathrm{v} / \mathrm{v}$ ) on a small silica gel plug afforded a mixture of compounds ( $82 \mathrm{mg},>95 \%$ ). Product ratios determined by integration of the peaks in the HPLC trace (hexanes-EtOAc, $4: 1 \mathrm{v} / \mathrm{v}$ ): [5,5] Z / E = 7.6 : 1. Purification by flash chromatography (hexanes-EtOAc, $19: 1$ to $2: 1 \mathrm{v} / \mathrm{v}$ ) afforded the major $\mathrm{Z}$ isomer (48 mg. 55\%).

(Z) : $\mathrm{R}_{\mathrm{f}}=0.4$ (TLC, hexanes-EtOAc, $\left.4: 1 \mathrm{v} / \mathrm{v}\right){ }^{1} \mathrm{H}$ NMR (300 MHz, DMSO- $\left.d_{6} 353 \mathrm{~K}\right) \delta$ 7.40-7.32 (m, 5H), 6.29 (s, 1H), 5.98 (q, $J=7.24 \mathrm{~Hz}, 1 \mathrm{H}), 5.10$ (s, 2H), 4.49 (1/2 AB, $J=$ $16.2 \mathrm{~Hz}, 1 \mathrm{H}), 4.37(1 / 2 \mathrm{AB}, J=16.1 \mathrm{~Hz}, 1 \mathrm{H}), 4.01$ (s, 1H), 3.66 (br s, 3H), 2.17 (d, $J=$ $7.3 \mathrm{~Hz}, 3 \mathrm{H}$ ), 1.10 (s, 3H); IR (thin film) v 2950, 1742, 1699, 1643, $1408 \mathrm{~cm}^{-1}$; MS m/z (\%) 355 (10), 296 (18), 252 (19), 91 (100); EI-HRMS calcd for $\mathrm{C}_{20} \mathrm{H}_{21} \mathrm{NO}_{5} \mathrm{~m} / \mathrm{z}\left[\mathrm{M}^{+}\right]$ 355.1420; found 355.1427.

${ }^{13} \mathrm{C}$ NMR - Due to isomerization of the sample at $353 \mathrm{~K}$ data was not collected
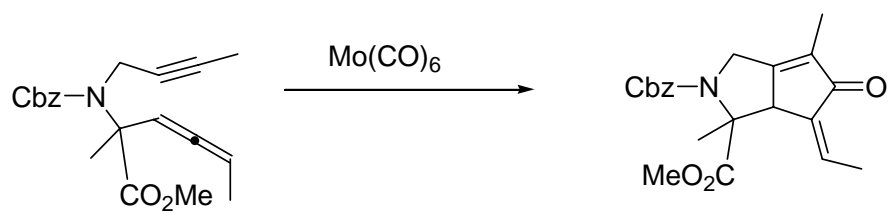

9, Table 4, Entry b

\section{6-Ethylidene-1,4-dimethyl-5-oxo-3,5,6,6a-tetrahydro-1H-cyclopenta[c]pyrrole-1,2-}

dicarboxylic acid 2-benzyl ester 1-methyl ester (9, Table 4, Entry b). Followed general procedure for the molybdenum mediated allenic Pauson-Khand reaction for the formation of [5,5] P-K products (page s38):

2-(Benzyloxycarbonyl-but-2-ynyl-amino)-2-methylhexa-3,4-dienoic acid methyl ester (56 mg, $0.16 \mathrm{mmol})$, DMSO (116 $\mu \mathrm{L}, 1.64 \mathrm{mmol}), \mathrm{Mo}(\mathrm{CO})_{6}(54 \mathrm{mg}, 0.20 \mathrm{mmol})$. Heated to $90^{\circ} \mathrm{C}$ for $45 \mathrm{~min}$.

Flash chromatography by gradient elution (hexanes-EtOAc, $9: 1$ to $4: 1 \mathrm{v} / \mathrm{v}$ ) afforded a mixture of compounds $(60 \mathrm{mg},>95 \%)$. Product ratios determined by NMR: $[5,5]:[6,5]>$ $20: 1 ;[5,5] \mathrm{Z} / \mathrm{E}=7: 1$. The major product was purified by semi-preparative HPLC (hexanes-EtOAc, $4: 1 \mathrm{v} / \mathrm{v}$ ).

(Z) : ${ }^{1} \mathrm{H}$ NMR (300 MHz, DMSO-d 6 353K) $\delta$ 7.38-7.33 (m, 5H), 5.98 (q, $J=7.3 \mathrm{~Hz}, 1 \mathrm{H}$ ), $5.11(\mathrm{~s}, 2 \mathrm{H}), 4.43(1 / 2 \mathrm{AB}, J=16.2 \mathrm{~Hz}, 1 \mathrm{H}), 4.36(1 / 2 \mathrm{AB}, J=16.3 \mathrm{~Hz}, 1 \mathrm{H}), 3.89$ (s, 1H), 3.67 (s, 3H), 2.20 (d, J=7.3 Hz, 3H), 1.75 (s, 3H), 1.06 (s, 3H); ${ }^{13} \mathrm{C}$ NMR (75 MHz, 
$\left.\mathrm{DMSO}_{-} d_{6} 353 \mathrm{~K}\right) \delta 195.9,172.0,160.4,152.7,135.9,135.8,135.5,132.0,127.7,127.3$, 127.0, 66.0, 65.4, 53.7, 51.7, 44.8, 12.9, 12.7, 7.7; IR (thin film) v 2951, 1743, 1698, 1646, $1408 \mathrm{~cm}^{-1}$; MS m/z (\%) 369 (13), 310 (7), 266 (10), 91 (100); EI-HRMS calcd for $\mathrm{C}_{21} \mathrm{H}_{23} \mathrm{NO}_{5} \mathrm{~m} / \mathrm{z}\left[\mathrm{M}^{+}\right] 369.1576$ found 369.1580 .
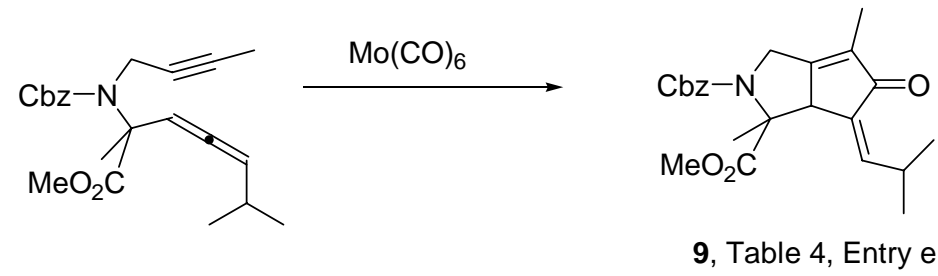

\section{6-Isobutylidene-1,4-dimethyl-5-oxo-3,5,6,6a-tetrahydro-1H-cyclopenta[c]pyrrole-}

1,2-dicarboxylic acid 2-benzyl ester 1-methyl ester (9, Table 4, Entry e). Followed general procedure for the molybdenum mediated allenic Pauson-Khand reaction for the formation of [5,5] P-K products (page s38):

2-(Benzyloxycarbonylbut-2-ynylamino)-2,6-dimethylhepta-3,4-dienoic acid methyl ester (47 mg, $0.13 \mathrm{mmol}$ ), DMSO (90 $\mu \mathrm{L}, 1.27 \mathrm{mmol}$ ), $\mathrm{Mo}(\mathrm{CO})_{6}(42 \mathrm{mg}, 0.16 \mathrm{mmol})$. Heated to $80^{\circ} \mathrm{C}$ for $1 \mathrm{~h}$.

Gradient elution (hexanes-EtOAc, $9: 1$ to $4: 1 \mathrm{v} / \mathrm{v}$ ) on a small silica gel plug afforded a mixture of 2 compounds ( $50 \mathrm{mg},>95 \%$ ). Product ratios determined by NMR: [5,5] Z / E $=6.4: 1$. Purification by flash chromatography afforded the major $\mathrm{Z}$ isomer $(42 \mathrm{mg}$. $83 \%)$.

(Z) : $\mathrm{R}_{\mathrm{f}}=0.52(\mathrm{TLC}$, hexanes-EtOAc, $4: 1 \mathrm{v} / \mathrm{v}){ }^{1} \mathrm{H}$ NMR (300 MHz, DMSO- $\left.d_{6} 353 \mathrm{~K}\right) \delta$ 7.38-7.31 (m, 5H), 5.67 (d, $J=9.8 \mathrm{~Hz}, 1 \mathrm{H}), 5.10(\mathrm{~s}, 2 \mathrm{H}), 4.41(1 / 2 \mathrm{AB}, J=17.5 \mathrm{~Hz}$, $1 \mathrm{H}), 4.35(1 / 2 \mathrm{AB}, J=17.5 \mathrm{~Hz}, 1 \mathrm{H}), 3.99-3.78(\mathrm{~m}, 2 \mathrm{H}), 3.67$ (br s, 3H), 1.76-1.75 (m, $3 \mathrm{H}), 1.06(\mathrm{~s}, 3 \mathrm{H}), 0.99$ (d, $J=5.5 \mathrm{~Hz}, 3 \mathrm{H}), 0.97(\mathrm{~d}, J=5.5 \mathrm{~Hz}, 3 \mathrm{H}) ;{ }^{13} \mathrm{C} \mathrm{NMR}(75 \mathrm{MHz}$, DMSO- $\left._{6} 353 \mathrm{~K}\right) \delta: 195.6,172.2,152.7,147.7,147.4,136.0,129.3,127.9,127.4,127.2$, 65.4, 51.9, 24.7, 21.9, 21.5, 21.2, 8.0; IR (thin film) v 2955, 1744, 1699, 1643, $1407 \mathrm{~cm}^{-1}$; MS m/z (\%) 397 (10), 262 (5), 149 (25), 91 (100); EI-HRMS calcd for $\mathrm{C}_{23} \mathrm{H}_{27} \mathrm{NO}_{5} \mathrm{~m} / \mathrm{z}$ $\left[\mathrm{M}^{+}\right]$397.1889; found 397.1888.

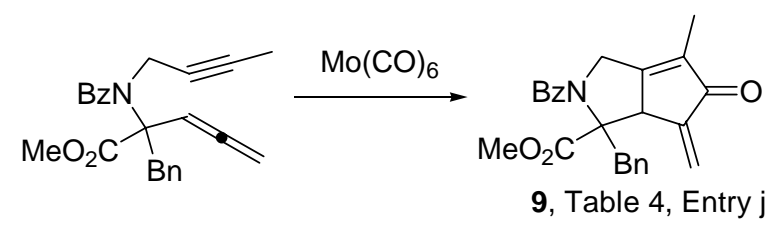




\section{2-Benzoyl-1-benzyl-4-methyl-6-methylene-5-oxo-1,2,3,5,6,6a-hexahydro-}

cyclopenta[c]pyrrole-1-carboxylic acid methyl ester (9, Table 4, Entry j). Followed general procedure for the molybdenum mediated allenic Pauson-Khand reaction for the formation of [5,5] $\mathrm{P}-\mathrm{K}$ products (page s38):

2-(Benzoyl-but-2-ynyl-amino)-2-benzylhexa-3,4-dienoic acid methyl ester (338 mg, $0.906 \mathrm{mmol}), \mathrm{DMSO}(320 \mu \mathrm{L}, 4.53 \mathrm{mmol}), \mathrm{Mo}(\mathrm{CO})_{6}(300 \mathrm{mg}, 1.13 \mathrm{mmol})$. Heated to 90 ${ }^{\circ} \mathrm{C}$ for $45 \mathrm{~min}$. Gradient elution (hexanes-EtOAc, $19: 1$ to $3: 2, \mathrm{v} / \mathrm{v}$ ) gave separation of the major component.

The ratio of products observed by ${ }^{1} \mathrm{H}$ NMR of the crude mixture after filtration on a small silica gel plug is: $[5,5]:[6,5]=5.7: 1$; Diastereomer ratio of $[5,5] 6.4: 1$.

$\mathrm{Rf}=0.3(\mathrm{TLC}$, hexanes-EtOAc, $3: 1 \mathrm{v} / \mathrm{v})$ [5,5] major diastereomer $(210 \mathrm{mg}, 57 \%)$

${ }^{1} \mathrm{H}$ NMR $\left(300 \mathrm{MHz}, \mathrm{CDCl}_{3}\right) \delta$ 7.60-7.28 (m, $\left.10 \mathrm{H}\right), 6.21(\mathrm{~s}, 1 \mathrm{H}), 5.62(\mathrm{~s}, 1 \mathrm{H}), 4.24(1 / 2$ $\mathrm{AB}, J=14.6 \mathrm{~Hz}, 1 \mathrm{H}), 4.19(1 / 2 \mathrm{AB}, J=14.3 \mathrm{~Hz}, 1 \mathrm{H}), 3.99(1 / 2 \mathrm{AB}, J=14.6,1.2 \mathrm{~Hz}$, $1 \mathrm{H}), 3.90(\mathrm{~s}, 1 \mathrm{H}), 3.61(\mathrm{~s}, 3 \mathrm{H}), 3.38(1 / 2 \mathrm{AB}, J=14.3 \mathrm{~Hz}, 1 \mathrm{H}), 1.69(\mathrm{~s}, 3 \mathrm{H}) ;{ }^{13} \mathrm{C} \mathrm{NMR}$ (75 MHz, $\left.\mathrm{CDCl}_{3}\right)$ 195.5, 171.0, 170.2, 162.6, 140.6, 136.1, 135.9, 134.7, 131.3, 131.1, $128.7,128.5,127.5,127.3,117.6,71.0,52.2,50.5,49.5,37.9,8.9$. IR (thin film) v 2950, 1741, 1707, 1644, 1378, $1340 \mathrm{~cm}^{-1}$; MS m/z (\%) 401 (40), 342 (22), 296 (35), 105 (100); EI-HRMS calcd for $\mathrm{C}_{25} \mathrm{H}_{23} \mathrm{NO}_{4} \mathrm{~m} / \mathrm{z}\left[\mathrm{M}^{+}\right] 401.1627$ found 401.1641

$\mathrm{Rf}=0.2(\mathrm{TLC}$, hexanes-EtOAc, $3: 1 \mathrm{v} / \mathrm{v})$ mixture of [5,5] minor diastereomer and [6,5] (82 mg, 22\%).

[5,5]-minor diastereomer : ${ }^{1} \mathrm{H}$ NMR $\left(300 \mathrm{MHz}, \mathrm{CDCl}_{3}\right) \delta$ 7.59-7.44 (m, 5H), 7.17-7.10 (m, 3H), 7.01-6.98 (m, 2H), 6.43 (d, $J=1.9 \mathrm{~Hz}, 1 \mathrm{H}), 5.66(\mathrm{~d}, J=1.3 \mathrm{~Hz}, 1 \mathrm{H}), 4.32$ (d, $J$ $=15.7,1 \mathrm{H}), 4.01(\mathrm{br} \mathrm{s}, 1 \mathrm{H}), 3.94(\mathrm{~s}, 3 \mathrm{H}), 3.84(\mathrm{~d}, J=14.3 \mathrm{~Hz}, 1 \mathrm{H}), 3.59(\mathrm{~d}, J=15.6 \mathrm{~Hz}$, 1H), 3.29 (d, $J=14.3 \mathrm{~Hz}, 1 \mathrm{H}), 1.22$ (s, 3H).

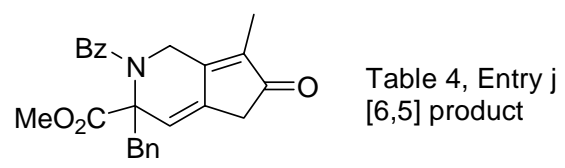

[6,5] : ${ }^{1} \mathrm{H}$ NMR $\left(300 \mathrm{MHz}, \mathrm{CDCl}_{3}\right) \delta$ 7.46-7.22 (m, $\left.10 \mathrm{H}\right), 5.77(\mathrm{~s}, 1 \mathrm{H}), 4.34(\mathrm{~d}, J=17.8$ $\mathrm{Hz}, 1 \mathrm{H}), 4.15$ (d, J = 13.8 Hz, 1H), 3.80 (s, 3H), 3.37 (d, J=13.7 Hz, 1H), 3.03 (s, 2H), $2.91(\mathrm{~d}, J=17.9 \mathrm{~Hz}, 1 \mathrm{H}), 1.45(\mathrm{~s}, 3 \mathrm{H})$. 


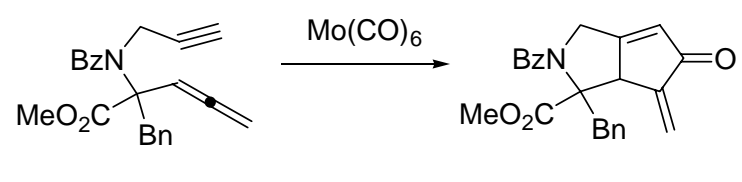

9, Table 4, Entry k

\section{2-Benzoyl-1-benzyl-6-methylene-5-oxo-1,2,3,5,6,6a-hexahydro-cyclopenta[c]pyrrole-}

1-carboxylic acid methyl ester (9, Table 4, Entry k). Followed general procedure for the molybdenum mediated allenic Pauson-Khand reaction for the formation of $[5,5] \mathrm{P}-\mathrm{K}$ products (page s38):

2-(Benzoylprop-2-ynylamino)-2-benzylhexa-3,4-dienoic acid methyl ester (435 mg, 1.21 mmol), DMSO (429 $\mu \mathrm{L}, 6.06 \mathrm{mmol}), \mathrm{Mo}(\mathrm{CO})_{6}(400 \mathrm{mg}, 1.51 \mathrm{mmol})$. Heated to $90{ }^{\circ} \mathrm{C}$ for $20 \mathrm{~min}$. The crude mixture was purified by flash chromatography (gradient elution, hexanes-EtOAc, $19: 1$ to $2: 1, \mathrm{v} / \mathrm{v})$.

Isolated two components:

$\mathrm{Rf}=0.3(\mathrm{TLC}$, hexanes-EtOAc, $3: 1 \mathrm{v} / \mathrm{v})$ [5,5] (260 mg, 55\%).

${ }^{1} \mathrm{H}$ NMR $\left(300 \mathrm{MHz}, \mathrm{CDCl}_{3}\right) \delta: 7.52-7.27(\mathrm{~m}, 10 \mathrm{H}), 6.19(\mathrm{~s}, 1 \mathrm{H}), 6.07(\mathrm{~s}, 1 \mathrm{H}), 5.63(\mathrm{~s}$, $1 \mathrm{H}), 4.24(1 / 2 \mathrm{AB}, J=14.9 \mathrm{~Hz}, 1 \mathrm{H}), 4.23(1 / 2 \mathrm{AB}, J=14.2 \mathrm{~Hz}, 1 \mathrm{H}), 4.03$ (1/2 AB, $J=$ $15.0 \mathrm{~Hz}, 1 \mathrm{H}), 3.98(\mathrm{~s}, 1 \mathrm{H}), 3.62(\mathrm{~s}, 3 \mathrm{H}), 3.39(1 / 2 \mathrm{AB}, J=14.3 \mathrm{~Hz}, 1 \mathrm{H}) ;{ }^{13} \mathrm{C} \mathrm{NMR}(75$ $\left.\mathrm{MHz}, \mathrm{CDCl}_{3}\right) \delta: 195.2,170.9,170.2,170.1,140.6,135.7,135.5,131.1,128.6,128.5$, 127.3, 126.2, 118.0, 70.8, 52.3, 51.5, 50.9, 37.7; IR (thin film) v 3027, 1741, 1710, 1641, $1383 \mathrm{~cm}^{-1}$; MS m/z (\%) 387 (15), 328 (10), 282 (22), 105 (100); EI-HRMS calcd for $\mathrm{C}_{24} \mathrm{H}_{21} \mathrm{NO}_{4} \mathrm{~m} / \mathrm{z}\left[\mathrm{M}^{+}\right] 387.1471$ found 387.1470.

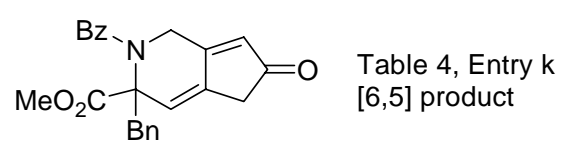

$\mathrm{Rf}=0.2(\mathrm{TLC}$, hexanes-EtOAc, $3: 1 \mathrm{v} / \mathrm{v})[6,5](79 \mathrm{mg}, 17 \%)$.

${ }^{1} \mathrm{H}$ NMR (300 MHz, $\left.\mathrm{CDCl}_{3}\right) \delta$ : 7.49-7.41 (m, 3H), 7.34-7.27 (m, 5H), 7.21-7.17 (m, 2H), 5.90 (s, 1H), $5.70(\mathrm{~s}, 1 \mathrm{H}), 4.39(1 / 2 \mathrm{AB}, J=18.1 \mathrm{~Hz}, 1 \mathrm{H}), 4.15(1 / 2 \mathrm{AB}, J=13.8 \mathrm{~Hz}$, $1 \mathrm{H}), 3.81(\mathrm{~s}, 3 \mathrm{H}), 3.38(1 / 2 \mathrm{AB}, J=13.8 \mathrm{~Hz}, 1 \mathrm{H}), 3.05(\mathrm{~s}, 2 \mathrm{H}), 3.01(1 / 2 \mathrm{AB}, J=18.1$ $\mathrm{Hz}, 1 \mathrm{H}) ;{ }^{13} \mathrm{C} \mathrm{NMR}\left(75 \mathrm{MHz}, \mathrm{CDCl}_{3}\right) \delta: 202.9,171.3,171.1,161.1,135.8,135.4,135.2$, $130.3,129.9,128.7,128.3,127.4,127.2,126.3,123.5,65.9,53.0,45.1,39.6,37.7$; IR (thin film) $v$ 2950, 1740, 1710, 1643, $1391 \mathrm{~cm}^{-1}$; MS m/z (\%) 387 (47), 356 (15), 328 (12), 296 (30), 105 (100); EI-HRMS calcd for $\mathrm{C}_{24} \mathrm{H}_{21} \mathrm{NO}_{4} \mathrm{~m} / \mathrm{z}\left[\mathrm{M}^{+}\right] 387.1471$ found 387.1489 . 


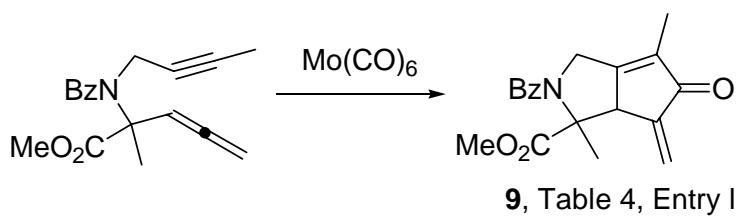

\section{2-Benzoyl-1,4-dimethyl-6-methylene-5-oxo-1,2,3,5,6,6a-hexahydrocyclopenta[c]} pyrrole-1-carboxylic acid methyl ester (9, Table 4, Entry l). Followed general procedure for the molybdenum mediated allenic Pauson-Khand reaction for the formation of $[5,5] \mathrm{P}-\mathrm{K}$ products (page s38):

2-(Benzoylbut-2-ynylamino)-2-methylpenta-3,4-dienoic acid methyl ester (50 mg, 0.17 mmol), DMSO (120 $\mu \mathrm{L}, 1.68 \mathrm{mmol}), \mathrm{Mo}(\mathrm{CO})_{6}(55 \mathrm{mg}, 0.21 \mathrm{mmol})$. Heated to $80{ }^{\circ} \mathrm{C}$ for 2 hours. After the initial filtration the crude mixture $(53 \mathrm{mg},>95 \%)$ was purified by flash chromatography (gradient elution, hexanes-EtOAc, $9: 1$ to $2: 1, \mathrm{v} / \mathrm{v}$ ) to afford the major isomer (41 mg, $74 \%)$.

Product ratios determined by ${ }^{1} \mathrm{H}$ NMR after the initial filtration: $[5,5]:[6,5]=14: 1$; Diastereomer ratio of $[5,5]$ product : $10: 1$.

$\mathrm{Rf}=0.23(\mathrm{TLC}$, hexanes-EtOAc, $2: 1, \mathrm{v} / \mathrm{v}){ }^{1} \mathrm{H}$ NMR $\left(300 \mathrm{MHz}, \mathrm{CDCl}_{3}\right) \delta: 7.54-7.44$ (m, 5H), 6.26 (d, J=1.9 Hz, 1H), $5.44(\mathrm{~s}, 1 \mathrm{H}), 4.58(1 / 2 \mathrm{AB}, J=15.7 \mathrm{~Hz}, 1 \mathrm{H}), 4.22(1 / 2$ $\mathrm{AB}, J=15.7 \mathrm{~Hz}, 1 \mathrm{H}), 4.02(\mathrm{~s}, 1 \mathrm{H}), 3.88(\mathrm{~s}, 3 \mathrm{H}), 1.82(\mathrm{~s}, 3 \mathrm{H}), 1.31(\mathrm{~s}, 3 \mathrm{H}) ;{ }^{13} \mathrm{C} \mathrm{NMR}(75$ $\left.\mathrm{MHz} \mathrm{CDCl}_{3}\right) \delta: 195.7,172.6,169.3,162.4,139.6,136.0,136.0,130.2,128.6,126.5$, 119.2, 66.5, 53.3, 52.9, 47.8, 14.6, 9.0; IR (thin film) $v 2950,1740,1707,1689,1642$, $1404 \mathrm{~cm}^{-1}$; MS m/z (\%) 325 (30), 266 (28), 220 (15), 105 (100); EI-HRMS calcd for $\mathrm{C}_{19} \mathrm{H}_{19} \mathrm{NO}_{4} \mathrm{~m} / \mathrm{z}\left[\mathrm{M}^{+}\right]$325.1314; found 325.1305.

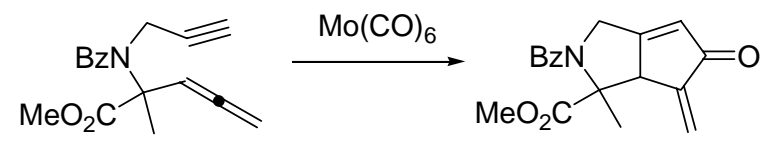

2-Benzoyl-1-methyl-6-methylene-5-oxo-1,2,3,5,6,6a-hexahydrocyclopenta[c]pyrrole 1-carboxylic acid methyl ester (9, Table 4, Entry $\mathrm{m})$. Followed general procedure for the molybdenum mediated allenic Pauson-Khand reaction for the formation of $[5,5] \mathrm{P}-\mathrm{K}$ products (page s38):

2-(Benzoylprop-2-ynylamino)-2-methylpenta-3,4-dienoic acid methyl ester (72 mg, 0.25 mmol), DMSO (180 $\mu \mathrm{L}, 2.54 \mathrm{mmol}), \mathrm{Mo}(\mathrm{CO})_{6}(84 \mathrm{mg}, 0.32 \mathrm{mmol})$. Heated to $80{ }^{\circ} \mathrm{C}$ for 2 hours. After the initial filtration the crude mixture $(80 \mathrm{mg},>95 \%)$ was purified by flash 
chromatography (gradient elution, hexanes-EtOAc, $7: 1$ to $2: 1$, v/v) to afford the major isomer (49 mg, 62\%).

Product ratios determined by ${ }^{1} \mathrm{H}$ NMR after the initial filtration: $[5,5]:[6,5]=14: 1$; Diastereomer ratio of $[5,5]$ product $: 11: 1$.

$\mathrm{Rf}=0.15\left(\mathrm{TLC}\right.$, hexanes-EtOAc, $2: 1$, v/v) ${ }^{1} \mathrm{H}$ NMR $\left(300 \mathrm{MHz}, \mathrm{CDCl}_{3}\right) \delta: 7.51-7.41$ (m, 5H), 6.26-6.25 (m, 2H), $5.46(\mathrm{~s}, 1 \mathrm{H}), 4.66(1 / 2 \mathrm{AB}, J=16.0 \mathrm{~Hz}, 1 \mathrm{H}), 4.29(1 / 2 \mathrm{AB}, J$ $=16.0 \mathrm{~Hz}, 1 \mathrm{H}), 4.13(\mathrm{~s}, 1 \mathrm{H}), 3.88(\mathrm{~s}, 3 \mathrm{H}), 1.35(\mathrm{~s}, 3 \mathrm{H}) ;{ }^{13} \mathrm{C} \mathrm{NMR}\left(75 \mathrm{MHz}, \mathrm{CDCl}_{3}\right) \delta$ : 195.6, 172.8, 170.4, 169.6, 140.2, 136.2, 130.6, 129.0, 127.9, 126.8, 120.0, 66.8, 55.4, 53.3, 49.2, 17.3, 15.0; IR (thin film) v 2950, 1739, 1709, 1638, $1402 \mathrm{~cm}^{-1}$; MS m/z (\%) 311 (18), 252 (22), 206 (9), 105 (100); EI-HRMS calcd for $\mathrm{C}_{18} \mathrm{H}_{17} \mathrm{NO}_{4} \mathrm{~m} / \mathrm{z}\left[\mathrm{M}^{+}\right]$ 311.1158 ; found 311.1151 .

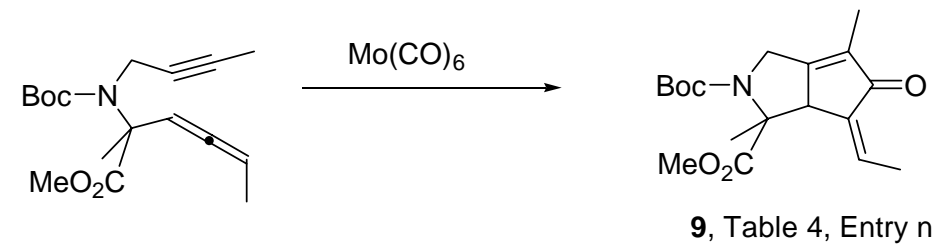

\section{6-Ethylidene-1,4-dimethyl-5-oxo-3,5,6,6a-tetrahydro-1H-cyclopenta[c]pyrrole-1,2-} dicarboxylic acid 2-tert-butyl ester 1-methyl ester (9, Table 4, Entry n). Followed general procedure for the molybdenum mediated allenic Pauson-Khand reaction for the formation of [5,5] P-K products (page s38):

2-(tert-Butoxycarbonyl-but-2-ynylamino)-2-methylhexa-3,4-dienoic acid methyl ester (45 mg, $0.15 \mathrm{mmol}$ ), DMSO (106 $\mu \mathrm{L}, 1.49 \mathrm{mmol}), \mathrm{Mo}(\mathrm{CO})_{6}(49 \mathrm{mg}, 0.19 \mathrm{mmol})$. Heated to $85{ }^{\circ} \mathrm{C}$ for 2.5 hours. After the initial filtration the crude mixture ( $45 \mathrm{mg},>90 \%$ ) was purified by flash chromatography (gradient elution, hexanes-EtOAc, $9: 1$ to $4: 1$, v/v) to afford partial separation of the major from the minor isomer $\mathrm{Z} / \mathrm{E}=5: 1$ (NMR).

(Z) : Rf $=0.5($ TLC, hexanes-EtOAc, $4: 1, \mathrm{v} / \mathrm{v}){ }^{1} \mathrm{H}$ NMR $\left(300 \mathrm{MHz}\right.$, DMSO- $\left.d_{6} 353 \mathrm{~K}\right) \delta$ 5.99-5.91 (m, 1H), $4.31(1 / 2 \mathrm{AB}, J=16.7 \mathrm{~Hz}, 1 \mathrm{H}), 4.23(1 / 2 \mathrm{AB}, J=16.1 \mathrm{~Hz}, 1 \mathrm{H}), 3.84$ (br s, 1H), 3.75 (s, 3H), 2.18 (d, J =6.8 Hz, 3H), 1.75 (s, 3H), 1.39 (s, 9H), 1.04 (s, 3H); IR (thin film) $v$ 2977, 1744, 1696, 1647, $1390 \mathrm{~cm}^{-1}$; MS m/z (\%) 335 (19), 279 (10), 235 (60), 220 (38), 57 (100) ; EI-HRMS calcd for $\mathrm{C}_{18} \mathrm{H}_{25} \mathrm{NO}_{5} \mathrm{~m} / \mathrm{z}\left[\mathrm{M}^{+}\right]$335.1733; found 335.1748 . 
N-Benzamide protected alkynyl allenes 6 (Table 1, Entry f, g and o) reacted in the $\mathrm{Mo}(\mathrm{CO})_{6}$ mediated $\mathrm{P}-\mathrm{K}$ reaction to afford products as complex mixture of diastereomers and $\mathrm{Z} / \mathrm{E}$ isomers. Due to difficulties in purification no spectral data is available. The yields of crude mixtures are reported.
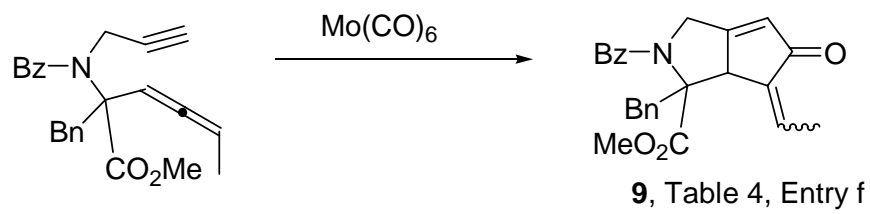

\section{2-Benzoyl-1-benzyl-6-ethylidene-4-methyl-5-oxo-1,2,3,5,6,6a-hexahydro-} cyclopenta[c]pyrrole-1-carboxylic acid methyl ester (9, Table 4, Entry f). Followed general procedure for the molybdenum mediated allenic Pauson-Khand reaction for the formation of $[5,5] \mathrm{P}-\mathrm{K}$ products (page s38):

2-(Benzoylprop-2-ynylamino)-2-benzylhexa-3,4-dienoic acid methyl ester (42 mg, 0.11 mmol), DMSO (75 $\mu \mathrm{L}, 1.1 \mathrm{mmol}), \mathrm{Mo}(\mathrm{CO})_{6}(37 \mathrm{mg}, 0.14 \mathrm{mmol})$. Heated at $75{ }^{\circ} \mathrm{C}$ for 4 h. Weight of mixture after flash chromatography (26 mg, 57\%). The starting material was recovered in $25 \%$.
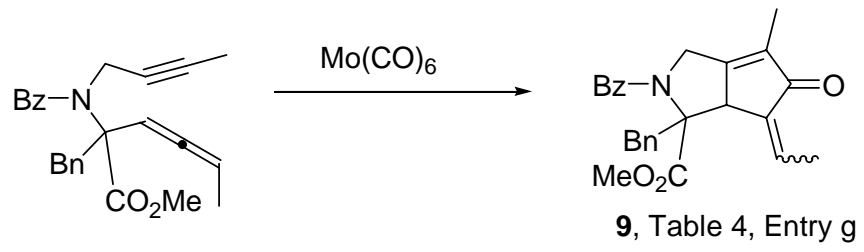

\section{2-Benzoyl-1-benzyl-6-ethylidene-4-methyl-5-oxo-1,2,3,5,6,6a-hexahydro-} cyclopenta[c]pyrrole-1-carboxylic acid methyl ester (9, Table 4, Entry g). Followed general procedure for the molybdenum mediated allenic Pauson-Khand reaction for the formation of [5,5] P-K products (page s38):

2-(benzoyl-but-2-ynyl-amino)-2-benzylhexa-3,4-dienoic acid methyl ester (36 mg, 0.093 $\mathrm{mmol}), \operatorname{DMSO}(62 \mu \mathrm{L}, 0.93 \mathrm{mmol}), \mathrm{Mo}(\mathrm{CO})_{6}(31 \mathrm{mg}, 0.12 \mathrm{mmol})$. Heated to $80{ }^{\circ} \mathrm{C}$ for 1 h. The reaction mixture was then cooled to room temperature, and applied to a silica gel column. Elution (hexanes-EtOAc, $2: 1$, v/v) afforded a mixture of compounds (29 mg, $77 \%$ ). 


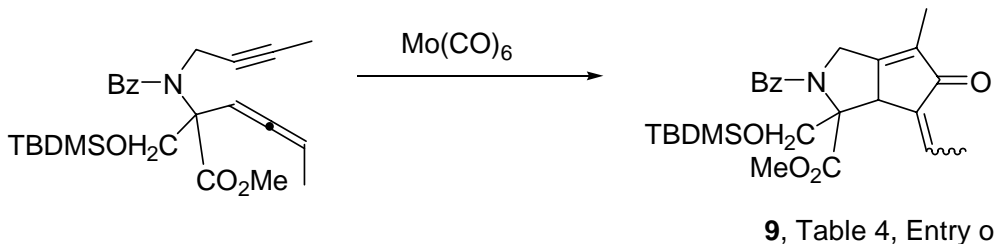

2-Benzoyl-1-(tert-butyldimethylsilyloxymethyl)-6-ethylidene-4-methyl-5-oxo1,2,3,5,6,6a-hexahydro-cyclopenta[c]pyrrole-1-carboxylic acid methyl ester (9, Table 4, Entry o). Followed general procedure for the molybdenum mediated allenic PausonKhand reaction for the formation of [5,5] P-K products (page s38):

2-(Benzoylbut-2-ynylamino)-2-(tert-butyldimethylsilyloxymethyl)-hexa-3,4-dienoic acid methyl ester (44 mg, $0.10 \mathrm{mmol})$, DMSO (70 $\mu \mathrm{L}, 1.0 \mathrm{mmol}), \mathrm{Mo}(\mathrm{CO})_{6}(33 \mathrm{mg}, 0.12$ mmol). Heated to $85{ }^{\circ} \mathrm{C}$ for $1 \mathrm{~h}$. Weight of mixture after flash chromatography $(50 \mathrm{mg}$, $>95 \%)$. 